\title{
Efficient Parallel Sorting for Migrating Birds Optimization When Solving Machine-Part Cell Formation Problems
}

\author{
Ricardo Soto, ${ }^{1,2,3}$ Broderick Crawford, ${ }^{1,4,5}$ Boris Almonacid, ${ }^{1}$ and Fernando Paredes ${ }^{6}$ \\ ${ }^{1}$ Pontificia Universidad Católica de Valparaíso, 2362807 Valparaíso, Chile \\ ${ }^{2}$ Universidad Autónoma de Chile, 7500138 Santiago, Chile \\ ${ }^{3}$ Universidad Científica del Sur, Lima 18, Peru \\ ${ }^{4}$ Universidad Central de Chile, 8370178 Santiago, Chile \\ ${ }^{5}$ Universidad San Sebastián, 8420524 Santiago, Chile \\ ${ }^{6}$ Universidad Diego Portales, 8370109 Santiago, Chile \\ Correspondence should be addressed to Boris Almonacid; boris.almonacid.g@mail.pucv.cl
}

Received 15 January 2016; Accepted 25 February 2016

Academic Editor: Eduardo Rodríguez-Tello

Copyright (C) 2016 Ricardo Soto et al. This is an open access article distributed under the Creative Commons Attribution License, which permits unrestricted use, distribution, and reproduction in any medium, provided the original work is properly cited.

\begin{abstract}
The Machine-Part Cell Formation Problem (MPCFP) is a NP-Hard optimization problem that consists in grouping machines and parts in a set of cells, so that each cell can operate independently and the intercell movements are minimized. This problem has largely been tackled in the literature by using different techniques ranging from classic methods such as linear programming to more modern nature-inspired metaheuristics. In this paper, we present an efficient parallel version of the Migrating Birds Optimization metaheuristic for solving the MPCFP. Migrating Birds Optimization is a population metaheuristic based on the V-Flight formation of the migrating birds, which is proven to be an effective formation in energy saving. This approach is enhanced by the smart incorporation of parallel procedures that notably improve performance of the several sorting processes performed by the metaheuristic. We perform computational experiments on 1080 benchmarks resulting from the combination of 90 well-known MPCFP instances with 12 sorting configurations with and without threads. We illustrate promising results where the proposal is able to reach the global optimum in all instances, while the solving time with respect to a nonparallel approach is notably reduced.
\end{abstract}

\section{Introduction}

The Machine-Part Cell Formation Problem (MPCFP) is based on the well-known Group Technology (GP) [1] widely used in the manufacturing industry. The goal of the MPCFP is to organize a manufacturing plant in a set of cells that contain a limited number of machines and parts, but minimize the cell-interchange of parts. The purpose is to reduce costs and increase productivity. The MPCFP is known to be NPHard, so there is always the challenge of producing high quality solutions in a limited time interval. During the last years, several techniques have successfully been applied to this problem, from mathematical methods such as linear programming [2] to classic metaheuristics such as particle swarm optimization [3] and more modern ones such as artificial fish swarms [4].

In this paper, we present a new and efficient parallel version of the Migrating Birds Optimization metaheuristic for solving the MPCFP. Migrating Birds Optimization (MBO) is a population-based metaheuristic inspired by the $\mathrm{V}$-shaped flight employed by birds when they migrate. This technique is known to be very effective for energy saving during flying. This interesting approach is enhanced by precisely integrating parallel procedures particularly for the efficient sorting of birds and neighboring solutions. It results in a notable improvement in terms of performance of the whole solving process. We perform computational experiments by using 90 well-known MPCFP instances, 2 well-known sorting algorithms, 1 sequential configuration, and 5 thread configurations $(1,4,8,16$, and 32) resulting in a total of 1080 benchmarks. The obtained results are encouraging where the proposal is able to reach the global optimum in all instances, while the solving time with respect to a nonparallel approach is notably reduced.

The outline of the study is as follows. In Section 2, we give some information on the related work. Section 3 describes 
TABLE 1: Initial incidence matrix $M \times P$.

\begin{tabular}{lccccccc}
\hline & Part 1 & Part 2 & Part 3 & Part 4 & Part 5 & Part 6 & Part 7 \\
\hline Machine 1 & 0 & 1 & 0 & 1 & 0 & 0 \\
Machine 2 & 1 & 0 & 1 & 0 & 0 & 0 & 0 \\
Machine 3 & 1 & 0 & 0 & 1 & 0 & 0 \\
Machine 4 & 0 & 1 & 0 & 0 & 0 & 1 \\
Machine 5 & 1 & 0 & & & 0 & 0 \\
\hline
\end{tabular}

and models the MPCFP. Section 4 gives an overview of MBO. Section 5 describes how to resolve the MPCFP using MBO with parallel sort. Section 6 presents and discusses the experimental results. Section 7 concludes and provides guidelines for future work. Finally, the Appendix provides detailed results for the 1080 benchmarks, in which each includes 90 test instances.

\section{Related Work}

Several investigations that have been carried out for the problem MPCFP are as follows: linear programming [5], a goal programming model [6], a hybrid genetic algorithm [7], boolean satisfiability [8], constraint programming [9], and using an artificial fish swarm algorithm [4]. An analysis of the evolution of cell formation problem can be found in $[10,11]$. As MPCFP is NP-Hard problem, many researchers have considered applying approximation methods as metaheuristics. Various research can be found in the field of metaheuristics,.in which the search is performed using iterative procedures, which allow moving from one solution to another in the search space. This type of metaheuristic performs movements in the neighborhood of the current solution, which means it has a perturbative nature. As metaheuristics are based on trajectories, among which we have simulated annealing [12-14], tabu search [15-17], local iterated search $[18,19]$, and local variable search [20], there is also another type of metaheuristics which are based on population. The population metaheuristics have a set of individuals, where each individual encodes a temporary solution. These metaheuristics use an evaluation function fitness of each individual, with the aim of obtaining the best values of the population that solve an optimization problem. In addition, there are disturbances in the algorithm that directed individuals in the population or part of them to possible solutions for better fitness. Among these population-based metaheuristics, ant colony optimization [21, 22], particle swarm optimization $[3,23,24]$, cat swarm $[25,26]$, and Migrating Birds Optimization [27] can be found in problems like a hybrid flowshop scheduling with total flowtime minimisation [28], closed loop layout with exact distances in flexible manufacturing systems [29], and preliminary results for MPCFP [30]. A brief review of nature-inspired algorithms for optimization can be found in [31].

In the area of manufacturing and industrial applications, parallel implementations using metaheuristics have been addressed, such as GRASP and grid computing to solve the location area problem [32], performance analysis of coarse-grained parallel genetic algorithms on the multicore
Sun Ultra SPARC T1 [33], a parallel genetic algorithm for the multilevel unconstrained lot-sizing problem [34], and a memetic algorithm and a parallel hyperheuristic islandbased model for a 2D packing problem [35]. We can also find research using parallel metaheuristics as a cooperative parallel metaheuristic for the capacitated vehicle routing problem [36] and optimizing shared-memory hyperheuristics on top of parameterized metaheuristics [37] and, finally, we can find a review of recent progress in implementing parallelism with metaheuristics included in the following work [38].

Different studies have addressed the parallel sorting algorithms as massively parallel sort-merge joins in main memory multicore database systems [39], an efficient parallel merge sort for fixed and variable length keys [40], a randomized parallel sorting algorithm with an experimental study [41], sorting algorithm for many-core architectures based on adaptive bitonic sort [42], performance comparison of sequential quick sort and parallel quick sort algorithms [43], the time profit obtained by parallelization of quick sort algorithm used for numerical sorting [44], an efficient massively parallel quick sort [45], and, finally, a fast parallel implementation of quick sort and its performance evaluation on SUN enterprise 10000 [46].

In this paper, we have chosen metaheuristic MBO because it has a friendly design, composed of two linked lists for each of the sides of the flock of birds and a node as a leader bird. Furthermore, MBO has been applied to various optimization problems with promising results [47-49]. We focus on an efficient parallel sorting for MBO when solving MPCFP, which to our knowledge has not yet been reported. It has been chosen to parallelize the sorting algorithms in MBO because 4 out of the 8 steps of the MBO metaheuristic used sorting algorithms, and therefore we believe that applying parallel sorting in these steps is possible to achieve reduction of the execution time in $\mathrm{MBO}$ algorithm.

\section{Machine-Part Cell Formation Problems}

The Machine-Part Cell Formation Problem (MPCFP) is NP-Hard optimization problem; the main objective is the formation of set of machines and parts in groups so that the number of intercell transportation of parts is minimized. Therefore, the initial matrix (see matrix in Table 1) must be converted into a matrix that has a block diagonal structure (cells are easily visible; see matrix in Table 2). This example corresponds to a MPCFP with the following parameters: 5 machines, 7 parts, and $M_{\max }=3$ for 2 cells. The optimum value obtained is 0 , and the final incidence matrix $a_{i j}$ is 
TABLE 2: Rearranged incidence matrix $M \times P$.

\begin{tabular}{|c|c|c|c|c|c|c|c|}
\hline & \multicolumn{3}{|c|}{ Cell 1} & \multicolumn{4}{|c|}{ Cell 2} \\
\hline & Part 1 & Part 3 & Part 7 & Part 2 & Part 4 & Part 6 & Part 5 \\
\hline \multicolumn{8}{|l|}{ Cell 1} \\
\hline Machine 2 & 1 & 1 & 0 & 0 & 0 & 0 & 0 \\
\hline Machine 3 & 1 & 1 & 1 & 0 & 0 & 0 & 0 \\
\hline Machine 5 & 1 & 0 & 1 & 0 & 0 & 0 & 0 \\
\hline \multicolumn{8}{|l|}{ Cell 2} \\
\hline Machine 1 & 0 & 0 & 0 & 1 & 1 & 1 & 1 \\
\hline Machine 4 & 0 & 0 & 0 & 1 & 1 & 1 & 0 \\
\hline
\end{tabular}

TABLe 3: Matrix $M \times C \rightarrow y_{i k}$.

\begin{tabular}{lcc}
\hline & Cell 1 & Cell 2 \\
\hline Machine 1 & 0 & 1 \\
Machine 2 & 1 & 0 \\
Machine 3 & 1 & 0 \\
Machine 4 & 0 & 1 \\
Machine 5 & 1 & 0 \\
\hline
\end{tabular}

TABle 4: Matrix $P \times C \rightarrow z_{j k}$.

\begin{tabular}{lcc}
\hline & Cell 1 & Cell 2 \\
\hline Part 1 & 1 & 0 \\
Part 2 & 0 & 1 \\
Part 3 & 1 & 0 \\
Part 4 & 0 & 1 \\
Part 5 & 0 & 1 \\
Part 6 & 0 & 1 \\
Part 7 & 1 & 0 \\
\hline
\end{tabular}

constructed from the results of the matrices $y_{i k}$ and $z_{j k}$ (see Tables 3 and 4).

A rigorous mathematical formulation of MPCFP is given by Boctor [2]. The problem is represented by the following mathematical model:

(i) Parameters, index, and sets:

(a) $a_{i j}$, the $M \times P$ binary machine-part incidence matrix, where

$a_{i j}= \begin{cases}1 & \text { if } j_{\text {th }} \text { part visits } i_{\text {th }} \text { machine; } \\ 0 & \text { otherwise, }\end{cases}$

(b) $M$, the number of machines,

(c) $P$, the number of parts,

(d) $C$, the number of cells,

(e) $i$, the index of machines $(i=1, \ldots, M)$,

(f) $j$, the index of parts $(j=1, \ldots, P)$,

(g) $k$, the index of cells $(k=1, \ldots, C)$,

(h) $M_{\max }$, the maximum number of machines per cell. (ii) Variables and domains:

(a) $y_{i k}$, the $M \times C$ machine-cell matrix, where

$$
y_{i k}= \begin{cases}1 & \text { if machine } i \in \text { cell } k \\ 0 & \text { otherwise, }\end{cases}
$$

(b) $z_{j k}$, the $P \times C$ part-cell matrix, where

$$
z_{j k}= \begin{cases}1 & \text { if part } j \in \text { family } k \\ 0 & \text { otherwise. }\end{cases}
$$

(iii) Objective function:

$$
\text { minimized } \sum_{k=1}^{C} \sum_{i=1}^{M} \sum_{j=1}^{P} a_{i j} z_{j k}\left(1-y_{i k}\right)
$$

(iv) Being subject to the following constraints:

$$
\begin{aligned}
& \sum_{k=1}^{C} y_{i k}=1 \quad \forall_{i}, \\
& \sum_{i=1}^{M} y_{i k} \leq M_{\max } \quad \forall_{k}, \\
& \sum_{k=1}^{C} z_{j k}=1 \quad \forall_{j} .
\end{aligned}
$$

\section{Migrating Birds Optimization}

Migrating Birds Optimization (MBO) is a nature-inspired population metaheuristic based on the $\mathrm{V}$-shaped flight of the migrating birds [50,51], which is proven to be an effective formation in energy saving [52]. In the V-formation (shown in Figure 1), some parameters like wing-tip spacing (WTS), maximum width of the wing $(w)$, angle of the $\mathrm{V}$-formation $(\alpha)$, and depth and wing span (b) are important to form an effective V-formation [53,54]. The conceptual similarity between the parameters of the algorithm of $\mathrm{MBO}$ with the actual migration of birds in V-flight formation is investigated in Duman et al. [27]. This conceptual similarity is described in Table 5. 
TABLE 5: Conceptual similarity of MBO metaheuristic and V-shape natural migration of birds.

\begin{tabular}{ll}
\hline Parameter description & Concept in real migration birds in V-formation \\
\hline $\begin{array}{l}\text { The number of initial solutions of the flock. } \\
\text { The number of neighboring solutions generated for each initial } \\
\text { solution. }\end{array}$ & $\begin{array}{l}\text { Birds in V-formation. } \\
\text { The induced power required which is inversely proportional to the } \\
\text { speed. }\end{array}$ \\
$\begin{array}{l}\text { The number of neighboring solutions shared with the next solution. } \\
\begin{array}{l}\text { The number of tours. } \\
\text { The number of iterations (total number of generated neighbor } \\
\text { solutions). }\end{array}\end{array}$ & $\begin{array}{l}\text { The number of wing flaps before a change occurs in the leading bird. } \\
\text { There is no conceptual relationship. }\end{array}$ \\
\hline
\end{tabular}

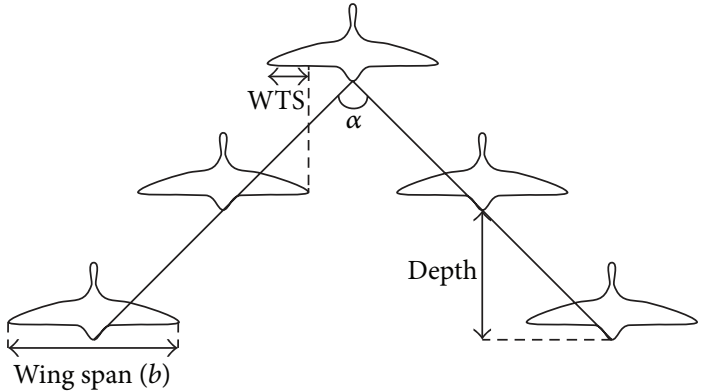

FIGURE 1: The V-shaped flight.

The MBO starts with a number of $n$ initial solutions corresponding to birds in a $\mathrm{V}$-flight formation. These solutions are generated randomly and a solution as the leader bird is chosen; then the remaining birds are alternately distributed to each side of the flock. In the next step, for all tours, improving the leader solution is performed by generating and evaluating its $k$ neighbors. For each solution $S_{r}$ in the flock (except leader), try to improve $S_{r}$ by evaluating its $(k-x)$ neighbors and $x$ unused best neighbors from the solution in the front. Move the leader solution to the end and forward one of the solutions following it to the leader position. Finally, when the iterations are over, return the best solution in the flock. Algorithm 1 depicts the classic procedure of $\mathrm{MBO}$ algorithm.

\section{Solving the MPCFP Using MBO with Parallel Sort}

The first step in solving the problem MPCFP is a proper integration with the $\mathrm{MBO}$ metaheuristic. In this sense, we conceptualize every bird of the flock (including the leader of the flock) as a possible solution to the problem MPCFP (see Figure 2). Each bird will be composed of a matrix $M \times C$ and matrix $P \times C$. These matrices are those modified while the problem lapsed iterations. In addition, they will be used for the calculation of fitness to problem MPCFP.

5.1. Generate Initial Solutions. For generating initial solutions, consider the following inputs: machines $=4$, parts $=$ 5 , cells $=2, M_{\max }=2$, and a matrix $M \times P$ (see Table 6).

Considering data in Table 6, the first step to generate an initial solution is as follows.
Step 1. Generate random allocations in the matrix $M \times P$ with some random method. This matrix $M \times C$ (see Table 7) satisfies constraints that exists in only one machine in a cell and $M_{\max }$ is less than or equal to 2 in an entire cell.

Step 2. Generate with a manual method the matrix $P \times C$ from the matrix $M \times C$. For this, a connection is established with the matrix $M \times P$ and with the matrix solution $M \times C$. In lightface, machines correspond to the cell number 1 and, in bold, machines correspond to cell number 2 (see Table 8 ).

The manual method for determining the matrix $P \times C$ involves adding the value 1 out of every position $i, j$ of the $M \times P$ matrix and putting this value in the temporary array of sums according to the corresponding cell. We perform the operation for each value of positions $i, j$ of the matrix $M \times P$ (see Table 9).

\section{Example 1.}

(i) $M \times P[1,1]=1 \rightarrow$ is the value that is associated with cell 1; we add this value to the Row Sum of Cell 1.

(ii) $M \times P[2,1]=1 \rightarrow$ is the value that is associated with cell 2; we add to this value to the Row Sum of Cell 2.

(iii) $M \times P[3,1]=0 \rightarrow$ is the value that is associated with cell 2; we add to this value to the Row Sum of Cell 2.

(iv) $M \times P[4,1]=1 \rightarrow$ is the value that is associated with cell 1; we add to this value to the Row Sum of Cell 1.

Step 3. From the results of Table 9, we build a partial solution to the matrix $P \times C$ (see Table 10).

Step 4. Find possible solutions to $P \times C$.

We must choose the values of the positions $i, j$ that belong to the temporary matrix $M \times P$ and have the largest values of each row. Subsequently, we replace larger values assigning them the value 1 , and for the other values in the row we assign them the value of 0 (see Table 11). In case of draw, that is, the entire row has equal numbers, we keep unchanged if the value of their positions is 1 . In the case that the same numbers are greater than 1, to each number greater than 1, we assign them a value of 1 .

We consider rows having values equal to 1 in this case correspond to rows pertaining to machines 3 and 4 . From this point, we generate all possible combinations of matrices and randomly choose one (see Table 12). 
(1) Generate $n$ initial solutions in V-formation

(2) $i=0$

(3) while $(i<I)$ do

(4) for int $j=0 ; j<m ; j++$ do

(5) Improve the leading solution by generating and evaluating $k$ neighbors of it

(6) $\quad i=i+k$

(7) for each solution $S_{r}$ in the flock (except leader) do

(8) Try to improve $S_{r}$ by evaluating $(k-x)$ neighbors of it and $x$ unused best neighbors from the leader

(9) $\quad i=i+(k-x)$

(10) end

(11) end

(12) Move the leader solution to the end and forward one of the solutions following it to the leader position

(13) end

(14) Return the best solution in the flock

Algorithm 1: Pseudocode of Migrating Birds Optimization.

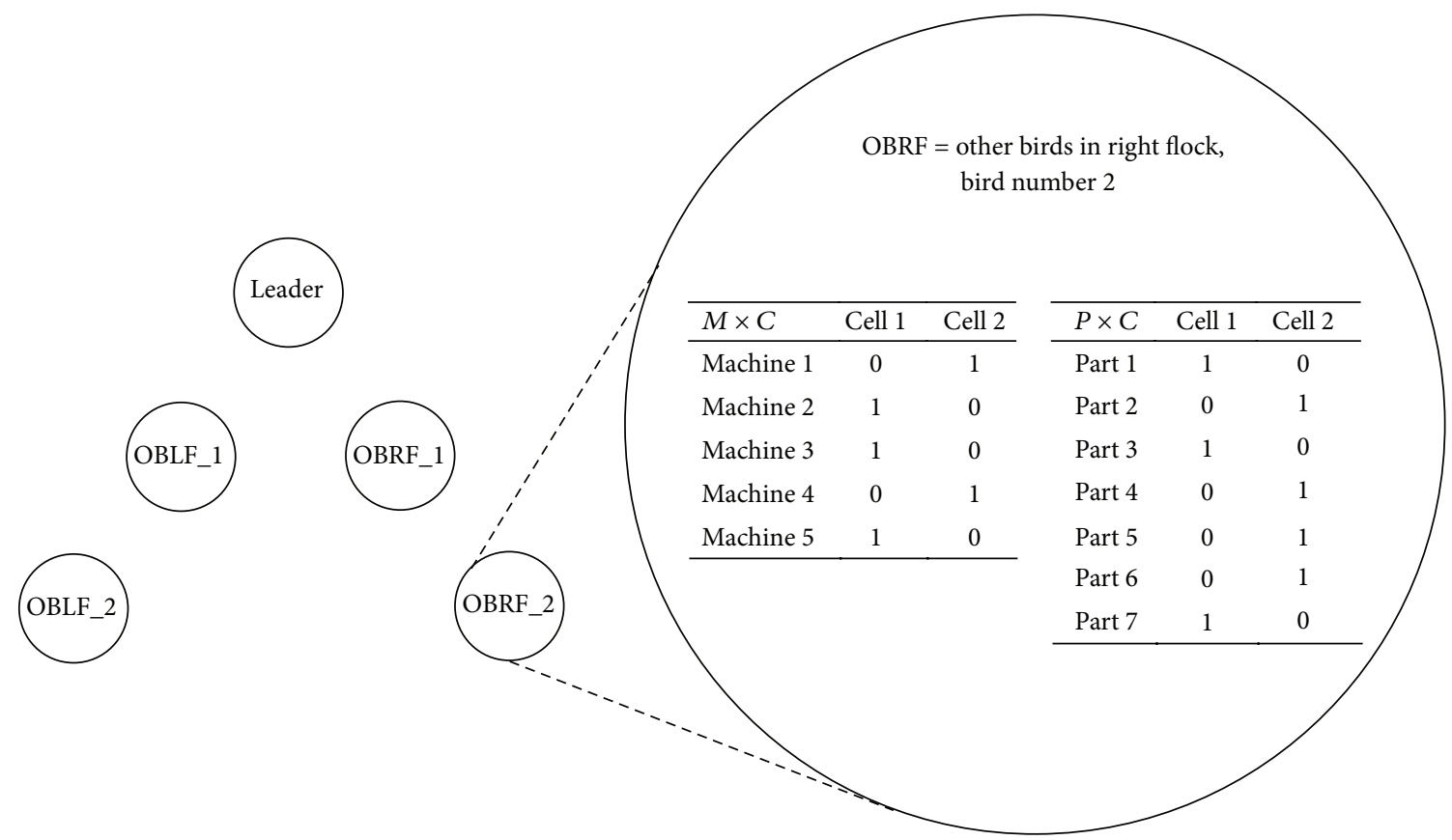

Figure 2: Integration of Migrating Birds Optimization with the Machine-Part Cell Formation Problem.

TABLE 6: Matrix $M \times P$.

\begin{tabular}{lccccc}
\hline & Part 1 & Part 2 & Part 3 & Part 4 & Part 5 \\
\hline Machine 1 & 1 & 0 & 0 & 1 & 1 \\
Machine 2 & 1 & 0 & 1 & 0 & 1 \\
Machine 3 & 0 & 1 & 0 & 1 & 1 \\
Machine 4 & 1 & 0 & 1 & 0 & 0 \\
\hline
\end{tabular}

5.2. Generating Neighboring Solutions. To generate a neighboring solution, we consider the following inputs: machines $=4$, parts $=5$, cells $=2, M_{\max }=2$, matrix $M \times P$, matrix $M \times C$, and matrix $P \times C$ (see Table 13).
TABLE 7: Matrix $M \times C$.

\begin{tabular}{lcc}
\hline & Cell 1 & Cell 2 \\
\hline Machine 1 & 1 & 0 \\
Machine 2 & 0 & 1 \\
Machine 3 & 0 & 1 \\
Machine 4 & 1 & 0 \\
\hline
\end{tabular}

Step 1. Randomly choose a position $i, j$ in the matrix $M \times C$ that has a value of 1 and we work with the machine associated with that row $i$. This is the same as selecting a machine and working with its row $i$. In this case, we chose randomly the 
TABLE 8: Relationship between the matrix $M \times P$ with the matrix $M \times C$.

\begin{tabular}{lccccccc}
\hline & Part 1 & Part 2 & Part 3 & Part 4 & Part 5 & Cell 1 & Cell 2 \\
\hline Machine 1 & 1 & 0 & 0 & 1 & $\mathbf{0}$ & 1 & 0 \\
Machine 2 & $\mathbf{1}$ & $\mathbf{0}$ & $\mathbf{1}$ & $\mathbf{1}$ & $\mathbf{0}$ & $\mathbf{1}$ \\
Machine 3 & $\mathbf{0}$ & $\mathbf{1}$ & 1 & $\mathbf{1}$ & $\mathbf{0}$ & $\mathbf{1}$ \\
Machine 4 & 1 & 0 & 0 & 0 & 0 \\
\hline
\end{tabular}

TABLE 9: Generation of temporary matrix sum.

\begin{tabular}{|c|c|c|c|c|c|c|c|}
\hline & Part 1 & Part 2 & Part 3 & Part 4 & Part 5 & Cell 1 & Cell 2 \\
\hline Machine 1 & 1 & 0 & 0 & 1 & 1 & 1 & 0 \\
\hline Machine 2 & 1 & $\mathbf{0}$ & 1 & $\mathbf{0}$ & 1 & $\mathbf{0}$ & 1 \\
\hline Machine 3 & $\mathbf{0}$ & 1 & $\mathbf{0}$ & 1 & 1 & $\mathbf{0}$ & 1 \\
\hline Machine 4 & 1 & 0 & 1 & 0 & 0 & 1 & 0 \\
\hline Sum of Cell 1 & 2 & 0 & 1 & 1 & 1 & & \\
\hline Sum of Cell 2 & 1 & 1 & 1 & 1 & 2 & & \\
\hline
\end{tabular}

TABle 10: Matrix $P \times C$.

\begin{tabular}{lcc}
\hline & Cell 1 & Cell 2 \\
\hline Part 1 & 2 & 1 \\
Part 2 & 0 & 1 \\
Part 3 & 1 & 1 \\
Part 4 & 1 & 1 \\
Part 5 & 1 & 2 \\
\hline
\end{tabular}

TABLE 11: Matrix $P \times C$.

\begin{tabular}{lccccc}
\hline & Cell 1 & Cell 2 & Cell 1 & Cell 2 \\
\hline Part 1 & 2 & 1 & & 1 & 0 \\
Part 2 & 0 & 1 & & 0 & 1 \\
Part 3 & 1 & 1 & $\rightarrow$ & 1 & 1 \\
Part 4 & 1 & 1 & & 1 & 1 \\
Part 5 & 1 & 2 & & 0 & 1 \\
\hline
\end{tabular}

position of the matrix $M \times C[2,2]$ with a value of 1 , which corresponds to machine 2 (see Table 14).

Step 2. Then on machine 2, we randomly pick a cell that has a 0 . In this case, we choose the position of the matrix $M \times$ $C[2,1]$; we accomplish a replacement of the value of 0 to 1 and finally realize a replacement for $M \times C[2,2]$ value of 1 to 0 . You can see the result of this operation in Table 15. In the case that the constraints are inconsistent, you must repeat the procedure from Step 1.

Step 3. We carry out the construction of the matrix $P \times C$ using the manual method explained in Section 5.1.

5.3. Flowchart of Migrating Birds Optimization. This section describes the stages of the algorithm $\mathrm{MBO}$, as it integrates with the problem of MPCFP and in which stages parallel arrangement algorithms are applied. For a better understanding, a flow chart has been included (see Figure 3) which is divided into 4 main phases: algorithm initialization, a tour process, leader replacement, and algorithm finalization.
5.3.1. Algorithm Initialization. Parameter Initialization (Stage 1 in Figure 3). At this stage, all the values required by the MBO metaheuristic are initialized; all the values required by the problem of MPCFP and that type of sorting algorithm are to be implemented in the metaheuristic.

Among the metaheuristic values, there are

(i) number of birds $(n)$,

(ii) number of neighbors $(k)$,

(iii) number of tours $(m)$,

(iv) number of shared solutions $(x)$,

(v) number of iterations (I).

And between complementary metaheuristic parameters, we can include

(i) leader exchange mode, where there are 2 types of changes of leader; exchange the leader with the successor and exchange the leader with the best;

(ii) sort type applied in the initial birds flock, where there is an ordering with random birds in the flock and another with birds ordered according to their fitness.

Among the parameters required for the problem MPCFP, we have

(i) incidence matrix $(M \times P)$,

(ii) number of machines $(M)$,

(iii) number of parts $(P)$,

(iv) number of cells $(C)$,

(v) maximum number of machines in a cell $\left(M_{\max }\right)$.

Finally, for the types of sorting applied to $\mathrm{MBO}$, we have the following parameters:

(i) Type of sorting algorithm: quick sort or merge sort.

(ii) Sorting technique: normal or with threads.

(iii) Number of threads if required. 
TABle 12: All possible combinations of matrices $P \times C$.

\begin{tabular}{lccccc}
\hline & Cell 1 & Cell 2 & & Cell 1 & Cell 2 \\
\hline Part 1 & 1 & 0 & Part 1 & 1 & 0 \\
Part 2 & 0 & 1 & Part 2 & 1 & 0 \\
Part 3 & 1 & 0 & Part 3 & 0 & 0 \\
Part 4 & 1 & 0 & Part 4 & Part 5 & 1 \\
Part 5 & 0 & 1 & & 1 & 0 \\
\hline & Cell 1 & Cell 2 & Part 1 & 0 \\
Part 1 & 1 & 0 & Part 2 & 0 & 1 \\
Part 2 & 0 & 1 & Part 3 & 0 & 1 \\
Part 3 & 0 & 1 & Part 4 & 0 \\
Part 4 & 1 & 0 & Part 5 & 1 \\
Part 5 & 0 & 1 &
\end{tabular}

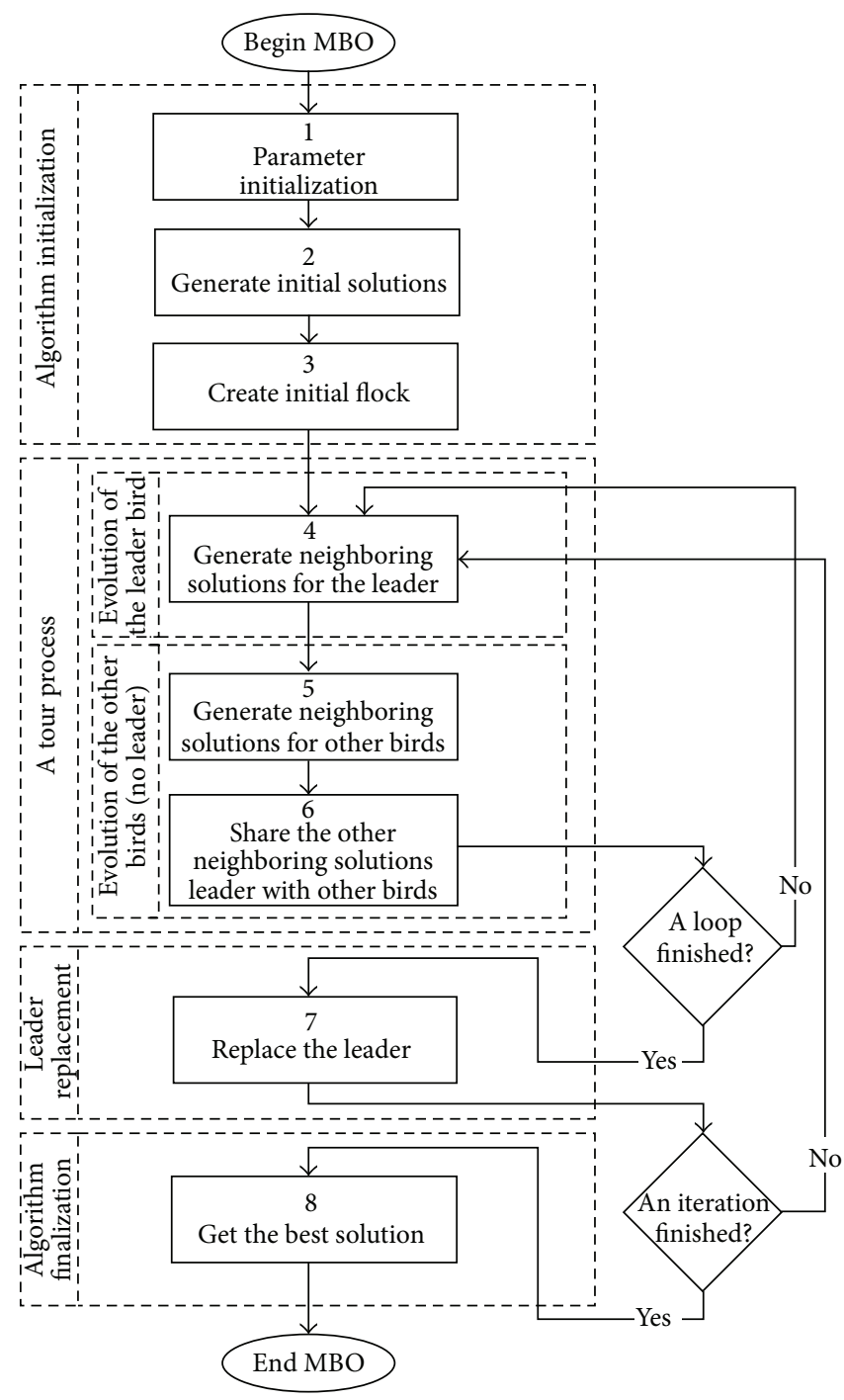

FIgURE 3: Flowchart of Migrating Birds Optimization. 
TABLE 13: Initial matrices for generating neighboring solutions.

(a)

\begin{tabular}{lccccc}
\hline & Cell 1 & Cell 2 & Cell 3 & Cell 4 & Cell 5 \\
\hline Machine 1 & 1 & 0 & 0 & 1 & 1 \\
Machine 2 & 1 & 0 & 1 & 0 & 1 \\
Machine 3 & 0 & 1 & 0 & 1 & 1 \\
Machine 4 & 1 & 0 & 1 & 0 & 0 \\
\hline
\end{tabular}

(b)

\begin{tabular}{lcc}
\hline & Cell 1 & Cell 2 \\
\hline Machine 1 & 1 & 0 \\
Machine 2 & 0 & 1 \\
Machine 3 & 0 & 1 \\
Machine 4 & 1 & 0 \\
\hline
\end{tabular}

(c)

\begin{tabular}{lcc}
\hline & Cell 1 & Cell 2 \\
\hline Part 1 & 1 & 0 \\
Part 2 & 0 & 1 \\
Part 3 & 0 & 1 \\
Part 4 & 1 & 0 \\
Part 5 & 0 & 1 \\
\hline
\end{tabular}

TABLE 14: Machine selection.

(a)

\begin{tabular}{lcc}
\hline & Cell 1 & Cell 2 \\
\hline Machine 1 & 1 & 0 \\
Machine 2 & 0 & 1 \\
Machine 3 & 0 & 1 \\
Machine 4 & 1 & 0 \\
\hline
\end{tabular}

(b)

\begin{tabular}{lcc}
\hline & Cell 1 & Cell 2 \\
\hline Machine 1 & 1 & 0 \\
Machine 2 & $\mathbf{0}$ & $\mathbf{1}$ \\
Machine 3 & 0 & 1 \\
Machine 4 & 1 & 0 \\
\hline
\end{tabular}

TABLe 15: Final matrix $M \times C$.

\begin{tabular}{lcc}
\hline & Cell 1 & Cell 2 \\
\hline Machine 1 & 1 & 0 \\
Machine 2 & 1 & 0 \\
Machine 3 & 0 & 1 \\
Machine 4 & 1 & 0 \\
\hline
\end{tabular}

Generate Initial Solutions (Stage 2 in Figure 3). At this stage, we generate initial solutions according to the method described in Section 5.1. These solutions must satisfy the three constraints of model MPCFP.

Create Initial Flock (Stage 3 in Figure 3). From the initial solutions, we create an array of birds, and finally ordered arrangement created the conceptual V-formation birds. For it, we assign the first position (first solution) in the array as the leader of the flock, and we perform an alternate distribution of birds between each side of the flock for the rest of the birds. We must consider that the birds are always sorted by fitness from low to high, the bird with lower fitness being the best solution to the problem (MPCFP is a minimization problem). Furthermore, we must consider that this is a stage in which we incorporate parallel order by quick sort and merge sort.

5.3.2. A Tour Process. This phase has two subphases internally: evolution of the leader bird and the evolution of the other birds. The evolution of the leader bird phase has one stage (generating neighboring solutions for the leader) and the evolution of the other birds phase has two stages (generating neighboring solutions for the other birds and sharing neighboring solutions that are not used by the leader with the other birds).

Generate Neighboring Solutions for the Leader (Stage 4 in Figure 3). This stage consists of generating neighboring solutions according to the steps outlined in Section 5.2. Subsequently, these neighboring solutions are sorted using an algorithm of parallel sort; see line (5) in the algorithm MBO (Algorithm 1).

Generate Neighboring Solutions for the Other Birds (Stage 5 in Figure 3). In this stage, we create $k-x$ neighboring solution to each bird of the flock; then these birds are sorted by an algorithm of parallel sort; see lines (7) and (8) in the algorithm MBO (Algorithm 1).

Share Neighboring Solutions That Are Not Used by the Leader with the Other Birds (Stage 6 in Figure 3). Share the other neighboring solutions of the leader with the other sides of the $\mathrm{V}$-formation of birds; this is done alternately from left to right or from right to left. After sharing the neighboring solutions, we performed a sorting using an algorithm of parallel sort in all neighboring solutions that every bird in the flock has (not including the leader).

Leader Replacement (Stage 7 in Figure 3). At this stage, we performed a replacement of leader; for this we choose the nearest successor; that is, choose one of the birds closer to the leader. The successor will take its place and the leader will be available at the end of the flock. We must take into consideration that if we choose a successor belonging to the left section of the flock, the leader will pass to the end of the left section. The same procedure applies to the right section of the flock (see Algorithm 2).

Algorithm Finalization (Stage 8 in Figure 3). In this step, the fitness of the leader is obtained; in addition, a procedure to remove a response matrix explicitly is made; that is, we construct an incidence matrix $A[M][P]$ according to the order given by the matrices of solutions $Y$ and $Z$. The order is according to the index of less than most of the machines and parts (see Algorithm 3). 


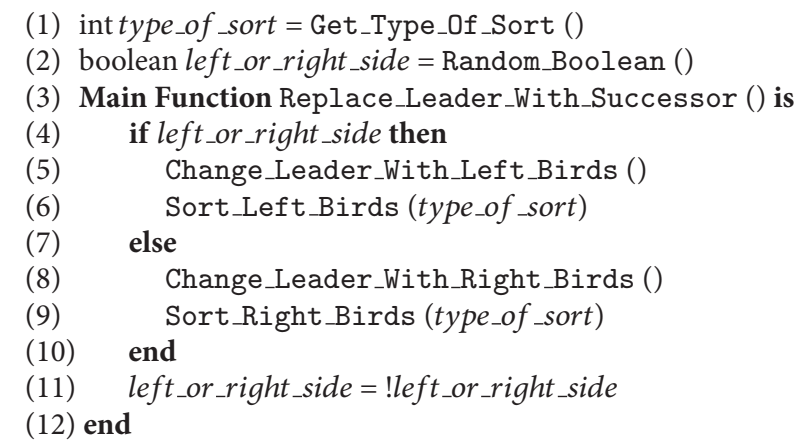

Algorithm 2: Replace leader with successor.

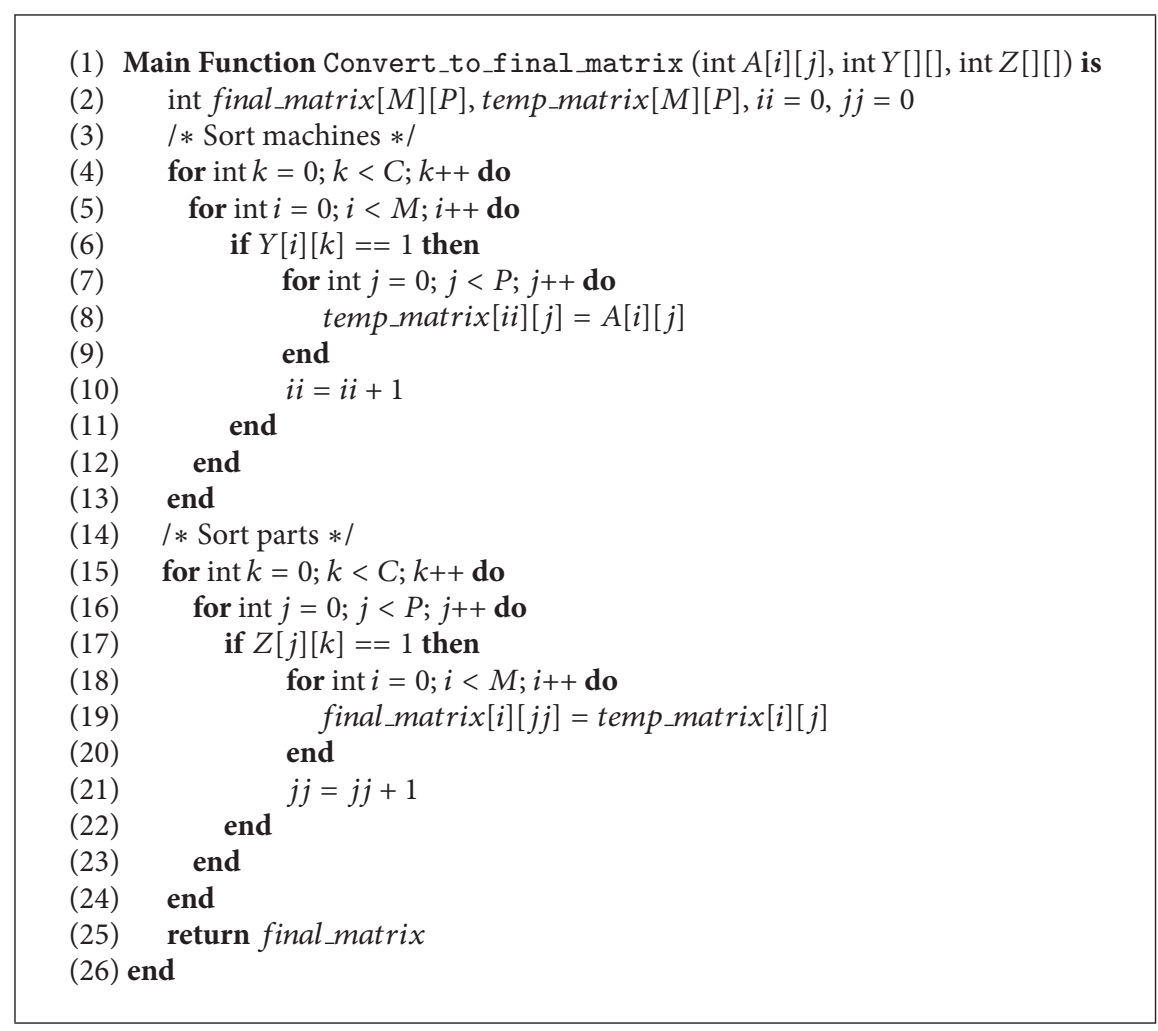

Algorithm 3: Convert matrix $M \times C$ and $P \times C$ to final matrix.

5.3.3. Sorting Algorithm. For MBO, we use two sorting algorithms, merge sort and quick sort, in which we executed sequentially and in parallel using threads.

Merge Sort. It was developed in 1945 by John Von Neumann. It is a search algorithm of complexity $O(n \log n)($ see Table 16). Algorithm 4 illustrates a general pseudocode for merge sort.

Quick Sort. It was developed in 1959 by Charles Richard Hoare. It is a search algorithm of complexity $O(n \log n)$ (see Table 16). Algorithm 5 illustrates a general pseudocode for quick sort.

\section{Computational Experiments}

6.1. Configuration Benchmarks. The MPCFP performance was evaluated experimentally using 90 test instances from Boctor's experiments, composed of 10 problems considering 5 values of $M_{\max }=8,9,10,11,12$ with $C=2$ and 10 instances considering 4 values of $M_{\max }=6,7,8,9$ with $C=3$. The test data are available in [55]. The 90 instances have been executed 31 times using 12 different configurations of sequential and parallel sorting (see Table 18). We must also consider that the sorting algorithms (sequential and parallel) were applied in the following stages (see Figure 3) of MBO:

(i) Stage $3 \rightarrow$ create initial flock.

(ii) Stage $4 \rightarrow$ generate neighboring solutions for the leader. 


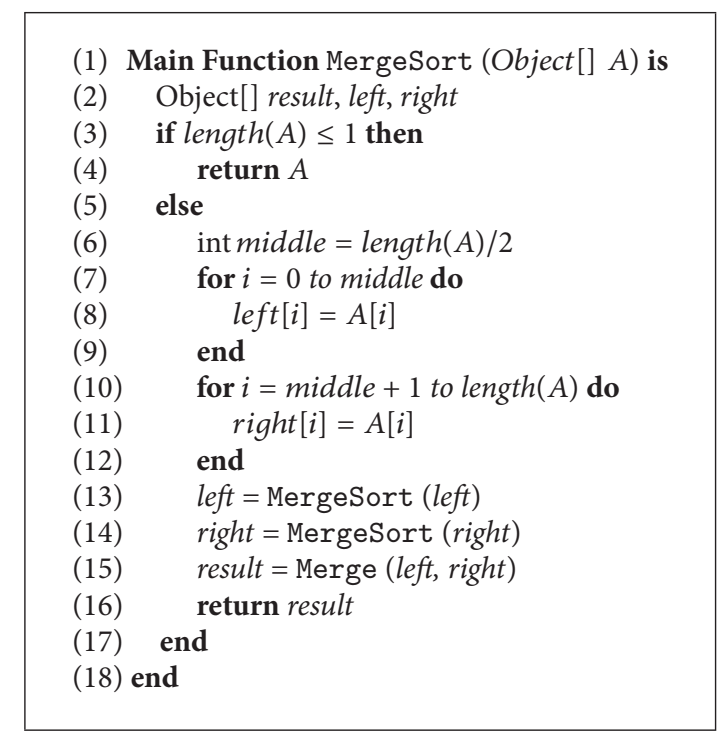

Algorithm 4: Merge sort.

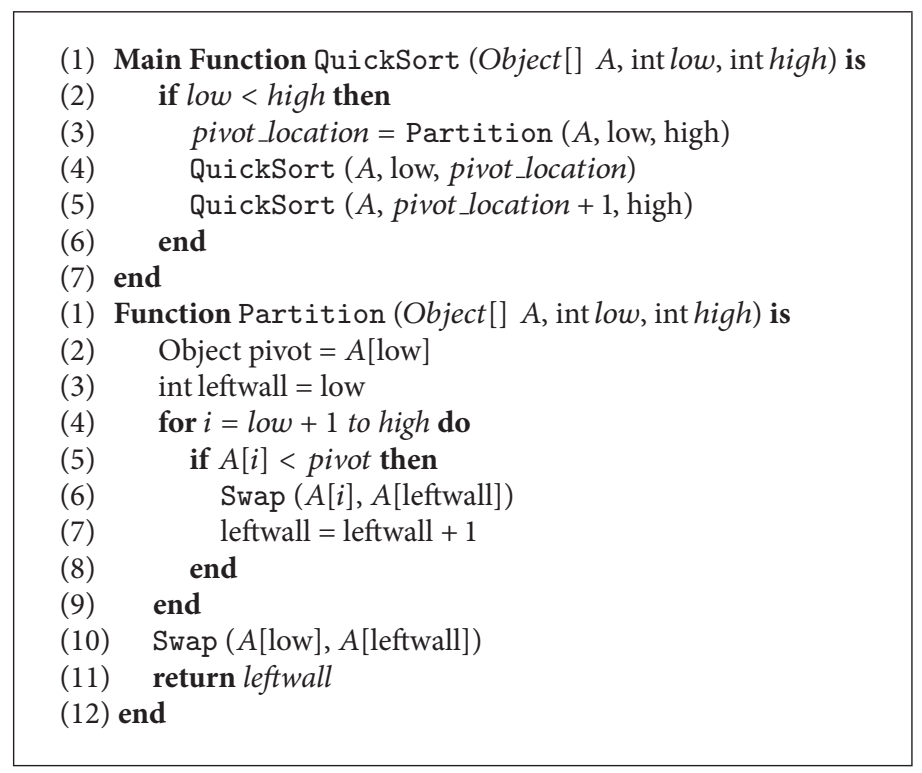

Algorithm 5: Quick sort.

(iii) Stage $5 \rightarrow$ generate neighboring solutions for other birds.

(iv) Stage $6 \rightarrow$ share neighboring solutions that are not used by the leader with the other birds.

The optimization algorithm using parallel sorting was coded in Java version 1.8.0_40 using Eclipse IDE for Java Developers Version Mars.1 Release (4.5.1). For running the MBO with parallel sorting, we use Java(TM) SE Runtime Environment (build 1.8.0_40-b27). The parameter values of $\mathrm{MBO}$ are described in Table 17. Finally, the hardware features that have run instances are MacBook Pro computer (Retina, 13-inch, late 2013) with an Intel Core i5 (processor speed = $2,4 \mathrm{GHz}$; number of processors $=1$; total number of cores = 2; $\mathrm{L} 2$ cache per core $=256 \mathrm{~KB}$; L3 cache $=3 \mathrm{MB}), 4$ GB RAM
$1600 \mathrm{MHz}$ DDR3, and Video Card Intel Iris $1536 \mathrm{MB}$ running OS X El Capitan version 10.11.2 (15C50).

6.2. Results. Tables 19 and 20 represent a summary of the results provided in Appendix A [55]. These summary tables describe the average results of 90 instances. Column 1 (threads) corresponds to the identifier assigned to the number of threads used in the search algorithm. Column 2 (execution time-BET) shows the best execution time obtained in milliseconds. Column 3 (execution time-AVG) describes the average time in milliseconds of the finest time of execution of MBO. Column 4 (execution time-ST) represents the standard deviation of the best times of execution obtained by MBO. Column 5 (cycle-BC) shows the cycle in which the optimal value was found. Column 6 (cycle-AVG) 
TABLE 16: Complexity sorting algorithm.

\begin{tabular}{|c|c|c|c|c|c|c|}
\hline Name & Best & Average & Worst & Memory & Method & Stable \\
\hline Merge sort & $O(n \log n)$ & $O(n \log n)$ & $O(n \log n)$ & Depends & Merging & Yes \\
\hline Quick sort & $O(n \log n)$ & $O(n \log n)$ & $O\left(n^{2}\right)$ & $O(\log n)$ & Partition & Depends \\
\hline
\end{tabular}

TABLE 17: Configuration parameters for MBO.

\begin{tabular}{lcc}
\hline Name & Parameter & Value \\
\hline Number of birds & $n$ & 5 \\
Number of neighbors & $k$ & 3 \\
Number of tours & $m$ & 10 \\
Number of shared solutions & $x$ & 1 \\
Number of iterations & $I$ & 2591 \\
Leader exchange mode & - & Exchange the leader with the successor \\
Sort type applied in the initial birds flock & - & Birds ordered according to their fitness \\
\hline
\end{tabular}

TABLE 18: Configuration sorting algorithms.

\begin{tabular}{|c|c|c|c|c|c|c|}
\hline \multirow{2}{*}{ Algorithm } & \multirow{2}{*}{ Sequential } & \multicolumn{5}{|c|}{ Threads } \\
\hline & & 1 & 4 & 8 & 16 & 32 \\
\hline Merge sort & Yes & Yes & Yes & Yes & Yes & Yes \\
\hline Quick sort & Yes & Yes & Yes & Yes & Yes & Yes \\
\hline
\end{tabular}

describes the average cycle. Column 7 (cycle-ST) represents the standard deviation. Column 8 (time cycle-BTC) shows the best cycle time in which the optimal value was found in milliseconds. Column 9 (time cycle-AVG) describes the average cycle in milliseconds. Column 10 (time cycle-ST) represents the standard deviation in milliseconds. Finally, we must consider all tests that have achieved the global optimum.

Execution Time Analysis. The execution time is the total time when the metaheuristic was searching in the search space for the best solution. In this sense, according to what is described in Tables 19 and 20, we can consider that the merge sort algorithm using 8 threads had the lowest average time $(152.7333 \mathrm{~ms})$ to run 90 instances (see column 2: execution time-BET in Tables 19 and 20). In addition, when we compare the times of running the metaheuristic sequentially (without threads and using merge sort algorithm), regarding the best time to run the metaheuristic reached with threads ( 8 threads), we can see that using 8 threads is an improvement in the execution time of the metaheuristic. This behavior can also be seen in Tables 19 and 20 (see column 3: execution timeAVG in Tables 19 and 20).

Cycle Analysis. A cycle is fulfilled after replacing the leader of the flock (stage 7 in Figure 3). Currently with a parameter of 2591 iterations $(I)$, we can achieve 38 cycles. Consequently, the leader of the flock during the algorithm changes its value 38 times. Basically, the cycles, where the best value has been obtained before the execution is finished, provided us with information to reduce the iteration time assigned in the input parameter of the metaheuristic. Considering the values of the cycles (see column 6: cycle-AVG in Tables 19 and 20), we can approximate an average value in 9 , for the number of cycles to find a global optimum, for using both merge sort and quick sort, with threads and without threads. Also, this involves reducing the value of the iteration limit $I=2591 \rightarrow I=$ 561. But we must also consider the random factor having the metaheuristic (see stage 2 in Figure 3, stage 4 in Figure 3, and stage 5 in Figure 3), and this randomness allows the possibility of finding a global optimum with fewer cycles (see column 5: cycle-BC in Tables 19 and 20). Therefore, this is a recommended change, but it is not enough to always find a global optimum. The quick sort algorithm using threads having irregular times is even much worse than the quick sort used sequentially.

Time Cycle Analysis. In relation to the average cycle time where the best optimum was found, we note that, according to the tests, the merge sort algorithm with 8 threads has been the least time used (see column 9: time cycle-AVG in Tables 19 and 20. It should be considered interesting results as it has achieved better times using parallel sort versus sequential sort, mainly by using merge sort with 4,8 , and 16 threads and quick sort with 16 threads (see column 8: time cycle-BTC in Tables 19 and 20).

\section{Conclusions}

In this paper, we use a Migrating Birds Optimization algorithm by a parallel sorting to solve Machine-Part Cell Formation Problems. Computational experiments using conventional hardware resources have been conducted in 90 test instances, 2 well-known sorting algorithms, 1 sequential configuration, and 5 thread configurations $(1,4,8,16$, and 32), giving a total of 1080 benchmarks. Among the main results, 
TABLE 19: Result of merge sort.

\begin{tabular}{|c|c|c|c|c|c|c|c|c|c|}
\hline \multirow{2}{*}{ Threads } & \multicolumn{3}{|c|}{ Execution time (ms) } & \multicolumn{3}{|c|}{ Cycle } & \multicolumn{3}{|c|}{ Time cycle (ms) } \\
\hline & BET & AVG & ST & $\mathrm{BC}$ & AVG & SD & BTC & AVG & SD \\
\hline Without threads & 153.9667 & 171.1556 & 21.1539 & 3.1667 & 9.0778 & 4.7263 & 13.6778 & 43.3778 & 23.8747 \\
\hline 1 & 155.5556 & 168.4111 & 13.9276 & 3.0222 & 9.0778 & 4.7812 & 13.2333 & 42.6556 & 22.9180 \\
\hline 4 & 153.9333 & 170.9556 & 28.8802 & 2.8556 & 9.1556 & 4.9501 & 12.1444 & 43.7333 & 25.6019 \\
\hline 8 & 152.7333 & 155.8667 & 3.8451 & 2.9667 & 9.2778 & 4.8785 & 12.2111 & 40.2333 & 20.2717 \\
\hline 16 & 153.1333 & 161.8222 & 11.5292 & 2.9222 & 9.1222 & 4.9112 & 12.2444 & 40.9000 & 21.5416 \\
\hline 32 & 153.9222 & 169.5000 & 21.8506 & 3.1889 & 9.2000 & 4.8937 & 13.5667 & 43.6333 & 24.7712 \\
\hline Minimum & 152.7333 & 155.8667 & 3.8451 & 2.8556 & 9.0778 & 4.7263 & 12.1444 & 40.2333 & 20.2717 \\
\hline Maximum & 155.5556 & 171.1556 & 28.8802 & 3.1889 & 9.2778 & 4.9501 & 13.6778 & 43.7333 & 25.6019 \\
\hline
\end{tabular}

TABLE 20: Result of quick sort.

\begin{tabular}{|c|c|c|c|c|c|c|c|c|c|}
\hline \multirow{2}{*}{ Threads } & \multicolumn{3}{|c|}{ Execution time (ms) } & \multicolumn{3}{|c|}{ Cycle } & \multicolumn{3}{|c|}{ Time cycle (ms) } \\
\hline & BET & AVG & ST & $\mathrm{BC}$ & AVG & $\mathrm{SD}$ & BTC & AVG & SD \\
\hline Without threads & 153.3333 & 163.0000 & 12.7284 & 2.9889 & 9.0111 & 4.9168 & 12.5222 & 41.2222 & 22.1071 \\
\hline 1 & 154.5778 & 167.9000 & 15.5926 & 3.0889 & 9.0778 & 4.8703 & 13.1778 & 42.2667 & 22.3487 \\
\hline 4 & 153.3111 & 163.6778 & 12.2109 & 3.1333 & 9.2000 & 4.9019 & 13.3222 & 41.7444 & 22.4733 \\
\hline 8 & 169.2889 & 195.6667 & 26.3893 & 2.8667 & 9.1667 & 4.8796 & 13.5667 & 49.6556 & 27.5041 \\
\hline 16 & 154.3444 & 169.7778 & 18.9543 & 2.8111 & 8.9889 & 4.7619 & 12.1333 & 42.2444 & 23.6231 \\
\hline 32 & 156.0889 & 176.7222 & 25.8717 & 2.9889 & 9.1111 & 4.8993 & 13.4111 & 45.5889 & 25.9852 \\
\hline Minimum & 153.3111 & 163.0000 & 12.2109 & 2.8111 & 8.9889 & 4.7619 & 12.1333 & 41.2222 & 22.1071 \\
\hline Maximum & 169.2889 & 195.6667 & 26.3893 & 3.1333 & 9.2000 & 4.9168 & 13.5667 & 49.6556 & 27.5041 \\
\hline
\end{tabular}

we highlight a new alignment of the MBO parameters to solve MPCFP. The new configuration consists of the following parameter values: number of birds $(n \rightarrow 5)$, number of neighbors $(k \rightarrow 3)$, number of tours $(m \rightarrow 10)$, number of shared solutions $(x \rightarrow 1)$, and number of iterations $(I \rightarrow$ 2591). We observed a decrease in cycles from 38 to 9 , which means that the leader of the flock reaching the cycle number 9 has a very high probability of finding the global optimum; therefore, we can reduce the number of iteration limit from $I=2591$ to $I=561$. This observation of the number of cycles applied to all benchmarks.

In addition, it is important to note that quick sort algoritm has variable times in the execution of MBO (see column 9: time cycle-AVG in Table 20). There have been cases in which quick sort algorithm runs without threads; it is even more favorable than when run with threads. Otherwise, with the merge sort algorithm, we found promising results. We can consider that the merge sort algorithm using 8 threads shows a reduction in execution time compared to using sequential sorting algorithm.

Among the guidelines for future research, we can point to applying different ways of generating initial solutions as well as of generating neighboring solutions. We can also compare them with other sorting algorithms implemented in MBO.

\section{Appendix}

\section{A. Details of the Benchmarks}

A.1. Boctor's Problems. Table 21 describes the configuration of the 90 instances of tests. The description of the table is given the following attributes: Column 1 (ID) corresponds to the identifier assigned to each instance. Column 2 (Boctor's problem) represents the identifier of the 10 Boctor's problems. Column 3 (cell) is the number of cells. Column $4\left(M_{\max }\right)$ corresponds to the maximum number of machines per cell. Column 5 (OPT) depicts the optimum global for the given problem.

A.2. Experimental Results. Tables 22, 23, 24, 25, 26, 27, 28, $29,30,31,32$, and 33 constrast the times by using different sorting algorithms. These tables have the same headers, which are described below: column 1 (ID) corresponds to the identifier assigned to each instance. Column 2 (OPT) depicts the optimum value for the given problem. Column 3 (optimum-MBO) depicts the best value reached by using Migrating Birds Optimization. Column 4 (optimum-AVG) the average value of 31 executiones is depicted. Column 5 (optimum-SD) represents the standard deviation. Column 6 (optimum-RPD\%) represents the difference between the best known optimum value and the best optimum value reached by $\mathrm{MBO}$ in terms of percentage. Column 7 (execution time-BET) shows the best execution time obtained in milliseconds. Column 8 (execution time-AVG) describes the average time in milliseconds of the finest time of execution of MBO. Column 9 (execution time-ST) represents the standard deviation of the best times of execution obtained by MBO. Column 10 (cycle-BC) shows the cycle in which the optimal value was found. Column 11 (cycle-AVG) describes the average cycle. Column 12 (cycle-ST) represents the standard deviation. Column 13 (time cycle-BTC) shows the best cycle 
TABLE 21: Boctor's problems.

\begin{tabular}{|c|c|c|c|c|}
\hline ID & Boctor's problem & Cell & $M_{\max }$ & OPT \\
\hline $\mathrm{B} 01$ & 1 & 2 & 8 & 11 \\
\hline B02 & 1 & 2 & 9 & 11 \\
\hline B03 & 1 & 2 & 10 & 11 \\
\hline B04 & 1 & 2 & 11 & 11 \\
\hline B05 & 1 & 2 & 12 & 11 \\
\hline B06 & 2 & 2 & 8 & 7 \\
\hline B07 & 2 & 2 & 9 & 6 \\
\hline B08 & 2 & 2 & 10 & 4 \\
\hline B09 & 2 & 2 & 11 & 3 \\
\hline $\mathrm{B} 10$ & 2 & 2 & 12 & 3 \\
\hline B11 & 3 & 2 & 8 & 4 \\
\hline B12 & 3 & 2 & 9 & 4 \\
\hline $\mathrm{B} 13$ & 3 & 2 & 10 & 4 \\
\hline B14 & 3 & 2 & 11 & 3 \\
\hline B15 & 3 & 2 & 12 & 1 \\
\hline B16 & 4 & 2 & 8 & 14 \\
\hline B17 & 4 & 2 & 9 & 13 \\
\hline $\mathrm{B} 18$ & 4 & 2 & 10 & 13 \\
\hline B19 & 4 & 2 & 11 & 13 \\
\hline B20 & 4 & 2 & 12 & 13 \\
\hline B21 & 5 & 2 & 8 & 9 \\
\hline B22 & 5 & 2 & 9 & 6 \\
\hline B23 & 5 & 2 & 10 & 6 \\
\hline B24 & 5 & 2 & 11 & 5 \\
\hline B25 & 5 & 2 & 12 & 4 \\
\hline B26 & 6 & 2 & 8 & 5 \\
\hline B27 & 6 & 2 & 9 & 3 \\
\hline B28 & 6 & 2 & 10 & 3 \\
\hline B29 & 6 & 2 & 11 & 3 \\
\hline B30 & 6 & 2 & 12 & 2 \\
\hline B31 & 7 & 2 & 8 & 7 \\
\hline B32 & 7 & 2 & 9 & 4 \\
\hline B33 & 7 & 2 & 10 & 4 \\
\hline B34 & 7 & 2 & 11 & 4 \\
\hline B35 & 7 & 2 & 12 & 4 \\
\hline B36 & 8 & 2 & 8 & 13 \\
\hline B37 & 8 & 2 & 9 & 10 \\
\hline B38 & 8 & 2 & 10 & 8 \\
\hline B39 & 8 & 2 & 11 & 5 \\
\hline B40 & 8 & 2 & 12 & 5 \\
\hline B41 & 9 & 2 & 8 & 8 \\
\hline B42 & 9 & 2 & 9 & 8 \\
\hline B43 & 9 & 2 & 10 & 8 \\
\hline B44 & 9 & 2 & 11 & 5 \\
\hline B45 & 9 & 2 & 12 & 5 \\
\hline B46 & 10 & 2 & 8 & 8 \\
\hline B47 & 10 & 2 & 9 & 5 \\
\hline B48 & 10 & 2 & 10 & 5 \\
\hline B49 & 10 & 2 & 11 & 5 \\
\hline B50 & 10 & 2 & 12 & 5 \\
\hline B51 & 1 & 3 & 6 & 27 \\
\hline
\end{tabular}

TABLE 21: Continued.

\begin{tabular}{lcccc}
\hline ID & Boctor's problem & Cell & $M_{\max }$ & OPT \\
\hline B52 & 1 & 3 & 7 & 18 \\
B53 & 1 & 3 & 8 & 11 \\
B54 & 1 & 3 & 9 & 11 \\
B55 & 2 & 3 & 6 & 7 \\
B56 & 2 & 3 & 7 & 6 \\
B57 & 2 & 3 & 8 & 6 \\
B58 & 2 & 3 & 9 & 6 \\
B59 & 3 & 3 & 6 & 9 \\
B60 & 3 & 3 & 7 & 4 \\
B61 & 3 & 3 & 8 & 4 \\
B62 & 3 & 3 & 9 & 4 \\
B63 & 4 & 3 & 6 & 27 \\
B64 & 4 & 3 & 7 & 18 \\
B65 & 4 & 3 & 8 & 14 \\
B66 & 4 & 3 & 9 & 13 \\
B67 & 5 & 3 & 6 & 11 \\
B68 & 5 & 3 & 7 & 8 \\
B69 & 5 & 3 & 8 & 8 \\
B70 & 5 & 3 & 9 & 6 \\
B71 & 6 & 3 & 6 & 6 \\
B72 & 6 & 3 & 7 & 4 \\
B73 & 6 & 3 & 8 & 4 \\
B74 & 6 & 3 & 9 & 3 \\
B75 & 7 & 3 & 6 & 11 \\
B76 & 7 & 3 & 7 & 5 \\
B77 & 7 & 3 & 8 & 5 \\
B78 & 7 & 3 & 9 & 4 \\
B79 & 8 & 3 & 6 & 14 \\
B80 & 8 & 3 & 7 & 11 \\
B81 & 8 & 3 & 8 & 11 \\
B82 & 8 & 3 & 9 & 10 \\
B83 & 9 & 3 & 6 & 12 \\
B84 & 9 & 3 & 7 & 12 \\
B85 & 9 & 3 & 8 & 8 \\
B86 & 9 & 3 & 9 & 8 \\
B87 & 10 & 3 & 6 & 10 \\
B88 & 10 & 3 & 7 & 8 \\
B89 & 10 & 3 & 8 & 8 \\
B90 & 10 & 3 & 9 & 5 \\
\hline & & & & \\
\hline
\end{tabular}

time in which the optimal value was found in milliseconds. Column 14 (time cycle-AVG) describes the average cycle in milliseconds. Column 15 (time cycle-ST) represents the standard deviation in milliseconds. Finally, it describes the average time for each column.

\section{Competing Interests}

The authors declare that they have no competing interests regarding the publication of this paper. 
TABLE 22: MBO using sequential merge sort algorithm.

\begin{tabular}{|c|c|c|c|c|c|c|c|c|c|c|c|c|c|c|}
\hline \multirow{2}{*}{ ID } & \multirow{2}{*}{ OPT } & \multicolumn{4}{|c|}{ Optimum } & \multicolumn{3}{|c|}{ Execution time } & \multicolumn{3}{|c|}{ Cycle } & \multicolumn{3}{|c|}{ Time cycle } \\
\hline & & $\mathrm{MBO}$ & AVG & SD & RPD\% & BET & AVG & ST & $\mathrm{BC}$ & AVG & SD & BTC & AVG & SD \\
\hline $\mathrm{B} 1$ & 11 & 11 & 11.0000 & 0.0000 & 0.00 & 158 & 251 & 171.6413 & 4 & 7 & 2.4350 & 17 & 53 & 34.2198 \\
\hline B2 & 11 & 11 & 11.0000 & 0.0000 & 0.00 & 157 & 263 & 84.9052 & 1 & 3 & 1.1575 & 4 & 31 & 23.2120 \\
\hline B3 & 11 & 11 & 11.0000 & 0.0000 & 0.00 & 153 & 209 & 47.3905 & 2 & 4 & 1.4984 & 9 & 24 & 10.2378 \\
\hline B4 & 11 & 11 & 11.0000 & 0.0000 & 0.00 & 155 & 160 & 4.7848 & 2 & 4 & 1.9449 & 8 & 20 & 8.5007 \\
\hline B5 & 11 & 11 & 11.0000 & 0.0000 & 0.00 & 155 & 160 & 4.4053 & 2 & 4 & 2.0723 & 8 & 20 & 9.2034 \\
\hline B6 & 7 & 7 & 7.0000 & 0.0000 & 0.00 & 165 & 172 & 5.9968 & 2 & 8 & 5.4244 & 10 & 40 & 25.3084 \\
\hline B7 & 6 & 6 & 6.0000 & 0.0000 & 0.00 & 158 & 165 & 10.2942 & 2 & 5 & 2.6567 & 9 & 24 & 10.9301 \\
\hline B8 & 4 & 4 & 4.1935 & 0.6011 & 0.00 & 154 & 226 & 63.7678 & 3 & 10 & 8.5001 & 13 & 59 & 55.3723 \\
\hline B9 & 3 & 3 & 3.1935 & 0.4016 & 0.00 & 152 & 157 & 3.4802 & 2 & 5 & 2.5686 & 8 & 24 & 10.5719 \\
\hline $\mathrm{B} 10$ & 3 & 3 & 3.0000 & 0.0000 & 0.00 & 152 & 183 & 41.7133 & 2 & 4 & 1.5422 & 8 & 21 & 10.5715 \\
\hline B11 & 4 & 4 & 4.1613 & 0.3739 & 0.00 & 155 & 233 & 66.3620 & 2 & 11 & 9.9109 & 9 & 73 & 63.6411 \\
\hline B12 & 4 & 4 & 4.0000 & 0.0000 & 0.00 & 153 & 235 & 86.2169 & 2 & 4 & 1.6643 & 11 & 29 & 18.5750 \\
\hline $\mathrm{B} 13$ & 4 & 4 & 4.0000 & 0.0000 & 0.00 & 152 & 161 & 30.0537 & 3 & 6 & 3.6577 & 12 & 27 & 14.8101 \\
\hline B14 & 3 & 3 & 3.3548 & 0.4864 & 0.00 & 152 & 159 & 26.0370 & 2 & 6 & 3.7006 & 8 & 26 & 15.6561 \\
\hline B15 & 1 & 1 & 1.6452 & 0.9504 & 0.00 & 152 & 155 & 2.6176 & 2 & 5 & 2.3505 & 8 & 22 & 9.6363 \\
\hline B16 & 14 & 14 & 14.0000 & 0.0000 & 0.00 & 155 & 182 & 48.3518 & 3 & 8 & 3.5493 & 12 & 43 & 25.2893 \\
\hline B17 & 13 & 13 & 13.0000 & 0.0000 & 0.00 & 152 & 174 & 35.1554 & 2 & 5 & 2.1717 & 8 & 26 & 9.4585 \\
\hline B18 & 13 & 13 & 13.0000 & 0.0000 & 0.00 & 152 & 177 & 41.1985 & 2 & 4 & 1.8275 & 8 & 21 & 8.6059 \\
\hline B19 & 13 & 13 & 13.0968 & 0.5388 & 0.00 & 153 & 218 & 62.5518 & 1 & 6 & 4.0377 & 4 & 39 & 26.7267 \\
\hline B20 & 13 & 13 & 13.0000 & 0.0000 & 0.00 & 155 & 225 & 138.3519 & 3 & 7 & 4.5423 & 13 & 41 & 21.4062 \\
\hline B21 & 9 & 9 & 9.1935 & 0.4016 & 0.00 & 153 & 172 & 43.6026 & 2 & 9 & 5.2990 & 8 & 41 & 26.7630 \\
\hline B22 & 6 & 6 & 6.1290 & 0.3408 & 0.00 & 152 & 155 & 2.0494 & 1 & 5 & 3.1326 & 4 & 23 & 12.9070 \\
\hline B23 & 6 & 6 & 6.1290 & 0.3408 & 0.00 & 152 & 153 & 2.0489 & 2 & 7 & 4.8491 & 8 & 31 & 19.7735 \\
\hline B24 & 5 & 5 & 5.2581 & 0.5755 & 0.00 & 152 & 153 & 1.6409 & 2 & 6 & 2.4012 & 8 & 24 & 9.6949 \\
\hline B25 & 4 & 4 & 4.1290 & 0.3408 & 0.00 & 152 & 154 & 6.0300 & 3 & 6 & 5.4158 & 12 & 27 & 22.1520 \\
\hline B26 & 5 & 5 & 5.0000 & 0.0000 & 0.00 & 154 & 157 & 2.3372 & 2 & 8 & 6.0393 & 8 & 37 & 24.7760 \\
\hline $\mathrm{B} 27$ & 3 & 3 & 3.0323 & 0.1796 & 0.00 & 152 & 154 & 2.2017 & 2 & 7 & 4.8524 & 8 & 30 & 19.3886 \\
\hline B28 & 3 & 3 & 3.2903 & 0.5884 & 0.00 & 152 & 155 & 4.7996 & 1 & 5 & 3.2670 & 4 & 24 & 13.9205 \\
\hline B29 & 3 & 3 & 3.0968 & 0.3005 & 0.00 & 152 & 156 & 7.3660 & 3 & 5 & 2.0447 & 12 & 23 & 8.2142 \\
\hline $\mathrm{B} 30$ & 2 & 2 & 2.4516 & 0.5680 & 0.00 & 152 & 153 & 1.7289 & 2 & 7 & 6.5031 & 8 & 28 & 26.3895 \\
\hline B31 & 7 & 7 & 7.0000 & 0.0000 & 0.00 & 153 & 157 & 2.7615 & 4 & 8 & 3.2156 & 19 & 37 & 13.2221 \\
\hline B32 & 4 & 4 & 4.2903 & 0.9016 & 0.00 & 152 & 155 & 4.1960 & 3 & 7 & 3.7805 & 13 & 31 & 15.0563 \\
\hline B33 & 4 & 4 & 4.1290 & 0.4995 & 0.00 & 152 & 154 & 3.7868 & 2 & 6 & 3.0154 & 8 & 26 & 12.7931 \\
\hline B34 & 4 & 4 & 4.3226 & 0.7478 & 0.00 & 152 & 153 & 1.2835 & 2 & 6 & 4.2925 & 8 & 27 & 17.1088 \\
\hline B35 & 4 & 4 & 4.7097 & 0.9727 & 0.00 & 152 & 153 & 1.6468 & 1 & 5 & 2.0723 & 4 & 21 & 8.5183 \\
\hline B36 & 13 & 13 & 13.0000 & 0.0000 & 0.00 & 154 & 158 & 3.8763 & 3 & 9 & 4.9110 & 13 & 39 & 19.3956 \\
\hline B37 & 10 & 10 & 10.3226 & 0.7478 & 0.00 & 152 & 155 & 2.5387 & 2 & 6 & 5.4497 & 9 & 28 & 22.2304 \\
\hline B38 & 8 & 8 & 8.0968 & 0.3005 & 0.00 & 152 & 154 & 3.3601 & 2 & 8 & 5.6323 & 8 & 34 & 22.8731 \\
\hline B39 & 5 & 5 & 5.1935 & 1.0776 & 0.00 & 152 & 166 & 20.9165 & 2 & 6 & 3.5493 & 8 & 27 & 15.7638 \\
\hline B40 & 5 & 5 & 5.0000 & 0.0000 & 0.00 & 152 & 162 & 16.8069 & 2 & 4 & 1.8357 & 8 & 19 & 7.9044 \\
\hline B41 & 8 & 8 & 8.0000 & 0.0000 & 0.00 & 155 & 158 & 2.9064 & 1 & 10 & 5.2974 & 4 & 42 & 22.2617 \\
\hline B42 & 8 & 8 & 8.0000 & 0.0000 & 0.00 & 152 & 198 & 70.2758 & 1 & 4 & 2.0718 & 8 & 24 & 17.2073 \\
\hline B43 & 8 & 8 & 8.0000 & 0.0000 & 0.00 & 152 & 154 & 1.7221 & 1 & 4 & 2.2818 & 4 & 19 & 9.3681 \\
\hline B44 & 5 & 5 & 5.9677 & 1.4256 & 0.00 & 152 & 154 & 3.8616 & 2 & 5 & 2.5264 & 8 & 24 & 10.1847 \\
\hline B45 & 5 & 5 & 6.1935 & 1.5366 & 0.00 & 152 & 153 & 1.3536 & 2 & 6 & 4.6467 & 8 & 26 & 19.1860 \\
\hline B46 & 8 & 8 & 8.0645 & 0.2497 & 0.00 & 154 & 158 & 3.4348 & 3 & 10 & 6.7563 & 12 & 42 & 27.9592 \\
\hline B47 & 5 & 5 & 5.0000 & 0.0000 & 0.00 & 152 & 155 & 4.1228 & 2 & 5 & 2.9256 & 8 & 22 & 13.0905 \\
\hline B48 & 5 & 5 & 5.4839 & 2.0145 & 0.00 & 152 & 153 & 1.3897 & 2 & 5 & 2.8931 & 8 & 22 & 11.7203 \\
\hline B49 & 5 & 5 & 5.1935 & 0.6011 & 0.00 & 152 & 152 & 1.1704 & 3 & 6 & 3.1819 & 12 & 26 & 12.7969 \\
\hline $\mathrm{B} 50$ & 5 & 5 & 5.3226 & 0.7478 & 0.00 & 152 & 153 & 1.4473 & 2 & 6 & 3.4529 & 8 & 26 & 14.0041 \\
\hline
\end{tabular}


TABle 22: Continued.

\begin{tabular}{|c|c|c|c|c|c|c|c|c|c|c|c|c|c|c|}
\hline \multirow{2}{*}{ ID } & \multirow{2}{*}{$\mathrm{OPT}$} & \multicolumn{4}{|c|}{ Optimum } & \multicolumn{3}{|c|}{ Execution time } & \multicolumn{3}{|c|}{ Cycle } & \multicolumn{3}{|c|}{ Time cycle } \\
\hline & & $\mathrm{MBO}$ & AVG & SD & RPD\% & BET & AVG & ST & $\mathrm{BC}$ & AVG & $\mathrm{SD}$ & BTC & AVG & SD \\
\hline B51 & 27 & 27 & 28.1613 & 0.9694 & 0.00 & 154 & 159 & 4.7774 & 5 & 21 & 9.0993 & 21 & 92 & 39.5406 \\
\hline B52 & 18 & 18 & 18.9355 & 1.0307 & 0.00 & 154 & 156 & 2.2785 & 2 & 19 & 9.3341 & 8 & 80 & 38.7324 \\
\hline B53 & 11 & 11 & 11.0000 & 0.0000 & 0.00 & 153 & 155 & 1.5889 & 4 & 10 & 2.8815 & 16 & 42 & 12.1075 \\
\hline B54 & 11 & 11 & 11.0000 & 0.0000 & 0.00 & 152 & 154 & 1.6277 & 3 & 7 & 2.4596 & 12 & 32 & 10.2627 \\
\hline B55 & 7 & 7 & 7.0000 & 0.0000 & 0.00 & 154 & 158 & 4.4897 & 4 & 11 & 5.5967 & 16 & 48 & 22.6364 \\
\hline B56 & 6 & 6 & 6.0000 & 0.0000 & 0.00 & 153 & 155 & 1.9233 & 4 & 10 & 3.4112 & 16 & 44 & 13.9193 \\
\hline B57 & 6 & 6 & 6.0968 & 0.3962 & 0.00 & 153 & 154 & 1.3552 & 6 & 12 & 5.2644 & 24 & 52 & 21.4015 \\
\hline B58 & 6 & 6 & 6.0645 & 0.3592 & 0.00 & 152 & 155 & 4.0377 & 3 & 9 & 3.7643 & 14 & 38 & 14.9752 \\
\hline B59 & 9 & 9 & 9.1290 & 0.3408 & 0.00 & 154 & 158 & 2.6825 & 5 & 15 & 6.7165 & 21 & 64 & 28.5839 \\
\hline B60 & 4 & 4 & 4.3871 & 1.2021 & 0.00 & 152 & 156 & 2.3145 & 6 & 13 & 5.7136 & 24 & 56 & 23.8186 \\
\hline B61 & 4 & 4 & 4.1290 & 0.3408 & 0.00 & 152 & 155 & 2.0394 & 4 & 13 & 6.7101 & 16 & 54 & 27.4017 \\
\hline B62 & 4 & 4 & 4.0000 & 0.0000 & 0.00 & 152 & 155 & 4.1824 & 4 & 9 & 3.4273 & 18 & 40 & 13.6191 \\
\hline B63 & 27 & 27 & 27.2581 & 0.5143 & 0.00 & 155 & 165 & 11.2005 & 4 & 21 & 11.4243 & 16 & 90 & 48.4598 \\
\hline B64 & 18 & 18 & 18.3871 & 0.9549 & 0.00 & 155 & 167 & 14.2569 & 7 & 19 & 9.3371 & 28 & 86 & 39.7379 \\
\hline B65 & 14 & 14 & 14.0645 & 0.2497 & 0.00 & 153 & 161 & 9.3472 & 6 & 15 & 7.5537 & 25 & 65 & 31.4611 \\
\hline B66 & 13 & 13 & 13.0000 & 0.0000 & 0.00 & 154 & 247 & 97.6943 & 6 & 11 & 3.3020 & 24 & 81 & 67.8854 \\
\hline B67 & 11 & 11 & 11.0323 & 0.1796 & 0.00 & 156 & 209 & 33.3034 & 4 & 14 & 7.7241 & 19 & 77 & 43.7733 \\
\hline B68 & 8 & 8 & 8.8065 & 2.3154 & 0.00 & 156 & 179 & 30.7013 & 2 & 13 & 7.7239 & 9 & 63 & 40.9466 \\
\hline B69 & 8 & 8 & 8.5161 & 0.6256 & 0.00 & 154 & 190 & 35.2266 & 5 & 12 & 5.7914 & 20 & 64 & 35.7448 \\
\hline B70 & 6 & 6 & 6.4839 & 0.6256 & 0.00 & 152 & 167 & 16.6121 & 5 & 12 & 5.6220 & 22 & 57 & 26.9089 \\
\hline B71 & 6 & 6 & 6.0000 & 0.0000 & 0.00 & 155 & 176 & 15.3473 & 6 & 11 & 5.4370 & 29 & 54 & 24.4164 \\
\hline B72 & 4 & 4 & 4.2258 & 0.5603 & 0.00 & 155 & 168 & 21.0614 & 6 & 12 & 4.0783 & 28 & 55 & 23.1119 \\
\hline B73 & 4 & 4 & 4.4516 & 0.6752 & 0.00 & 152 & 173 & 15.2415 & 4 & 11 & 6.4611 & 16 & 52 & 28.1919 \\
\hline B74 & 3 & 3 & 3.3226 & 0.4752 & 0.00 & 153 & 166 & 14.9468 & 4 & 13 & 8.6221 & 20 & 60 & 35.3722 \\
\hline B75 & 11 & 11 & 11.6774 & 0.9794 & 0.00 & 156 & 169 & 8.4222 & 4 & 17 & 10.5949 & 17 & 77 & 47.6167 \\
\hline B76 & 5 & 5 & 5.2581 & 0.6816 & 0.00 & 154 & 167 & 23.7204 & 3 & 12 & 5.0513 & 14 & 56 & 26.0949 \\
\hline B77 & 5 & 5 & 5.3871 & 0.8032 & 0.00 & 165 & 174 & 8.3753 & 6 & 14 & 8.1313 & 27 & 66 & 37.9381 \\
\hline B78 & 4 & 4 & 4.9355 & 1.2093 & 0.00 & 163 & 176 & 12.2235 & 5 & 13 & 4.7399 & 22 & 60 & 25.3983 \\
\hline B79 & 14 & 14 & 14.0323 & 0.1796 & 0.00 & 156 & 181 & 28.4157 & 5 & 10 & 3.2965 & 25 & 47 & 16.9919 \\
\hline B80 & 11 & 11 & 11.0000 & 0.0000 & 0.00 & 171 & 190 & 20.4805 & 3 & 10 & 4.6704 & 17 & 53 & 23.3860 \\
\hline B81 & 11 & 11 & 11.3871 & 0.8032 & 0.00 & 159 & 186 & 24.6086 & 7 & 14 & 7.1159 & 34 & 70 & 35.5031 \\
\hline B82 & 10 & 10 & 10.3548 & 0.6607 & 0.00 & 153 & 169 & 25.0386 & 4 & 12 & 6.0875 & 16 & 55 & 29.4125 \\
\hline B83 & 12 & 12 & 12.2258 & 1.2572 & 0.00 & 154 & 167 & 8.4371 & 4 & 12 & 6.3860 & 18 & 57 & 29.6714 \\
\hline B84 & 12 & 12 & 12.0000 & 0.0000 & 0.00 & 155 & 165 & 13.7350 & 6 & 11 & 3.8852 & 31 & 52 & 17.8962 \\
\hline B85 & 8 & 8 & 8.9355 & 1.4361 & 0.00 & 153 & 170 & 23.4146 & 4 & 14 & 7.3518 & 17 & 64 & 33.0030 \\
\hline B86 & 8 & 8 & 8.1613 & 0.5829 & 0.00 & 156 & 177 & 15.4220 & 3 & 10 & 3.7814 & 13 & 47 & 18.0706 \\
\hline B87 & 10 & 10 & 10.4839 & 1.0605 & 0.00 & 156 & 184 & 20.5574 & 4 & 18 & 9.4481 & 19 & 89 & 50.8878 \\
\hline B88 & 8 & 8 & 8.0000 & 0.0000 & 0.00 & 155 & 194 & 109.8234 & 4 & 9 & 3.1995 & 20 & 61 & 96.8101 \\
\hline B89 & 8 & 8 & 8.0000 & 0.0000 & 0.00 & 153 & 159 & 8.7550 & 4 & 10 & 3.7275 & 17 & 42 & 15.4842 \\
\hline \multirow[t]{2}{*}{ B90 } & 5 & 5 & 5.2903 & 0.9016 & 0.00 & 152 & 156 & 2.2509 & 4 & 11 & 5.6435 & 17 & 49 & 22.9895 \\
\hline & & AVG & 8.1025 & 0.4576 & 0.00 & 153 & 171 & 21.1539 & 3 & 9 & 4.7263 & 13 & 43 & 23.8747 \\
\hline
\end{tabular}


TABLE 23: MBO using parallel merge sort algorithm with 1 thread.

\begin{tabular}{|c|c|c|c|c|c|c|c|c|c|c|c|c|c|c|}
\hline \multirow{2}{*}{ ID } & \multirow{2}{*}{ OPT } & \multicolumn{4}{|c|}{ Optimum } & \multicolumn{3}{|c|}{ Execution time } & \multicolumn{3}{|c|}{ Cycle } & \multicolumn{3}{|c|}{ Time cycle } \\
\hline & & $\mathrm{MBO}$ & AVG & $\mathrm{SD}$ & RPD\% & BET & AVG & ST & $\mathrm{BC}$ & AVG & $\mathrm{SD}$ & BTC & AVG & $\mathrm{SD}$ \\
\hline $\mathrm{B} 1$ & 11 & 11 & 11.0000 & 0.0000 & 0.00 & 159 & 206 & 78.6150 & 2 & 6 & 3.2038 & 14 & 43 & 35.4355 \\
\hline B2 & 11 & 11 & 11.0000 & 0.0000 & 0.00 & 155 & 173 & 19.0187 & 1 & 4 & 1.5141 & 6 & 21 & 8.1043 \\
\hline B3 & 11 & 11 & 11.0000 & 0.0000 & 0.00 & 154 & 172 & 13.6524 & 1 & 3 & 1.2495 & 4 & 16 & 5.5298 \\
\hline B4 & 11 & 11 & 11.0000 & 0.0000 & 0.00 & 153 & 172 & 16.4752 & 3 & 5 & 1.6552 & 12 & 24 & 10.3208 \\
\hline B5 & 11 & 11 & 11.0000 & 0.0000 & 0.00 & 152 & 155 & 5.0775 & 2 & 5 & 3.1064 & 8 & 24 & 12.5736 \\
\hline B6 & 7 & 7 & 7.0000 & 0.0000 & 0.00 & 155 & 185 & 36.0141 & 3 & 10 & 6.0544 & 13 & 48 & 27.2948 \\
\hline B7 & 6 & 6 & 6.0000 & 0.0000 & 0.00 & 159 & 202 & 24.7416 & 2 & 6 & 4.4252 & 9 & 35 & 21.5014 \\
\hline B8 & 4 & 4 & 4.4516 & 0.8500 & 0.00 & 158 & 197 & 88.5635 & 3 & 12 & 8.1669 & 17 & 58 & 40.5206 \\
\hline B9 & 3 & 3 & 3.0645 & 0.2497 & 0.00 & 153 & 172 & 12.9714 & 2 & 5 & 2.3122 & 12 & 23 & 9.5575 \\
\hline $\mathrm{B} 10$ & 3 & 3 & 3.0000 & 0.0000 & 0.00 & 152 & 168 & 13.5060 & 1 & 4 & 2.2609 & 7 & 20 & 10.8349 \\
\hline B11 & 4 & 4 & 4.2258 & 0.4250 & 0.00 & 156 & 186 & 18.4762 & 1 & 10 & 7.4314 & 5 & 53 & 41.0204 \\
\hline B12 & 4 & 4 & 4.0000 & 0.0000 & 0.00 & 160 & 178 & 17.7416 & 2 & 4 & 1.7585 & 8 & 21 & 10.0164 \\
\hline $\mathrm{B} 13$ & 4 & 4 & 4.0000 & 0.0000 & 0.00 & 152 & 171 & 19.3874 & 2 & 4 & 2.2714 & 8 & 21 & 10.1196 \\
\hline B14 & 3 & 3 & 3.2903 & 0.4614 & 0.00 & 152 & 169 & 15.2222 & 2 & 6 & 4.9287 & 8 & 28 & 22.3718 \\
\hline B15 & 1 & 1 & 1.0968 & 0.5388 & 0.00 & 153 & 165 & 11.9830 & 2 & 6 & 3.4694 & 8 & 29 & 16.5698 \\
\hline B16 & 14 & 14 & 14.0000 & 0.0000 & 0.00 & 155 & 163 & 9.5187 & 2 & 7 & 3.4128 & 8 & 33 & 14.8828 \\
\hline $\mathrm{B} 17$ & 13 & 13 & 13.0000 & 0.0000 & 0.00 & 153 & 155 & 2.8564 & 2 & 5 & 2.0225 & 8 & 21 & 8.8107 \\
\hline B18 & 13 & 13 & 13.0000 & 0.0000 & 0.00 & 152 & 155 & 2.6401 & 2 & 5 & 2.2264 & 8 & 24 & 9.3277 \\
\hline B19 & 13 & 13 & 13.3871 & 1.0223 & 0.00 & 152 & 155 & 3.7145 & 2 & 7 & 4.0236 & 8 & 30 & 16.7423 \\
\hline B20 & 13 & 13 & 13.0000 & 0.0000 & 0.00 & 152 & 154 & 2.2240 & 2 & 6 & 3.6308 & 8 & 28 & 14.7566 \\
\hline B21 & 9 & 9 & 9.1935 & 0.4016 & 0.00 & 154 & 161 & 4.3366 & 3 & 11 & 6.9041 & 12 & 47 & 29.4637 \\
\hline B22 & 6 & 6 & 6.1935 & 0.4016 & 0.00 & 153 & 156 & 2.2926 & 1 & 5 & 2.4203 & 4 & 23 & 10.0929 \\
\hline B23 & 6 & 6 & 6.0645 & 0.2497 & 0.00 & 152 & 155 & 4.3901 & 2 & 7 & 4.9174 & 8 & 31 & 19.7046 \\
\hline B24 & 5 & 5 & 5.3548 & 0.6607 & 0.00 & 152 & 155 & 2.7712 & 2 & 6 & 5.4696 & 8 & 28 & 22.2898 \\
\hline B25 & 4 & 4 & 4.3871 & 0.6672 & 0.00 & 152 & 156 & 3.3534 & 3 & 7 & 3.7411 & 12 & 29 & 15.0894 \\
\hline B26 & 5 & 5 & 5.0323 & 0.1796 & 0.00 & 155 & 162 & 5.0227 & 2 & 7 & 3.3376 & 9 & 34 & 13.9975 \\
\hline $\mathrm{B} 27$ & 3 & 3 & 3.0323 & 0.1796 & 0.00 & 154 & 157 & 4.5927 & 3 & 6 & 3.4619 & 12 & 27 & 14.2464 \\
\hline B28 & 3 & 3 & 3.0968 & 0.3005 & 0.00 & 152 & 156 & 3.8156 & 2 & 6 & 2.3714 & 8 & 25 & 10.0092 \\
\hline B29 & 3 & 3 & 3.3548 & 0.4864 & 0.00 & 152 & 155 & 3.1271 & 2 & 4 & 2.4913 & 8 & 19 & 10.2875 \\
\hline $\mathrm{B} 30$ & 2 & 2 & 2.5484 & 0.6752 & 0.00 & 154 & 157 & 3.3421 & 1 & 8 & 7.1106 & 6 & 33 & 29.8981 \\
\hline B31 & 7 & 7 & 7.0000 & 0.0000 & 0.00 & 158 & 166 & 5.7951 & 1 & 10 & 5.4402 & 5 & 44 & 23.6942 \\
\hline B32 & 4 & 4 & 4.1935 & 0.7492 & 0.00 & 153 & 159 & 3.6603 & 2 & 7 & 4.7390 & 8 & 31 & 20.0530 \\
\hline B33 & 4 & 4 & 4.0645 & 0.3592 & 0.00 & 153 & 158 & 3.9906 & 2 & 6 & 3.3872 & 8 & 25 & 13.7612 \\
\hline B34 & 4 & 4 & 4.2581 & 0.6816 & 0.00 & 152 & 158 & 3.7035 & 2 & 5 & 3.5636 & 8 & 25 & 14.7240 \\
\hline B35 & 4 & 4 & 4.4516 & 0.8500 & 0.00 & 153 & 158 & 4.3965 & 2 & 5 & 3.3916 & 8 & 22 & 14.3879 \\
\hline B36 & 13 & 13 & 13.0000 & 0.0000 & 0.00 & 156 & 166 & 6.3017 & 1 & 10 & 5.6101 & 5 & 44 & 24.9727 \\
\hline B37 & 10 & 10 & 10.3226 & 0.7478 & 0.00 & 154 & 157 & 2.4314 & 2 & 8 & 6.6553 & 8 & 34 & 27.1586 \\
\hline B38 & 8 & 8 & 8.1613 & 0.4544 & 0.00 & 152 & 156 & 4.2246 & 3 & 10 & 8.3402 & 12 & 44 & 34.0656 \\
\hline B39 & 5 & 5 & 5.0000 & 0.0000 & 0.00 & 153 & 159 & 11.6618 & 3 & 5 & 2.3363 & 12 & 23 & 9.8435 \\
\hline B40 & 5 & 5 & 5.1935 & 0.6011 & 0.00 & 152 & 160 & 6.3128 & 2 & 5 & 2.0645 & 9 & 25 & 8.9185 \\
\hline B41 & 8 & 8 & 8.1613 & 0.8980 & 0.00 & 154 & 169 & 10.6175 & 1 & 8 & 5.2096 & 4 & 36 & 22.4695 \\
\hline B42 & 8 & 8 & 8.0000 & 0.0000 & 0.00 & 153 & 166 & 10.1747 & 2 & 4 & 1.9438 & 8 & 18 & 8.0620 \\
\hline B43 & 8 & 8 & 8.0000 & 0.0000 & 0.00 & 152 & 165 & 10.7002 & 2 & 5 & 2.1568 & 12 & 24 & 9.2664 \\
\hline B44 & 5 & 5 & 5.7742 & 1.3344 & 0.00 & 153 & 161 & 8.5650 & 2 & 4 & 1.8062 & 8 & 21 & 7.8376 \\
\hline B45 & 5 & 5 & 5.9677 & 1.4256 & 0.00 & 152 & 159 & 8.0203 & 2 & 5 & 2.6453 & 8 & 24 & 10.7374 \\
\hline B46 & 8 & 8 & 8.0645 & 0.2497 & 0.00 & 155 & 172 & 12.5416 & 2 & 9 & 6.5325 & 8 & 44 & 31.3379 \\
\hline B47 & 5 & 5 & 5.0968 & 0.5388 & 0.00 & 153 & 162 & 6.1438 & 2 & 6 & 2.2423 & 8 & 26 & 9.7972 \\
\hline B48 & 5 & 5 & 5.1290 & 0.4995 & 0.00 & 152 & 157 & 3.8888 & 2 & 5 & 2.3646 & 8 & 23 & 9.8283 \\
\hline B49 & 5 & 5 & 5.2581 & 0.6816 & 0.00 & 153 & 166 & 11.7214 & 3 & 5 & 2.3335 & 14 & 25 & 9.2466 \\
\hline B50 & 5 & 5 & 5.3226 & 0.7478 & 0.00 & 158 & 297 & 112.2328 & 2 & 5 & 4.2598 & 8 & 46 & 59.0964 \\
\hline
\end{tabular}


TABle 23: Continued.

\begin{tabular}{|c|c|c|c|c|c|c|c|c|c|c|c|c|c|c|}
\hline \multirow{2}{*}{ ID } & \multirow{2}{*}{ OPT } & \multicolumn{4}{|c|}{ Optimum } & \multicolumn{3}{|c|}{ Execution time } & \multicolumn{3}{|c|}{ Cycle } & \multicolumn{3}{|c|}{ Time cycle } \\
\hline & & $\mathrm{MBO}$ & AVG & SD & RPD\% & BET & AVG & ST & $\mathrm{BC}$ & AVG & SD & BTC & AVG & SD \\
\hline B51 & 27 & 27 & 28.1935 & 1.0139 & 0.00 & 170 & 210 & 50.5103 & 9 & 21 & 8.4341 & 40 & 120 & 65.5098 \\
\hline B52 & 18 & 18 & 19.0000 & 1.2383 & 0.00 & 160 & 174 & 12.9624 & 7 & 21 & 8.9031 & 29 & 96 & 39.5778 \\
\hline B53 & 11 & 11 & 11.0000 & 0.0000 & 0.00 & 161 & 178 & 16.1011 & 4 & 10 & 3.4251 & 17 & 49 & 15.4218 \\
\hline B54 & 11 & 11 & 11.0000 & 0.0000 & 0.00 & 164 & 225 & 110.0060 & 4 & 9 & 2.2274 & 17 & 53 & 25.5038 \\
\hline B55 & 7 & 7 & 7.0000 & 0.0000 & 0.00 & 170 & 227 & 95.8822 & 6 & 12 & 4.8697 & 35 & 73 & 34.6457 \\
\hline B56 & 6 & 6 & 6.0000 & 0.0000 & 0.00 & 158 & 176 & 22.7563 & 4 & 10 & 4.1773 & 18 & 46 & 17.2158 \\
\hline B57 & 6 & 6 & 6.0323 & 0.1796 & 0.00 & 158 & 164 & 3.8771 & 6 & 11 & 4.7317 & 29 & 49 & 20.2836 \\
\hline B58 & 6 & 6 & 6.0000 & 0.0000 & 0.00 & 157 & 167 & 7.3482 & 4 & 10 & 4.1721 & 17 & 47 & 17.6931 \\
\hline B59 & 9 & 9 & 9.1290 & 0.3408 & 0.00 & 162 & 176 & 9.1676 & 4 & 12 & 7.6146 & 16 & 60 & 35.3615 \\
\hline B 60 & 4 & 4 & 4.2581 & 0.9989 & 0.00 & 160 & 167 & 5.9831 & 3 & 13 & 7.2062 & 14 & 62 & 31.0985 \\
\hline B61 & 4 & 4 & 4.1290 & 0.3408 & 0.00 & 160 & 165 & 3.8543 & 6 & 12 & 6.4014 & 25 & 53 & 28.3432 \\
\hline B62 & 4 & 4 & 4.0000 & 0.0000 & 0.00 & 158 & 164 & 4.8012 & 4 & 10 & 5.3501 & 18 & 43 & 23.0980 \\
\hline B63 & 27 & 27 & 27.2903 & 0.4614 & 0.00 & 167 & 175 & 6.0466 & 2 & 16 & 7.7237 & 9 & 76 & 36.5377 \\
\hline B64 & 18 & 18 & 18.3871 & 1.0223 & 0.00 & 160 & 168 & 5.1665 & 3 & 19 & 9.7662 & 15 & 86 & 43.3281 \\
\hline B65 & 14 & 14 & 14.0323 & 0.1796 & 0.00 & 160 & 165 & 3.8291 & 4 & 16 & 8.6324 & 17 & 71 & 37.8486 \\
\hline B66 & 13 & 13 & 13.0000 & 0.0000 & 0.00 & 158 & 164 & 4.3960 & 4 & 9 & 3.8069 & 17 & 42 & 17.3308 \\
\hline B67 & 11 & 11 & 11.0000 & 0.0000 & 0.00 & 161 & 169 & 5.7328 & 3 & 13 & 6.7766 & 15 & 60 & 30.8939 \\
\hline B68 & 8 & 8 & 8.5161 & 1.3873 & 0.00 & 157 & 166 & 4.9242 & 5 & 12 & 5.9210 & 20 & 55 & 25.9415 \\
\hline B69 & 8 & 8 & 8.7097 & 0.8244 & 0.00 & 159 & 186 & 80.6533 & 4 & 10 & 3.5186 & 20 & 59 & 78.6617 \\
\hline B70 & 6 & 6 & 6.3548 & 0.6082 & 0.00 & 157 & 164 & 4.1299 & 5 & 11 & 6.0651 & 21 & 49 & 26.3690 \\
\hline B71 & 6 & 6 & 6.3226 & 0.9087 & 0.00 & 164 & 173 & 4.9813 & 3 & 13 & 7.2318 & 15 & 64 & 34.0172 \\
\hline B72 & 4 & 4 & 4.2581 & 0.5143 & 0.00 & 162 & 169 & 7.0074 & 4 & 13 & 7.3266 & 18 & 61 & 31.5165 \\
\hline B73 & 4 & 4 & 4.3548 & 0.5507 & 0.00 & 155 & 163 & 7.4271 & 4 & 10 & 4.5181 & 16 & 46 & 19.8506 \\
\hline B74 & 3 & 3 & 3.3871 & 0.6152 & 0.00 & 154 & 159 & 6.2096 & 4 & 12 & 6.5673 & 18 & 54 & 27.7339 \\
\hline B75 & 11 & 11 & 11.8710 & 0.9217 & 0.00 & 156 & 166 & 6.2745 & 7 & 19 & 9.0094 & 32 & 84 & 39.6957 \\
\hline B76 & 5 & 5 & 5.1290 & 0.4995 & 0.00 & 154 & 159 & 4.0391 & 3 & 12 & 7.1859 & 12 & 53 & 30.5339 \\
\hline B77 & 5 & 5 & 5.6129 & 0.9892 & 0.00 & 154 & 158 & 2.9061 & 3 & 12 & 6.2763 & 12 & 52 & 26.0995 \\
\hline B78 & 4 & 4 & 4.7097 & 1.1887 & 0.00 & 152 & 157 & 4.4870 & 3 & 15 & 8.9251 & 12 & 64 & 37.0953 \\
\hline B79 & 14 & 14 & 14.0000 & 0.0000 & 0.00 & 154 & 160 & 3.9374 & 4 & 10 & 3.9070 & 17 & 46 & 16.9129 \\
\hline B80 & 11 & 11 & 11.0000 & 0.0000 & 0.00 & 154 & 159 & 3.6503 & 4 & 10 & 3.0409 & 16 & 43 & 12.6801 \\
\hline B81 & 11 & 11 & 11.2258 & 0.7169 & 0.00 & 154 & 157 & 2.5025 & 3 & 12 & 7.2826 & 13 & 51 & 29.8398 \\
\hline B82 & 10 & 10 & 10.7097 & 0.7829 & 0.00 & 153 & 160 & 9.2766 & 4 & 10 & 4.2187 & 19 & 45 & 17.2205 \\
\hline B83 & 12 & 12 & 12.1290 & 0.7184 & 0.00 & 154 & 161 & 5.0024 & 5 & 13 & 7.6357 & 23 & 59 & 32.3602 \\
\hline B84 & 12 & 12 & 12.0968 & 0.5388 & 0.00 & 154 & 159 & 3.8746 & 3 & 11 & 5.8103 & 12 & 49 & 25.2224 \\
\hline B85 & 8 & 8 & 9.3548 & 1.4731 & 0.00 & 153 & 157 & 3.7096 & 4 & 13 & 7.1319 & 19 & 57 & 29.5541 \\
\hline B86 & 8 & 8 & 8.0000 & 0.0000 & 0.00 & 152 & 157 & 4.4661 & 4 & 11 & 4.7817 & 16 & 46 & 19.8128 \\
\hline B87 & 10 & 10 & 10.3871 & 0.9193 & 0.00 & 156 & 165 & 5.2583 & 6 & 18 & 8.5468 & 25 & 81 & 38.3296 \\
\hline B88 & 8 & 8 & 8.0000 & 0.0000 & 0.00 & 154 & 159 & 6.6066 & 4 & 11 & 3.8846 & 16 & 47 & 16.1636 \\
\hline B89 & 8 & 8 & 8.0323 & 0.1796 & 0.00 & 153 & 158 & 4.5929 & 3 & 10 & 5.0891 & 12 & 42 & 21.2703 \\
\hline \multirow[t]{2}{*}{ B90 } & 5 & 5 & 5.7742 & 1.3344 & 0.00 & 153 & 157 & 2.6204 & 6 & 11 & 3.8401 & 24 & 46 & 15.4224 \\
\hline & & AVG & 8.1065 & 0.4446 & 0.00 & 155 & 168 & 13.9276 & 3 & 9 & 4.7812 & 13 & 42 & 22.9180 \\
\hline
\end{tabular}


TABLE 24: MBO using parallel merge sort algorithm with 4 threads.

\begin{tabular}{|c|c|c|c|c|c|c|c|c|c|c|c|c|c|c|}
\hline \multirow{2}{*}{ ID } & \multirow{2}{*}{ OPT } & \multicolumn{4}{|c|}{ Optimum } & \multicolumn{3}{|c|}{ Execution time } & \multicolumn{3}{|c|}{ Cycle } & \multicolumn{3}{|c|}{ Time cycle } \\
\hline & & $\mathrm{MBO}$ & AVG & $\mathrm{SD}$ & RPD\% & BET & AVG & ST & $\mathrm{BC}$ & AVG & $\mathrm{SD}$ & BTC & AVG & SD \\
\hline B1 & 11 & 11 & 11.0000 & 0.0000 & 0.00 & 158 & 216 & 109.1743 & 2 & 6 & 3.6142 & 9 & 42 & 25.9406 \\
\hline B2 & 11 & 11 & 11.0000 & 0.0000 & 0.00 & 152 & 157 & 6.6604 & 2 & 4 & 1.2365 & 10 & 18 & 7.4686 \\
\hline B3 & 11 & 11 & 11.0000 & 0.0000 & 0.00 & 152 & 166 & 47.4789 & 1 & 4 & 1.2960 & 4 & 18 & 8.4496 \\
\hline B4 & 11 & 11 & 11.0000 & 0.0000 & 0.00 & 152 & 155 & 2.5716 & 1 & 5 & 2.6331 & 4 & 20 & 10.8924 \\
\hline B5 & 11 & 11 & 11.3871 & 1.4984 & 0.00 & 153 & 167 & 45.7047 & 1 & 6 & 3.3085 & 4 & 29 & 18.6757 \\
\hline B6 & 7 & 7 & 7.0000 & 0.0000 & 0.00 & 154 & 162 & 9.6934 & 2 & 9 & 5.7726 & 8 & 40 & 24.3002 \\
\hline B7 & 6 & 6 & 6.0000 & 0.0000 & 0.00 & 154 & 167 & 41.5747 & 3 & 6 & 2.6845 & 12 & 26 & 10.8095 \\
\hline B8 & 4 & 4 & 4.3226 & 0.7478 & 0.00 & 153 & 155 & 2.5003 & 2 & 11 & 9.7807 & 8 & 49 & 39.5824 \\
\hline B9 & 3 & 3 & 3.0645 & 0.2497 & 0.00 & 152 & 168 & 58.7230 & 2 & 6 & 2.9494 & 8 & 27 & 17.1893 \\
\hline $\mathrm{B} 10$ & 3 & 3 & 3.0000 & 0.0000 & 0.00 & 152 & 166 & 41.0109 & 2 & 4 & 1.9355 & 8 & 20 & 9.1206 \\
\hline B11 & 4 & 4 & 4.1290 & 0.3408 & 0.00 & 155 & 160 & 4.3005 & 2 & 10 & 6.9986 & 8 & 43 & 29.6649 \\
\hline B12 & 4 & 4 & 4.0000 & 0.0000 & 0.00 & 153 & 166 & 41.1127 & 1 & 4 & 1.8044 & 4 & 19 & 7.2704 \\
\hline B13 & 4 & 4 & 4.0000 & 0.0000 & 0.00 & 152 & 155 & 2.5059 & 2 & 6 & 4.9324 & 8 & 25 & 20.2386 \\
\hline B14 & 3 & 3 & 3.1935 & 0.4016 & 0.00 & 152 & 167 & 46.3310 & 1 & 5 & 2.2269 & 4 & 26 & 14.4843 \\
\hline B15 & 1 & 1 & 1.3548 & 0.8386 & 0.00 & 152 & 154 & 2.6249 & 2 & 5 & 3.1356 & 8 & 24 & 12.8711 \\
\hline B16 & 14 & 14 & 14.0000 & 0.0000 & 0.00 & 154 & 171 & 46.8582 & 3 & 8 & 4.1048 & 12 & 36 & 19.3441 \\
\hline B17 & 13 & 13 & 13.0000 & 0.0000 & 0.00 & 154 & 157 & 4.7342 & 2 & 5 & 1.9903 & 8 & 21 & 8.5249 \\
\hline B18 & 13 & 13 & 13.0000 & 0.0000 & 0.00 & 152 & 166 & 40.2106 & 3 & 5 & 1.9210 & 12 & 26 & 11.5105 \\
\hline B19 & 13 & 13 & 13.1935 & 0.7492 & 0.00 & 152 & 157 & 9.6044 & 2 & 7 & 4.3160 & 8 & 29 & 17.8754 \\
\hline B20 & 13 & 13 & 13.0000 & 0.0000 & 0.00 & 152 & 165 & 54.4453 & 2 & 7 & 3.8504 & 8 & 32 & 17.1361 \\
\hline B21 & 9 & 9 & 9.2258 & 0.4250 & 0.00 & 154 & 173 & 54.5359 & 2 & 11 & 7.4947 & 10 & 51 & 32.7887 \\
\hline B22 & 6 & 6 & 6.0968 & 0.3005 & 0.00 & 154 & 160 & 5.8877 & 3 & 5 & 2.8044 & 12 & 24 & 11.6705 \\
\hline B23 & 6 & 6 & 6.0645 & 0.2497 & 0.00 & 152 & 169 & 48.5055 & 2 & 7 & 4.5711 & 8 & 35 & 21.3986 \\
\hline B24 & 5 & 5 & 5.2581 & 0.5755 & 0.00 & 152 & 154 & 2.3010 & 2 & 6 & 2.7875 & 8 & 25 & 11.3416 \\
\hline B25 & 4 & 4 & 4.1935 & 0.4016 & 0.00 & 152 & 167 & 46.4296 & 2 & 6 & 2.6644 & 8 & 27 & 23.6771 \\
\hline B26 & 5 & 5 & 5.0000 & 0.0000 & 0.00 & 154 & 158 & 3.7574 & 3 & 8 & 6.3478 & 15 & 37 & 26.7206 \\
\hline B27 & 3 & 3 & 3.0323 & 0.1796 & 0.00 & 154 & 168 & 47.1433 & 2 & 5 & 3.5903 & 9 & 26 & 19.3762 \\
\hline B28 & 3 & 3 & 3.3226 & 0.4752 & 0.00 & 152 & 158 & 19.6735 & 1 & 5 & 3.6966 & 4 & 23 & 15.3801 \\
\hline B29 & 3 & 3 & 3.1613 & 0.4544 & 0.00 & 152 & 163 & 41.5225 & 2 & 5 & 3.3315 & 8 & 25 & 35.7845 \\
\hline B30 & 2 & 2 & 2.4839 & 0.5699 & 0.00 & 152 & 165 & 44.6843 & 2 & 7 & 5.1800 & 8 & 30 & 22.1249 \\
\hline B31 & 7 & 7 & 7.0000 & 0.0000 & 0.00 & 153 & 161 & 6.9997 & 3 & 8 & 4.2651 & 12 & 37 & 18.4910 \\
\hline B32 & 4 & 4 & 4.2903 & 0.9016 & 0.00 & 155 & 205 & 147.1775 & 1 & 8 & 5.0298 & 5 & 40 & 29.8585 \\
\hline B33 & 4 & 4 & 4.0645 & 0.3592 & 0.00 & 153 & 172 & 25.2682 & 2 & 5 & 3.2974 & 8 & 25 & 13.9995 \\
\hline B34 & 4 & 4 & 4.3226 & 0.7478 & 0.00 & 152 & 155 & 3.4554 & 1 & 6 & 6.1399 & 4 & 28 & 24.5491 \\
\hline B35 & 4 & 4 & 4.3871 & 0.8032 & 0.00 & 152 & 157 & 7.2196 & 2 & 5 & 2.8979 & 8 & 23 & 11.7220 \\
\hline B36 & 13 & 13 & 13.0000 & 0.0000 & 0.00 & 158 & 172 & 15.0594 & 3 & 10 & 5.7384 & 12 & 46 & 26.7314 \\
\hline B37 & 10 & 10 & 10.4516 & 0.8500 & 0.00 & 154 & 166 & 14.1400 & 2 & 8 & 6.8311 & 8 & 38 & 28.5876 \\
\hline B38 & 8 & 8 & 8.0323 & 0.1796 & 0.00 & 155 & 164 & 12.4326 & 2 & 8 & 5.6068 & 8 & 37 & 23.8998 \\
\hline B39 & 5 & 5 & 5.3548 & 1.3796 & 0.00 & 154 & 163 & 12.5069 & 2 & 5 & 2.0884 & 8 & 22 & 9.4440 \\
\hline B40 & 5 & 5 & 5.2581 & 0.6816 & 0.00 & 153 & 165 & 16.5927 & 2 & 5 & 3.6106 & 8 & 24 & 15.9870 \\
\hline B41 & 8 & 8 & 8.1613 & 0.8980 & 0.00 & 156 & 166 & 9.9435 & 1 & 10 & 8.3483 & 4 & 47 & 36.1707 \\
\hline B42 & 8 & 8 & 8.0000 & 0.0000 & 0.00 & 155 & 174 & 34.2187 & 2 & 4 & 1.6350 & 8 & 19 & 8.1873 \\
\hline B43 & 8 & 8 & 8.0000 & 0.0000 & 0.00 & 153 & 198 & 46.1624 & 1 & 4 & 2.1423 & 4 & 24 & 13.0368 \\
\hline B44 & 5 & 5 & 5.7742 & 1.3344 & 0.00 & 156 & 183 & 32.3323 & 2 & 5 & 1.6919 & 10 & 25 & 9.0367 \\
\hline B45 & 5 & 5 & 5.8065 & 1.4005 & 0.00 & 152 & 157 & 4.5948 & 2 & 5 & 2.8658 & 9 & 24 & 12.1692 \\
\hline B46 & 8 & 8 & 8.0323 & 0.1796 & 0.00 & 157 & 176 & 28.1980 & 3 & 10 & 7.1360 & 14 & 50 & 40.1945 \\
\hline B47 & 5 & 5 & 5.1935 & 0.7492 & 0.00 & 155 & 183 & 36.2397 & 2 & 6 & 2.5649 & 8 & 31 & 16.0299 \\
\hline B48 & 5 & 5 & 5.1935 & 0.6011 & 0.00 & 152 & 166 & 23.6329 & 2 & 5 & 2.2832 & 8 & 24 & 10.1954 \\
\hline B49 & 5 & 5 & 5.2581 & 0.6816 & 0.00 & 152 & 155 & 2.8071 & 2 & 5 & 2.4613 & 8 & 22 & 10.1229 \\
\hline B50 & 5 & 5 & 5.1290 & 0.4995 & 0.00 & 152 & 155 & 4.6881 & 2 & 7 & 5.3336 & 8 & 30 & 25.5601 \\
\hline
\end{tabular}


TABle 24: Continued.

\begin{tabular}{|c|c|c|c|c|c|c|c|c|c|c|c|c|c|c|}
\hline \multirow{2}{*}{ ID } & \multirow{2}{*}{ OPT } & \multicolumn{4}{|c|}{ Optimum } & \multicolumn{3}{|c|}{ Execution time } & \multicolumn{3}{|c|}{ Cycle } & \multicolumn{3}{|c|}{ Time cycle } \\
\hline & & $\mathrm{MBO}$ & AVG & SD & RPD\% & BET & AVG & ST & $\mathrm{BC}$ & AVG & SD & BTC & AVG & SD \\
\hline B51 & 27 & 27 & 28.2903 & 1.0064 & 0.00 & 159 & 164 & 4.3110 & 4 & 22 & 9.2894 & 16 & 98 & 40.3385 \\
\hline B52 & 18 & 18 & 19.0645 & 1.0935 & 0.00 & 154 & 159 & 5.8249 & 5 & 22 & 9.8280 & 22 & 95 & 42.6600 \\
\hline B53 & 11 & 11 & 11.0000 & 0.0000 & 0.00 & 153 & 178 & 32.7949 & 4 & 11 & 3.7102 & 19 & 51 & 18.7233 \\
\hline B54 & 11 & 11 & 11.0000 & 0.0000 & 0.00 & 153 & 164 & 19.9278 & 3 & 7 & 2.7140 & 12 & 34 & 14.0533 \\
\hline B55 & 7 & 7 & 7.0000 & 0.0000 & 0.00 & 155 & 160 & 3.4790 & 2 & 10 & 4.3510 & 8 & 46 & 18.3698 \\
\hline B56 & 6 & 6 & 6.0000 & 0.0000 & 0.00 & 154 & 156 & 1.7121 & 2 & 10 & 3.2772 & 8 & 42 & 13.7300 \\
\hline B57 & 6 & 6 & 6.0323 & 0.1796 & 0.00 & 153 & 160 & 16.4040 & 4 & 10 & 3.8788 & 17 & 45 & 16.0311 \\
\hline B58 & 6 & 6 & 6.0323 & 0.1796 & 0.00 & 154 & 168 & 26.7755 & 3 & 10 & 3.9119 & 12 & 45 & 16.9634 \\
\hline B59 & 9 & 9 & 9.2258 & 0.4973 & 0.00 & 155 & 194 & 38.6829 & 3 & 15 & 8.8444 & 13 & 76 & 44.3162 \\
\hline B60 & 4 & 4 & 4.9032 & 1.7001 & 0.00 & 154 & 169 & 24.2931 & 3 & 12 & 6.6625 & 13 & 54 & 30.0641 \\
\hline B61 & 4 & 4 & 4.1290 & 0.5623 & 0.00 & 155 & 268 & 200.8557 & 4 & 12 & 7.0176 & 16 & 109 & 179.0541 \\
\hline B62 & 4 & 4 & 4.0000 & 0.0000 & 0.00 & 153 & 157 & 4.2149 & 3 & 9 & 3.8691 & 13 & 40 & 15.6801 \\
\hline B63 & 27 & 27 & 27.2581 & 0.5143 & 0.00 & 154 & 165 & 10.6263 & 6 & 16 & 9.2118 & 24 & 71 & 40.8554 \\
\hline B64 & 18 & 18 & 18.4516 & 0.8099 & 0.00 & 154 & 178 & 18.2276 & 7 & 19 & 9.1876 & 31 & 91 & 45.9490 \\
\hline B65 & 14 & 14 & 14.0323 & 0.1796 & 0.00 & 155 & 161 & 7.8149 & 6 & 15 & 7.3228 & 25 & 68 & 30.9394 \\
\hline B66 & 13 & 13 & 13.0000 & 0.0000 & 0.00 & 155 & 194 & 41.5668 & 6 & 10 & 3.4251 & 24 & 52 & 21.8884 \\
\hline B67 & 11 & 11 & 11.0323 & 0.1796 & 0.00 & 158 & 210 & 67.1852 & 1 & 13 & 6.8971 & 4 & 72 & 42.9510 \\
\hline B68 & 8 & 8 & 8.3226 & 1.2751 & 0.00 & 153 & 197 & 51.5259 & 4 & 13 & 7.9708 & 16 & 68 & 52.5572 \\
\hline B69 & 8 & 8 & 8.5806 & 0.6720 & 0.00 & 155 & 208 & 84.9156 & 5 & 11 & 5.6323 & 21 & 60 & 31.5805 \\
\hline B70 & 6 & 6 & 6.5484 & 0.8884 & 0.00 & 154 & 231 & 66.9705 & 4 & 15 & 8.5271 & 29 & 91 & 56.9068 \\
\hline B71 & 6 & 6 & 6.0645 & 0.3592 & 0.00 & 157 & 217 & 70.6903 & 3 & 12 & 6.9808 & 14 & 71 & 46.9560 \\
\hline B72 & 4 & 4 & 4.2258 & 0.4250 & 0.00 & 161 & 189 & 40.5461 & 5 & 13 & 7.1670 & 24 & 68 & 41.0736 \\
\hline B73 & 4 & 4 & 4.4516 & 0.6239 & 0.00 & 156 & 177 & 29.4202 & 4 & 13 & 5.3836 & 20 & 59 & 23.6061 \\
\hline B74 & 3 & 3 & 3.3871 & 0.5584 & 0.00 & 154 & 161 & 4.3200 & 3 & 13 & 7.5998 & 12 & 55 & 32.4734 \\
\hline B75 & 11 & 11 & 11.7742 & 1.0866 & 0.00 & 155 & 172 & 13.9606 & 4 & 15 & 9.4963 & 16 & 69 & 45.7583 \\
\hline B76 & 5 & 5 & 5.1935 & 0.6011 & 0.00 & 154 & 159 & 4.2812 & 4 & 13 & 6.7199 & 16 & 55 & 28.2946 \\
\hline B77 & 5 & 5 & 5.9677 & 1.1968 & 0.00 & 154 & 157 & 3.0589 & 4 & 12 & 7.3398 & 16 & 54 & 30.8010 \\
\hline B78 & 4 & 4 & 4.6452 & 1.1120 & 0.00 & 153 & 156 & 2.6757 & 1 & 13 & 7.3425 & 4 & 56 & 30.7701 \\
\hline B79 & 14 & 14 & 14.0000 & 0.0000 & 0.00 & 154 & 161 & 5.2525 & 4 & 10 & 2.9487 & 18 & 43 & 12.1078 \\
\hline B80 & 11 & 11 & 11.0000 & 0.0000 & 0.00 & 155 & 202 & 147.0668 & 3 & 11 & 5.4449 & 16 & 50 & 26.2353 \\
\hline B81 & 11 & 11 & 11.4516 & 0.9605 & 0.00 & 155 & 208 & 59.8935 & 7 & 13 & 4.7648 & 28 & 74 & 32.8557 \\
\hline B82 & 10 & 10 & 10.6452 & 0.7978 & 0.00 & 155 & 190 & 37.9565 & 4 & 13 & 7.3710 & 20 & 68 & 41.9503 \\
\hline B83 & 12 & 12 & 12.0000 & 0.0000 & 0.00 & 155 & 162 & 6.1394 & 3 & 10 & 5.8394 & 12 & 48 & 28.0464 \\
\hline B84 & 12 & 12 & 12.1935 & 0.6011 & 0.00 & 154 & 158 & 2.8090 & 7 & 12 & 6.3531 & 28 & 53 & 25.9466 \\
\hline B85 & 8 & 8 & 9.1613 & 1.7146 & 0.00 & 154 & 156 & 1.8346 & 4 & 13 & 6.5165 & 16 & 57 & 27.0130 \\
\hline B86 & 8 & 8 & 8.0000 & 0.0000 & 0.00 & 153 & 157 & 3.0107 & 3 & 11 & 3.7543 & 14 & 47 & 15.8781 \\
\hline B87 & 10 & 10 & 10.8065 & 1.0462 & 0.00 & 156 & 168 & 14.7487 & 6 & 17 & 9.3396 & 24 & 78 & 43.8068 \\
\hline B88 & 8 & 8 & 8.1935 & 1.0776 & 0.00 & 154 & 157 & 2.8132 & 4 & 10 & 5.2145 & 16 & 44 & 21.6391 \\
\hline B89 & 8 & 8 & 8.0000 & 0.0000 & 0.00 & 154 & 157 & 2.5607 & 3 & 10 & 4.7956 & 13 & 44 & 20.3743 \\
\hline \multirow[t]{2}{*}{ B90 } & 5 & 5 & 5.3871 & 1.0223 & 0.00 & 154 & 156 & 2.1397 & 4 & 11 & 4.6769 & 16 & 46 & 19.3850 \\
\hline & & AVG & 8.1183 & 0.4847 & 0.00 & 153 & 170 & 28.8802 & 2 & 9 & 4.9501 & 12 & 43 & 25.6019 \\
\hline
\end{tabular}


TABLE 25: MBO using parallel merge sort algorithm with 8 threads.

\begin{tabular}{|c|c|c|c|c|c|c|c|c|c|c|c|c|c|c|}
\hline \multirow{2}{*}{ ID } & \multirow{2}{*}{ OPT } & \multicolumn{4}{|c|}{ Optimum } & \multicolumn{3}{|c|}{ Execution time } & \multicolumn{3}{|c|}{ Cycle } & \multicolumn{3}{|c|}{ Time cycle } \\
\hline & & $\mathrm{MBO}$ & AVG & SD & RPD\% & BET & AVG & ST & $\mathrm{BC}$ & AVG & SD & BTC & AVG & SD \\
\hline B1 & 11 & 11 & 11.0000 & 0.0000 & 0.00 & 153 & 175 & 52.9600 & 2 & 7 & 3.4650 & 10 & 40 & 24.1691 \\
\hline B2 & 11 & 11 & 11.0000 & 0.0000 & 0.00 & 152 & 155 & 4.2538 & 1 & 4 & 1.3663 & 4 & 17 & 6.3123 \\
\hline B3 & 11 & 11 & 11.0000 & 0.0000 & 0.00 & 152 & 154 & 2.2737 & 2 & 4 & 1.2675 & 8 & 16 & 4.9966 \\
\hline B4 & 11 & 11 & 11.0000 & 0.0000 & 0.00 & 152 & 154 & 2.1875 & 3 & 5 & 1.6669 & 12 & 22 & 6.6451 \\
\hline B5 & 11 & 11 & 11.0000 & 0.0000 & 0.00 & 152 & 154 & 3.5484 & 1 & 5 & 4.6460 & 4 & 22 & 19.1976 \\
\hline B6 & 7 & 7 & 7.0000 & 0.0000 & 0.00 & 154 & 159 & 6.9589 & 3 & 9 & 5.6927 & 12 & 41 & 23.3150 \\
\hline B7 & 6 & 6 & 6.0323 & 0.1796 & 0.00 & 152 & 155 & 2.6567 & 2 & 5 & 3.3376 & 8 & 24 & 13.6479 \\
\hline B8 & 4 & 4 & 4.2581 & 0.6816 & 0.00 & 152 & 154 & 2.2500 & 2 & 10 & 7.8339 & 8 & 43 & 31.7022 \\
\hline B9 & 3 & 3 & 3.0645 & 0.2497 & 0.00 & 152 & 154 & 2.8853 & 1 & 6 & 4.2480 & 4 & 26 & 17.5545 \\
\hline $\mathrm{B} 10$ & 3 & 3 & 3.0000 & 0.0000 & 0.00 & 152 & 155 & 4.3342 & 2 & 4 & 2.2017 & 8 & 19 & 9.0625 \\
\hline B11 & 4 & 4 & 4.1613 & 0.3739 & 0.00 & 154 & 158 & 4.0725 & 3 & 11 & 6.8310 & 12 & 46 & 28.0720 \\
\hline B12 & 4 & 4 & 4.0000 & 0.0000 & 0.00 & 152 & 154 & 2.2041 & 1 & 4 & 1.7487 & 4 & 18 & 7.3882 \\
\hline B13 & 4 & 4 & 4.0645 & 0.3592 & 0.00 & 152 & 157 & 8.3824 & 2 & 6 & 4.0825 & 8 & 24 & 16.4007 \\
\hline B14 & 3 & 3 & 3.4516 & 0.5059 & 0.00 & 152 & 156 & 10.8955 & 2 & 5 & 2.0848 & 8 & 21 & 8.3643 \\
\hline B15 & 1 & 1 & 1.2903 & 0.7829 & 0.00 & 152 & 153 & 1.8322 & 2 & 5 & 2.4110 & 8 & 23 & 9.8292 \\
\hline B16 & 14 & 14 & 14.0000 & 0.0000 & 0.00 & 155 & 160 & 4.5349 & 2 & 7 & 3.6044 & 10 & 32 & 14.9542 \\
\hline B17 & 13 & 13 & 13.0000 & 0.0000 & 0.00 & 152 & 155 & 2.2423 & 2 & 4 & 1.6145 & 8 & 20 & 6.7850 \\
\hline B18 & 13 & 13 & 13.0000 & 0.0000 & 0.00 & 152 & 154 & 3.8763 & 1 & 5 & 2.1553 & 4 & 22 & 9.3721 \\
\hline B19 & 13 & 13 & 13.0000 & 0.0000 & 0.00 & 152 & 153 & 1.4796 & 2 & 7 & 6.3034 & 8 & 28 & 25.4949 \\
\hline B20 & 13 & 13 & 13.0000 & 0.0000 & 0.00 & 152 & 153 & 1.4803 & 2 & 8 & 5.1278 & 8 & 33 & 20.5847 \\
\hline B21 & 9 & 9 & 9.1613 & 0.3739 & 0.00 & 154 & 158 & 2.8485 & 2 & 12 & 10.3146 & 8 & 52 & 42.6586 \\
\hline B22 & 6 & 6 & 6.2581 & 0.6308 & 0.00 & 152 & 155 & 3.1380 & 2 & 6 & 2.8459 & 8 & 25 & 11.7755 \\
\hline B23 & 6 & 6 & 6.1935 & 0.5428 & 0.00 & 152 & 153 & 2.0198 & 2 & 10 & 7.3810 & 8 & 43 & 30.1207 \\
\hline B24 & 5 & 5 & 5.2581 & 0.5755 & 0.00 & 152 & 154 & 1.9355 & 1 & 7 & 3.5024 & 4 & 28 & 14.1402 \\
\hline B25 & 4 & 4 & 4.2581 & 0.5143 & 0.00 & 152 & 153 & 2.0562 & 2 & 6 & 3.2132 & 8 & 26 & 12.9135 \\
\hline B26 & 5 & 5 & 5.0000 & 0.0000 & 0.00 & 154 & 158 & 4.3165 & 2 & 9 & 6.1518 & 8 & 39 & 26.6302 \\
\hline B27 & 3 & 3 & 3.0645 & 0.2497 & 0.00 & 152 & 155 & 2.3085 & 2 & 7 & 3.3033 & 8 & 30 & 13.5624 \\
\hline B28 & 3 & 3 & 3.2581 & 0.5755 & 0.00 & 152 & 153 & 1.9680 & 2 & 5 & 3.0261 & 8 & 24 & 13.0409 \\
\hline B29 & 3 & 3 & 3.2258 & 0.4250 & 0.00 & 152 & 154 & 2.4705 & 2 & 4 & 1.8228 & 8 & 18 & 7.6122 \\
\hline B30 & 2 & 2 & 2.5484 & 0.6239 & 0.00 & 152 & 154 & 3.7018 & 2 & 7 & 5.7358 & 8 & 28 & 23.3637 \\
\hline B31 & 7 & 7 & 7.0000 & 0.0000 & 0.00 & 154 & 158 & 3.0953 & 2 & 10 & 6.2340 & 8 & 42 & 25.8763 \\
\hline B32 & 4 & 4 & 4.3871 & 1.2826 & 0.00 & 152 & 155 & 2.7875 & 2 & 8 & 5.7081 & 8 & 36 & 23.1095 \\
\hline B33 & 4 & 4 & 4.1935 & 0.6011 & 0.00 & 152 & 154 & 3.7125 & 2 & 5 & 3.5753 & 8 & 21 & 15.1291 \\
\hline B34 & 4 & 4 & 4.1290 & 0.4995 & 0.00 & 152 & 153 & 1.5268 & 2 & 5 & 4.2205 & 8 & 23 & 16.8363 \\
\hline B35 & 4 & 4 & 4.6452 & 0.9504 & 0.00 & 152 & 152 & 1.0984 & 1 & 5 & 2.2177 & 4 & 21 & 8.8371 \\
\hline B36 & 13 & 13 & 13.0000 & 0.0000 & 0.00 & 154 & 158 & 3.6041 & 4 & 9 & 4.6647 & 16 & 39 & 19.2969 \\
\hline B37 & 10 & 10 & 10.3871 & 0.8032 & 0.00 & 152 & 155 & 4.3569 & 1 & 6 & 2.7689 & 4 & 24 & 11.4273 \\
\hline B38 & 8 & 8 & 8.2258 & 0.4973 & 0.00 & 152 & 154 & 2.2026 & 2 & 9 & 7.5453 & 8 & 40 & 30.0411 \\
\hline B39 & 5 & 5 & 5.0000 & 0.0000 & 0.00 & 152 & 154 & 2.2327 & 2 & 5 & 2.9759 & 9 & 22 & 11.9398 \\
\hline B40 & 5 & 5 & 5.1935 & 0.6011 & 0.00 & 152 & 153 & 1.9989 & 2 & 5 & 2.7552 & 8 & 22 & 11.5980 \\
\hline B41 & 8 & 8 & 8.0000 & 0.0000 & 0.00 & 154 & 159 & 4.8193 & 3 & 10 & 5.9482 & 14 & 45 & 24.6265 \\
\hline B42 & 8 & 8 & 8.0000 & 0.0000 & 0.00 & 152 & 154 & 2.3692 & 2 & 4 & 2.3592 & 8 & 20 & 9.9095 \\
\hline B43 & 8 & 8 & 8.0645 & 0.3592 & 0.00 & 152 & 154 & 1.8526 & 1 & 5 & 2.9563 & 4 & 21 & 12.2532 \\
\hline B44 & 5 & 5 & 6.3548 & 1.5176 & 0.00 & 152 & 153 & 1.1538 & 2 & 5 & 2.9494 & 8 & 24 & 11.9644 \\
\hline B45 & 5 & 5 & 6.1613 & 1.4854 & 0.00 & 152 & 153 & 1.5443 & 2 & 5 & 2.6917 & 8 & 23 & 10.8397 \\
\hline B46 & 8 & 8 & 8.0323 & 0.1796 & 0.00 & 155 & 159 & 4.2805 & 2 & 9 & 6.9125 & 8 & 41 & 28.3549 \\
\hline B47 & 5 & 5 & 5.0968 & 0.5388 & 0.00 & 152 & 154 & 2.0294 & 2 & 5 & 2.0811 & 8 & 21 & 8.4729 \\
\hline B48 & 5 & 5 & 5.1935 & 0.6011 & 0.00 & 152 & 154 & 1.6428 & 2 & 4 & 2.2647 & 8 & 20 & 9.1640 \\
\hline B49 & 5 & 5 & 5.2581 & 0.6816 & 0.00 & 151 & 153 & 3.5342 & 2 & 5 & 2.6482 & 8 & 21 & 10.6357 \\
\hline B50 & 5 & 5 & 5.1290 & 0.4995 & 0.00 & 152 & 153 & 3.1826 & 2 & 5 & 2.8493 & 8 & 22 & 11.5451 \\
\hline
\end{tabular}


TABle 25: Continued.

\begin{tabular}{|c|c|c|c|c|c|c|c|c|c|c|c|c|c|c|}
\hline \multirow{2}{*}{ ID } & \multirow{2}{*}{ OPT } & \multicolumn{4}{|c|}{ Optimum } & \multicolumn{3}{|c|}{ Execution time } & \multicolumn{3}{|c|}{ Cycle } & \multicolumn{3}{|c|}{ Time cycle } \\
\hline & & $\mathrm{MBO}$ & AVG & $\mathrm{SD}$ & RPD\% & BET & AVG & ST & $\mathrm{BC}$ & AVG & $\mathrm{SD}$ & BTC & AVG & $\mathrm{SD}$ \\
\hline B51 & 27 & 27 & 27.9355 & 0.8139 & 0.00 & 154 & 161 & 3.4660 & 10 & 26 & 8.4370 & 41 & 111 & 35.6271 \\
\hline B52 & 18 & 18 & 18.9355 & 1.0626 & 0.00 & 154 & 157 & 3.3758 & 8 & 24 & 9.0310 & 32 & 102 & 36.6151 \\
\hline B53 & 11 & 11 & 11.0000 & 0.0000 & 0.00 & 153 & 155 & 1.8334 & 6 & 11 & 4.6911 & 24 & 45 & 19.0585 \\
\hline B54 & 11 & 11 & 11.0000 & 0.0000 & 0.00 & 152 & 154 & 3.7637 & 3 & 8 & 2.6941 & 12 & 35 & 11.1208 \\
\hline B55 & 7 & 7 & 7.0000 & 0.0000 & 0.00 & 154 & 158 & 3.2984 & 3 & 11 & 5.1034 & 13 & 49 & 21.0208 \\
\hline B56 & 6 & 6 & 6.0000 & 0.0000 & 0.00 & 153 & 156 & 3.0093 & 4 & 11 & 3.8401 & 16 & 48 & 15.2743 \\
\hline B57 & 6 & 6 & 6.1290 & 0.4275 & 0.00 & 153 & 155 & 2.8387 & 4 & 11 & 5.4081 & 16 & 46 & 22.2407 \\
\hline B58 & 6 & 6 & 6.0645 & 0.2497 & 0.00 & 152 & 155 & 4.2566 & 3 & 10 & 3.8702 & 12 & 43 & 15.8509 \\
\hline B59 & 9 & 9 & 9.0323 & 0.1796 & 0.00 & 155 & 158 & 3.0526 & 5 & 14 & 6.9780 & 22 & 60 & 29.3752 \\
\hline B60 & 4 & 4 & 4.3871 & 1.2021 & 0.00 & 153 & 156 & 2.1687 & 5 & 14 & 7.0227 & 21 & 59 & 29.2113 \\
\hline B61 & 4 & 4 & 4.1613 & 0.4544 & 0.00 & 153 & 156 & 4.8604 & 3 & 12 & 6.3903 & 12 & 52 & 27.7761 \\
\hline B62 & 4 & 4 & 4.0323 & 0.1796 & 0.00 & 152 & 154 & 2.3678 & 4 & 10 & 4.8644 & 17 & 45 & 20.3279 \\
\hline B63 & 27 & 27 & 27.1935 & 0.4016 & 0.00 & 156 & 162 & 4.4719 & 4 & 15 & 7.7450 & 16 & 66 & 32.7914 \\
\hline B64 & 18 & 18 & 18.0968 & 0.3005 & 0.00 & 154 & 157 & 2.4722 & 5 & 19 & 8.3824 & 23 & 81 & 35.1227 \\
\hline B65 & 14 & 14 & 14.0323 & 0.1796 & 0.00 & 153 & 156 & 4.1228 & 6 & 15 & 8.5828 & 24 & 65 & 35.2348 \\
\hline B66 & 13 & 13 & 13.0000 & 0.0000 & 0.00 & 152 & 155 & 2.5179 & 7 & 9 & 2.7072 & 28 & 41 & 11.3806 \\
\hline B67 & 11 & 11 & 11.1290 & 0.3408 & 0.00 & 154 & 159 & 4.6810 & 4 & 12 & 6.4386 & 17 & 52 & 27.4067 \\
\hline B68 & 8 & 8 & 8.2258 & 0.9205 & 0.00 & 154 & 157 & 3.4830 & 3 & 14 & 9.5942 & 12 & 61 & 40.0795 \\
\hline B69 & 8 & 8 & 8.6129 & 0.7154 & 0.00 & 153 & 156 & 4.5924 & 4 & 11 & 5.9648 & 16 & 48 & 24.0260 \\
\hline B70 & 6 & 6 & 6.3548 & 0.7549 & 0.00 & 152 & 159 & 9.1831 & 3 & 14 & 6.6604 & 12 & 60 & 29.2535 \\
\hline B71 & 6 & 6 & 6.0000 & 0.0000 & 0.00 & 154 & 160 & 5.0885 & 6 & 14 & 6.2436 & 28 & 60 & 26.1248 \\
\hline B72 & 4 & 4 & 4.2581 & 0.5143 & 0.00 & 153 & 156 & 2.0956 & 3 & 11 & 5.6317 & 13 & 49 & 23.2173 \\
\hline B73 & 4 & 4 & 4.3548 & 0.6607 & 0.00 & 152 & 155 & 4.3015 & 3 & 12 & 7.6978 & 12 & 51 & 31.3144 \\
\hline B74 & 3 & 3 & 3.4839 & 0.5699 & 0.00 & 152 & 154 & 2.1038 & 3 & 11 & 6.2472 & 12 & 45 & 25.2912 \\
\hline B75 & 11 & 11 & 11.7419 & 0.9298 & 0.00 & 154 & 160 & 4.5345 & 3 & 17 & 8.7525 & 13 & 72 & 37.3021 \\
\hline B76 & 5 & 5 & 5.0645 & 0.3592 & 0.00 & 153 & 156 & 2.3192 & 4 & 12 & 6.1097 & 19 & 53 & 25.0673 \\
\hline B77 & 5 & 5 & 5.5161 & 0.8896 & 0.00 & 152 & 155 & 4.3891 & 3 & 12 & 7.1977 & 12 & 52 & 29.2321 \\
\hline B78 & 4 & 4 & 4.6774 & 1.1072 & 0.00 & 152 & 155 & 2.3908 & 4 & 15 & 6.2862 & 17 & 62 & 25.6253 \\
\hline B79 & 14 & 14 & 14.0000 & 0.0000 & 0.00 & 155 & 159 & 3.0324 & 6 & 10 & 4.4923 & 24 & 43 & 18.5246 \\
\hline B80 & 11 & 11 & 11.0000 & 0.0000 & 0.00 & 153 & 157 & 3.3665 & 4 & 11 & 3.6697 & 16 & 46 & 15.4853 \\
\hline B81 & 11 & 11 & 11.3871 & 0.8032 & 0.00 & 152 & 156 & 4.2659 & 3 & 14 & 8.3982 & 12 & 61 & 34.5279 \\
\hline B82 & 10 & 10 & 10.6774 & 0.7911 & 0.00 & 153 & 155 & 1.8613 & 5 & 12 & 5.4156 & 21 & 53 & 22.2498 \\
\hline B83 & 12 & 12 & 12.1935 & 0.7492 & 0.00 & 154 & 160 & 4.3436 & 2 & 11 & 5.9686 & 8 & 50 & 25.0351 \\
\hline B84 & 12 & 12 & 12.1290 & 0.4995 & 0.00 & 153 & 156 & 2.6567 & 6 & 13 & 6.1890 & 25 & 55 & 25.4035 \\
\hline B85 & 8 & 8 & 9.5806 & 1.8934 & 0.00 & 153 & 156 & 3.8652 & 3 & 13 & 6.6922 & 13 & 55 & 27.3698 \\
\hline B86 & 8 & 8 & 8.0000 & 0.0000 & 0.00 & 152 & 155 & 2.2504 & 4 & 10 & 4.1195 & 16 & 43 & 17.4584 \\
\hline B87 & 10 & 10 & 10.4839 & 0.9263 & 0.00 & 155 & 159 & 3.6559 & 4 & 18 & 8.6221 & 16 & 79 & 36.8990 \\
\hline B88 & 8 & 8 & 8.0000 & 0.0000 & 0.00 & 154 & 156 & 2.8530 & 4 & 10 & 3.8077 & 16 & 41 & 15.5335 \\
\hline B89 & 8 & 8 & 8.0000 & 0.0000 & 0.00 & 152 & 156 & 4.3232 & 3 & 9 & 3.6948 & 12 & 39 & 15.6479 \\
\hline \multirow[t]{2}{*}{ B90 } & 5 & 5 & 5.3871 & 1.0223 & 0.00 & 152 & 155 & 3.4501 & 3 & 11 & 4.1108 & 13 & 45 & 16.8234 \\
\hline & & AVG & 8.1029 & 0.4293 & 0.00 & 152 & 155 & 3.8451 & 2 & 9 & 4.8785 & 12 & 40 & 20.2717 \\
\hline
\end{tabular}


TABLE 26: MBO using parallel merge sort algorithm with 16 threads.

\begin{tabular}{|c|c|c|c|c|c|c|c|c|c|c|c|c|c|c|}
\hline \multirow{2}{*}{ ID } & \multirow{2}{*}{ OPT } & \multicolumn{4}{|c|}{ Optimum } & \multicolumn{3}{|c|}{ Execution time } & \multicolumn{3}{|c|}{ Cycle } & \multicolumn{3}{|c|}{ Time cycle } \\
\hline & & $\mathrm{MBO}$ & AVG & SD & RPD\% & BET & AVG & ST & $\mathrm{BC}$ & AVG & $\mathrm{SD}$ & BTC & AVG & $\mathrm{SD}$ \\
\hline B1 & 11 & 11 & 11.0000 & 0.0000 & 0.00 & 156 & 174 & 38.3904 & 1 & 6 & 3.8370 & 4 & 33 & 26.3519 \\
\hline B2 & 11 & 11 & 11.0000 & 0.0000 & 0.00 & 152 & 170 & 37.5675 & 2 & 4 & 1.4338 & 8 & 21 & 9.5525 \\
\hline B3 & 11 & 11 & 11.0000 & 0.0000 & 0.00 & 152 & 153 & 1.4421 & 2 & 4 & 1.3384 & 8 & 18 & 5.5949 \\
\hline B4 & 11 & 11 & 11.0000 & 0.0000 & 0.00 & 152 & 184 & 126.1403 & 3 & 5 & 1.9620 & 12 & 25 & 9.3102 \\
\hline B5 & 11 & 11 & 11.0000 & 0.0000 & 0.00 & 153 & 176 & 53.4639 & 1 & 5 & 2.5716 & 4 & 25 & 14.3306 \\
\hline B6 & 7 & 7 & 7.0000 & 0.0000 & 0.00 & 154 & 170 & 28.7805 & 3 & 10 & 4.5744 & 12 & 47 & 22.7679 \\
\hline B7 & 6 & 6 & 6.0323 & 0.1796 & 0.00 & 155 & 178 & 33.1697 & 3 & 5 & 3.8685 & 12 & 28 & 17.8745 \\
\hline B8 & 4 & 4 & 4.3871 & 0.8032 & 0.00 & 152 & 177 & 28.8102 & 2 & 12 & 10.4629 & 9 & 57 & 49.2948 \\
\hline B9 & 3 & 3 & 3.1290 & 0.3408 & 0.00 & 154 & 177 & 40.8947 & 2 & 6 & 6.0373 & 10 & 33 & 28.3257 \\
\hline $\mathrm{B} 10$ & 3 & 3 & 3.0000 & 0.0000 & 0.00 & 154 & 200 & 42.9216 & 3 & 4 & 1.3125 & 12 & 24 & 8.8010 \\
\hline B11 & 4 & 4 & 4.2258 & 0.4250 & 0.00 & 154 & 208 & 57.8835 & 2 & 11 & 7.2915 & 9 & 61 & 47.9815 \\
\hline B12 & 4 & 4 & 4.0000 & 0.0000 & 0.00 & 154 & 161 & 7.1918 & 2 & 3 & 1.6271 & 8 & 16 & 7.6983 \\
\hline B13 & 4 & 4 & 4.0000 & 0.0000 & 0.00 & 156 & 241 & 69.3555 & 2 & 5 & 2.0452 & 11 & 34 & 24.6320 \\
\hline B14 & 3 & 3 & 3.1935 & 0.4016 & 0.00 & 153 & 180 & 41.3214 & 2 & 5 & 2.6700 & 10 & 28 & 12.9253 \\
\hline B15 & 1 & 1 & 1.4516 & 0.9605 & 0.00 & 152 & 167 & 20.7708 & 3 & 6 & 2.0992 & 12 & 26 & 11.3581 \\
\hline B16 & 14 & 14 & 14.0000 & 0.0000 & 0.00 & 155 & 193 & 49.9363 & 1 & 8 & 5.0855 & 5 & 47 & 33.2326 \\
\hline B17 & 13 & 13 & 13.0000 & 0.0000 & 0.00 & 153 & 179 & 33.2854 & 2 & 4 & 1.7604 & 8 & 23 & 9.0254 \\
\hline B18 & 13 & 13 & 13.0000 & 0.0000 & 0.00 & 154 & 176 & 28.2143 & 2 & 5 & 2.2442 & 8 & 26 & 12.5006 \\
\hline B19 & 13 & 13 & 13.1935 & 0.7492 & 0.00 & 153 & 205 & 61.2246 & 2 & 6 & 4.9091 & 8 & 38 & 28.1094 \\
\hline B20 & 13 & 13 & 13.0000 & 0.0000 & 0.00 & 152 & 155 & 4.7306 & 2 & 8 & 5.9932 & 10 & 35 & 24.7893 \\
\hline B21 & 9 & 9 & 9.3226 & 0.4752 & 0.00 & 155 & 167 & 12.4572 & 2 & 8 & 7.2314 & 12 & 39 & 31.2266 \\
\hline B22 & 6 & 6 & 6.1613 & 0.3739 & 0.00 & 152 & 156 & 3.6509 & 3 & 7 & 3.6945 & 12 & 29 & 15.2464 \\
\hline B23 & 6 & 6 & 6.0645 & 0.2497 & 0.00 & 152 & 155 & 4.2187 & 2 & 8 & 5.2534 & 8 & 34 & 21.9115 \\
\hline B24 & 5 & 5 & 5.4516 & 0.7229 & 0.00 & 152 & 153 & 1.5651 & 1 & 5 & 2.7048 & 4 & 24 & 11.3314 \\
\hline B25 & 4 & 4 & 4.3871 & 0.7154 & 0.00 & 152 & 153 & 1.5317 & 2 & 6 & 2.5012 & 8 & 26 & 10.3027 \\
\hline B26 & 5 & 5 & 5.0000 & 0.0000 & 0.00 & 154 & 157 & 3.2883 & 1 & 8 & 5.9864 & 4 & 36 & 24.8583 \\
\hline B27 & 3 & 3 & 3.0645 & 0.2497 & 0.00 & 152 & 156 & 3.6957 & 1 & 5 & 2.6453 & 4 & 23 & 11.2241 \\
\hline B28 & 3 & 3 & 3.3548 & 0.5507 & 0.00 & 152 & 154 & 1.6415 & 1 & 6 & 2.6506 & 4 & 25 & 11.1220 \\
\hline B29 & 3 & 3 & 3.2903 & 0.5287 & 0.00 & 152 & 153 & 1.8228 & 2 & 5 & 2.5239 & 8 & 21 & 10.6774 \\
\hline B30 & 2 & 2 & 2.4839 & 0.6768 & 0.00 & 152 & 154 & 3.7411 & 2 & 9 & 7.4944 & 10 & 36 & 29.9656 \\
\hline B31 & 7 & 7 & 7.0000 & 0.0000 & 0.00 & 154 & 158 & 3.1174 & 3 & 9 & 3.2322 & 13 & 39 & 14.2112 \\
\hline B32 & 4 & 4 & 4.0000 & 0.0000 & 0.00 & 152 & 155 & 4.6167 & 2 & 7 & 4.6467 & 8 & 31 & 19.3095 \\
\hline B33 & 4 & 4 & 4.1290 & 0.4995 & 0.00 & 152 & 154 & 2.5653 & 2 & 4 & 2.4947 & 8 & 20 & 10.4209 \\
\hline B34 & 4 & 4 & 4.1290 & 0.4995 & 0.00 & 152 & 154 & 3.5070 & 2 & 8 & 6.2436 & 8 & 36 & 25.3171 \\
\hline B35 & 4 & 4 & 4.6452 & 0.9504 & 0.00 & 152 & 153 & 1.8866 & 1 & 5 & 3.1398 & 4 & 22 & 13.0124 \\
\hline B36 & 13 & 13 & 13.0000 & 0.0000 & 0.00 & 154 & 157 & 3.0980 & 2 & 7 & 4.4587 & 8 & 32 & 19.6268 \\
\hline B37 & 10 & 10 & 10.3226 & 0.7478 & 0.00 & 153 & 156 & 2.3582 & 2 & 6 & 3.8032 & 8 & 28 & 15.7390 \\
\hline B38 & 8 & 8 & 8.0645 & 0.2497 & 0.00 & 152 & 154 & 3.6121 & 2 & 11 & 9.1926 & 9 & 47 & 36.9595 \\
\hline B39 & 5 & 5 & 5.1613 & 0.8980 & 0.00 & 152 & 153 & 1.7858 & 3 & 5 & 1.9126 & 12 & 22 & 7.5316 \\
\hline B40 & 5 & 5 & 5.1290 & 0.4995 & 0.00 & 152 & 153 & 1.5568 & 2 & 6 & 3.7757 & 8 & 26 & 15.1195 \\
\hline B41 & 8 & 8 & 8.0000 & 0.0000 & 0.00 & 154 & 158 & 4.4959 & 3 & 8 & 4.4325 & 12 & 37 & 19.3518 \\
\hline B42 & 8 & 8 & 8.0000 & 0.0000 & 0.00 & 152 & 154 & 1.8416 & 2 & 4 & 2.9146 & 8 & 19 & 11.7187 \\
\hline B43 & 8 & 8 & 8.0000 & 0.0000 & 0.00 & 152 & 153 & 1.9527 & 2 & 4 & 2.2500 & 8 & 20 & 9.6155 \\
\hline B44 & 5 & 5 & 6.0645 & 1.4591 & 0.00 & 152 & 153 & 1.9757 & 2 & 5 & 2.3859 & 8 & 23 & 9.7173 \\
\hline B45 & 5 & 5 & 6.1935 & 1.5366 & 0.00 & 152 & 154 & 3.6550 & 3 & 7 & 4.0113 & 12 & 29 & 16.0929 \\
\hline B46 & 8 & 8 & 8.0323 & 0.1796 & 0.00 & 155 & 158 & 3.2614 & 3 & 8 & 4.9381 & 13 & 35 & 20.3281 \\
\hline B47 & 5 & 5 & 5.0000 & 0.0000 & 0.00 & 152 & 155 & 1.8769 & 2 & 5 & 1.8590 & 8 & 22 & 7.6797 \\
\hline B48 & 5 & 5 & 5.0645 & 0.3592 & 0.00 & 152 & 154 & 2.0925 & 2 & 5 & 2.5012 & 8 & 22 & 10.5511 \\
\hline B49 & 5 & 5 & 5.3226 & 1.1658 & 0.00 & 152 & 155 & 3.6409 & 2 & 6 & 3.4125 & 8 & 26 & 14.2755 \\
\hline B50 & 5 & 5 & 5.3226 & 0.7478 & 0.00 & 152 & 157 & 3.3205 & 3 & 6 & 4.1309 & 12 & 28 & 16.7865 \\
\hline
\end{tabular}


Table 26: Continued.

\begin{tabular}{|c|c|c|c|c|c|c|c|c|c|c|c|c|c|c|}
\hline \multirow{2}{*}{ ID } & \multirow{2}{*}{ OPT } & \multicolumn{4}{|c|}{ Optimum } & \multicolumn{3}{|c|}{ Execution time } & \multicolumn{3}{|c|}{ Cycle } & \multicolumn{3}{|c|}{ Time cycle } \\
\hline & & $\mathrm{MBO}$ & AVG & SD & RPD\% & BET & AVG & ST & $\mathrm{BC}$ & AVG & SD & BTC & AVG & SD \\
\hline B51 & 27 & 27 & 28.0323 & 0.7521 & 0.00 & 158 & 168 & 4.9579 & 5 & 18 & 10.2663 & 21 & 82 & 45.4441 \\
\hline B52 & 18 & 18 & 18.9032 & 1.1062 & 0.00 & 155 & 163 & 4.9174 & 3 & 20 & 9.9102 & 12 & 87 & 41.3697 \\
\hline B53 & 11 & 11 & 11.0000 & 0.0000 & 0.00 & 155 & 159 & 3.0438 & 3 & 11 & 5.6777 & 13 & 48 & 23.7862 \\
\hline B54 & 11 & 11 & 11.0000 & 0.0000 & 0.00 & 152 & 158 & 3.8968 & 3 & 8 & 2.6384 & 12 & 37 & 11.8709 \\
\hline B55 & 7 & 7 & 7.0000 & 0.0000 & 0.00 & 155 & 164 & 4.9862 & 4 & 11 & 5.2009 & 16 & 51 & 23.2484 \\
\hline B56 & 6 & 6 & 6.0000 & 0.0000 & 0.00 & 154 & 160 & 5.1881 & 3 & 10 & 3.0761 & 14 & 42 & 12.8828 \\
\hline B57 & 6 & 6 & 6.0000 & 0.0000 & 0.00 & 153 & 159 & 7.0420 & 4 & 15 & 8.2207 & 16 & 64 & 35.1398 \\
\hline B58 & 6 & 6 & 6.0000 & 0.0000 & 0.00 & 152 & 159 & 8.3060 & 6 & 10 & 3.8362 & 25 & 46 & 17.9194 \\
\hline B59 & 9 & 9 & 9.0645 & 0.2497 & 0.00 & 154 & 158 & 4.4036 & 3 & 13 & 6.4481 & 13 & 57 & 25.8823 \\
\hline B 60 & 4 & 4 & 4.6452 & 1.7426 & 0.00 & 154 & 157 & 3.1356 & 3 & 11 & 6.5308 & 12 & 47 & 26.7605 \\
\hline B61 & 4 & 4 & 4.0645 & 0.2497 & 0.00 & 153 & 155 & 2.7337 & 4 & 10 & 4.5938 & 18 & 43 & 18.8219 \\
\hline B62 & 4 & 4 & 4.0000 & 0.0000 & 0.00 & 152 & 154 & 2.3592 & 3 & 10 & 4.4376 & 12 & 42 & 18.0429 \\
\hline B63 & 27 & 27 & 27.2258 & 0.4973 & 0.00 & 154 & 159 & 4.5781 & 3 & 16 & 8.9346 & 14 & 71 & 38.5607 \\
\hline B64 & 18 & 18 & 18.1613 & 0.3739 & 0.00 & 154 & 157 & 2.6036 & 2 & 21 & 10.4142 & 9 & 89 & 43.2948 \\
\hline B65 & 14 & 14 & 14.0645 & 0.2497 & 0.00 & 153 & 156 & 2.2977 & 9 & 16 & 7.2749 & 37 & 68 & 30.2108 \\
\hline B66 & 13 & 13 & 13.0000 & 0.0000 & 0.00 & 153 & 155 & 4.6939 & 4 & 9 & 4.0798 & 16 & 39 & 16.7346 \\
\hline B67 & 11 & 11 & 11.0323 & 0.1796 & 0.00 & 154 & 158 & 3.7491 & 4 & 13 & 6.3256 & 20 & 57 & 27.2279 \\
\hline B68 & 8 & 8 & 8.2581 & 1.2641 & 0.00 & 154 & 156 & 2.0780 & 4 & 14 & 8.1412 & 17 & 61 & 33.2352 \\
\hline B69 & 8 & 8 & 8.5161 & 0.5699 & 0.00 & 153 & 155 & 1.9806 & 3 & 11 & 6.3845 & 12 & 48 & 25.9051 \\
\hline B70 & 6 & 6 & 6.4839 & 0.8112 & 0.00 & 152 & 155 & 4.0201 & 6 & 14 & 7.0459 & 26 & 60 & 28.5073 \\
\hline B71 & 6 & 6 & 6.0968 & 0.3962 & 0.00 & 154 & 157 & 2.9403 & 4 & 12 & 6.6669 & 17 & 51 & 26.8024 \\
\hline B72 & 4 & 4 & 4.2258 & 0.4973 & 0.00 & 153 & 156 & 2.5425 & 4 & 12 & 4.9425 & 17 & 50 & 19.7463 \\
\hline B73 & 4 & 4 & 4.6129 & 0.8032 & 0.00 & 153 & 156 & 4.3359 & 4 & 12 & 7.1703 & 16 & 52 & 29.2444 \\
\hline B74 & 3 & 3 & 3.5806 & 0.7648 & 0.00 & 152 & 154 & 2.1597 & 3 & 12 & 6.5769 & 12 & 51 & 26.8259 \\
\hline B75 & 11 & 11 & 11.7097 & 1.0064 & 0.00 & 155 & 158 & 2.7669 & 4 & 16 & 8.1914 & 16 & 67 & 34.7627 \\
\hline B76 & 5 & 5 & 5.2581 & 0.6816 & 0.00 & 154 & 156 & 2.1938 & 4 & 15 & 8.8187 & 16 & 64 & 36.6178 \\
\hline B77 & 5 & 5 & 5.3226 & 0.7478 & 0.00 & 152 & 155 & 3.8935 & 3 & 12 & 5.2079 & 12 & 51 & 21.4117 \\
\hline B78 & 4 & 4 & 4.5806 & 1.4323 & 0.00 & 152 & 155 & 2.1292 & 5 & 16 & 7.5307 & 21 & 67 & 29.9967 \\
\hline B79 & 14 & 14 & 14.0000 & 0.0000 & 0.00 & 155 & 159 & 3.9738 & 4 & 10 & 4.1530 & 17 & 43 & 16.7283 \\
\hline B80 & 11 & 11 & 11.0000 & 0.0000 & 0.00 & 154 & 156 & 1.8293 & 6 & 11 & 4.1832 & 24 & 45 & 17.7170 \\
\hline B81 & 11 & 11 & 11.4516 & 0.9605 & 0.00 & 152 & 155 & 3.8187 & 4 & 12 & 6.0628 & 17 & 49 & 24.8217 \\
\hline B82 & 10 & 10 & 10.5484 & 0.8500 & 0.00 & 153 & 155 & 2.7044 & 3 & 11 & 6.4430 & 12 & 47 & 26.5292 \\
\hline B83 & 12 & 12 & 12.0000 & 0.0000 & 0.00 & 155 & 158 & 2.8105 & 6 & 10 & 3.6928 & 24 & 44 & 15.9765 \\
\hline B84 & 12 & 12 & 12.0000 & 0.0000 & 0.00 & 153 & 157 & 5.0225 & 2 & 10 & 4.6216 & 9 & 44 & 18.9497 \\
\hline B85 & 8 & 8 & 9.4839 & 1.6304 & 0.00 & 152 & 155 & 1.9587 & 3 & 13 & 8.1050 & 12 & 57 & 33.4782 \\
\hline B86 & 8 & 8 & 8.1290 & 0.5623 & 0.00 & 152 & 154 & 1.6611 & 3 & 11 & 4.6358 & 13 & 45 & 18.8382 \\
\hline B87 & 10 & 10 & 10.4194 & 0.8860 & 0.00 & 154 & 158 & 2.7913 & 4 & 18 & 9.6760 & 17 & 76 & 41.0048 \\
\hline B88 & 8 & 8 & 8.0000 & 0.0000 & 0.00 & 154 & 156 & 3.9358 & 5 & 10 & 3.5021 & 21 & 44 & 14.3271 \\
\hline B89 & 8 & 8 & 8.0323 & 0.1796 & 0.00 & 152 & 155 & 2.0294 & 4 & 11 & 5.5903 & 17 & 47 & 22.9967 \\
\hline \multirow[t]{2}{*}{ B90 } & 5 & 5 & 5.3871 & 1.0223 & 0.00 & 152 & 154 & 1.8334 & 5 & 11 & 5.2521 & 20 & 46 & 21.4332 \\
\hline & & AVG & 8.1007 & 0.4296 & 0.00 & 153 & 161 & 11.5292 & 2 & 9 & 4.9112 & 12 & 40 & 21.5416 \\
\hline
\end{tabular}


TABLE 27: MBO using parallel merge sort algorithm with 32 threads.

\begin{tabular}{|c|c|c|c|c|c|c|c|c|c|c|c|c|c|c|}
\hline \multirow{2}{*}{ ID } & \multirow{2}{*}{ OPT } & \multicolumn{4}{|c|}{ Optimum } & \multicolumn{3}{|c|}{ Execution time } & \multicolumn{3}{|c|}{ Cycle } & \multicolumn{3}{|c|}{ Time cycle } \\
\hline & & $\mathrm{MBO}$ & AVG & SD & RPD\% & BET & AVG & ST & $\mathrm{BC}$ & AVG & SD & BTC & AVG & SD \\
\hline B1 & 11 & 11 & 11.0000 & 0.0000 & 0.00 & 156 & 202 & 73.4610 & 3 & 7 & 2.8048 & 13 & 41 & 25.2275 \\
\hline B2 & 11 & 11 & 11.0000 & 0.0000 & 0.00 & 153 & 159 & 8.6862 & 3 & 4 & 1.4473 & 12 & 22 & 9.7667 \\
\hline B3 & 11 & 11 & 11.0000 & 0.0000 & 0.00 & 152 & 155 & 3.1575 & 1 & 4 & 1.6481 & 4 & 17 & 7.1419 \\
\hline B4 & 11 & 11 & 11.0000 & 0.0000 & 0.00 & 153 & 159 & 12.7325 & 3 & 5 & 2.2756 & 12 & 23 & 10.9487 \\
\hline B5 & 11 & 11 & 11.0000 & 0.0000 & 0.00 & 153 & 159 & 7.8248 & 2 & 5 & 2.0583 & 8 & 24 & 8.4883 \\
\hline B6 & 7 & 7 & 7.0000 & 0.0000 & 0.00 & 154 & 168 & 11.3770 & 2 & 10 & 6.2576 & 10 & 44 & 28.5622 \\
\hline B7 & 6 & 6 & 6.0645 & 0.2497 & 0.00 & 153 & 162 & 10.6669 & 3 & 6 & 2.3623 & 13 & 29 & 12.9453 \\
\hline B8 & 4 & 4 & 4.1935 & 0.6011 & 0.00 & 152 & 157 & 4.6720 & 2 & 12 & 9.9450 & 8 & 51 & 41.2599 \\
\hline B9 & 3 & 3 & 3.0968 & 0.3005 & 0.00 & 153 & 157 & 5.1856 & 1 & 5 & 2.5098 & 4 & 25 & 10.4043 \\
\hline $\mathrm{B} 10$ & 3 & 3 & 3.0000 & 0.0000 & 0.00 & 152 & 159 & 8.1494 & 1 & 3 & 1.7001 & 4 & 16 & 7.8501 \\
\hline B11 & 4 & 4 & 4.2581 & 0.4448 & 0.00 & 153 & 164 & 11.3640 & 3 & 12 & 7.4554 & 14 & 52 & 32.6614 \\
\hline B12 & 4 & 4 & 4.0000 & 0.0000 & 0.00 & 152 & 157 & 5.4880 & 1 & 4 & 1.8026 & 4 & 17 & 7.6960 \\
\hline B13 & 4 & 4 & 4.0645 & 0.3592 & 0.00 & 152 & 159 & 9.9945 & 2 & 5 & 2.4679 & 9 & 22 & 10.6621 \\
\hline B14 & 3 & 3 & 3.2581 & 0.4448 & 0.00 & 152 & 157 & 7.2963 & 2 & 5 & 2.6632 & 8 & 23 & 11.1257 \\
\hline B15 & 1 & 1 & 1.5161 & 0.8896 & 0.00 & 152 & 162 & 14.1797 & 2 & 6 & 2.7731 & 8 & 25 & 12.0180 \\
\hline B16 & 14 & 14 & 14.0000 & 0.0000 & 0.00 & 155 & 178 & 28.1446 & 3 & 6 & 2.8459 & 12 & 32 & 12.6052 \\
\hline B17 & 13 & 13 & 13.0000 & 0.0000 & 0.00 & 152 & 163 & 9.4999 & 1 & 4 & 1.6669 & 4 & 19 & 7.8038 \\
\hline B18 & 13 & 13 & 13.0000 & 0.0000 & 0.00 & 153 & 176 & 50.4821 & 1 & 5 & 2.3431 & 5 & 22 & 12.0773 \\
\hline B19 & 13 & 13 & 13.1935 & 0.7492 & 0.00 & 153 & 167 & 23.5020 & 2 & 7 & 4.6575 & 12 & 34 & 25.0622 \\
\hline B20 & 13 & 13 & 13.0000 & 0.0000 & 0.00 & 152 & 196 & 65.9215 & 2 & 7 & 4.9246 & 9 & 39 & 27.7927 \\
\hline B21 & 9 & 9 & 9.3548 & 0.4864 & 0.00 & 154 & 197 & 89.8626 & 2 & 11 & 8.3362 & 8 & 56 & 40.0088 \\
\hline B22 & 6 & 6 & 6.1613 & 0.5829 & 0.00 & 155 & 213 & 63.0063 & 2 & 5 & 2.0161 & 8 & 32 & 18.9462 \\
\hline B23 & 6 & 6 & 6.1935 & 0.4016 & 0.00 & 155 & 241 & 100.2556 & 3 & 7 & 5.8505 & 12 & 48 & 39.5844 \\
\hline B24 & 5 & 5 & 5.2903 & 0.5884 & 0.00 & 152 & 164 & 31.9873 & 2 & 6 & 3.1288 & 8 & 28 & 12.6167 \\
\hline B25 & 4 & 4 & 4.1613 & 0.3739 & 0.00 & 152 & 155 & 2.4839 & 2 & 6 & 4.9858 & 8 & 26 & 20.2954 \\
\hline B26 & 5 & 5 & 5.0645 & 0.2497 & 0.00 & 155 & 162 & 5.6949 & 2 & 7 & 5.6531 & 8 & 34 & 24.0685 \\
\hline B27 & 3 & 3 & 3.0968 & 0.3005 & 0.00 & 154 & 158 & 2.8860 & 2 & 6 & 3.6580 & 9 & 28 & 15.0662 \\
\hline B28 & 3 & 3 & 3.1613 & 0.4544 & 0.00 & 152 & 156 & 3.2326 & 2 & 7 & 3.2322 & 8 & 30 & 13.6381 \\
\hline B29 & 3 & 3 & 3.0968 & 0.3005 & 0.00 & 152 & 155 & 3.9811 & 2 & 5 & 2.2785 & 8 & 23 & 9.0958 \\
\hline B30 & 2 & 2 & 2.5161 & 0.6256 & 0.00 & 152 & 156 & 4.9036 & 2 & 7 & 5.6843 & 8 & 30 & 23.2274 \\
\hline B31 & 7 & 7 & 7.0000 & 0.0000 & 0.00 & 155 & 164 & 5.3710 & 1 & 7 & 4.3247 & 4 & 33 & 19.4109 \\
\hline B32 & 4 & 4 & 4.1935 & 0.7492 & 0.00 & 152 & 157 & 3.1191 & 2 & 6 & 3.7035 & 8 & 28 & 15.7836 \\
\hline B33 & 4 & 4 & 4.0000 & 0.0000 & 0.00 & 153 & 156 & 2.9574 & 3 & 5 & 2.7712 & 12 & 23 & 11.8504 \\
\hline B34 & 4 & 4 & 4.0645 & 0.3592 & 0.00 & 152 & 156 & 4.1986 & 2 & 7 & 7.1646 & 8 & 32 & 31.8354 \\
\hline B35 & 4 & 4 & 4.3226 & 0.7478 & 0.00 & 152 & 155 & 3.3758 & 2 & 5 & 3.0862 & 8 & 22 & 12.7461 \\
\hline B36 & 13 & 13 & 13.0000 & 0.0000 & 0.00 & 155 & 162 & 5.9413 & 2 & 7 & 3.9284 & 9 & 34 & 16.4346 \\
\hline B37 & 10 & 10 & 10.3226 & 0.7478 & 0.00 & 154 & 157 & 3.3153 & 2 & 7 & 5.8622 & 8 & 29 & 23.8995 \\
\hline B38 & 8 & 8 & 8.2581 & 1.2641 & 0.00 & 152 & 156 & 4.6789 & 2 & 10 & 8.4328 & 8 & 42 & 34.3955 \\
\hline B39 & 5 & 5 & 5.0000 & 0.0000 & 0.00 & 152 & 159 & 6.1503 & 2 & 5 & 2.9153 & 8 & 25 & 12.1929 \\
\hline B40 & 5 & 5 & 5.1290 & 0.4995 & 0.00 & 155 & 168 & 23.4268 & 3 & 5 & 1.5513 & 13 & 23 & 7.9119 \\
\hline B41 & 8 & 8 & 8.1613 & 0.8980 & 0.00 & 154 & 161 & 4.2011 & 4 & 9 & 6.6078 & 16 & 42 & 27.6753 \\
\hline B42 & 8 & 8 & 8.0000 & 0.0000 & 0.00 & 153 & 157 & 6.3106 & 2 & 4 & 1.7828 & 8 & 18 & 7.7493 \\
\hline B43 & 8 & 8 & 8.0000 & 0.0000 & 0.00 & 152 & 160 & 7.4285 & 2 & 5 & 2.6019 & 8 & 23 & 11.7932 \\
\hline B44 & 5 & 5 & 5.8710 & 1.3842 & 0.00 & 154 & 161 & 7.9767 & 2 & 5 & 2.0687 & 12 & 22 & 9.0735 \\
\hline B45 & 5 & 5 & 5.8710 & 1.3842 & 0.00 & 153 & 156 & 5.2525 & 2 & 5 & 3.0603 & 8 & 25 & 12.6925 \\
\hline B46 & 8 & 8 & 8.0323 & 0.1796 & 0.00 & 154 & 165 & 8.5232 & 1 & 9 & 7.2947 & 4 & 40 & 32.4977 \\
\hline B47 & 5 & 5 & 5.0000 & 0.0000 & 0.00 & 153 & 163 & 7.4939 & 2 & 6 & 3.3732 & 8 & 27 & 13.6618 \\
\hline B48 & 5 & 5 & 5.0645 & 0.3592 & 0.00 & 155 & 190 & 88.3159 & 3 & 5 & 2.3550 & 12 & 26 & 10.0136 \\
\hline B49 & 5 & 5 & 5.1290 & 0.4995 & 0.00 & 153 & 167 & 23.7535 & 2 & 6 & 2.2562 & 8 & 25 & 9.6192 \\
\hline B50 & 5 & 5 & 5.1935 & 0.6011 & 0.00 & 153 & 206 & 111.6447 & 1 & 5 & 2.3619 & 4 & 30 & 22.2696 \\
\hline
\end{tabular}


TABle 27: Continued.

\begin{tabular}{|c|c|c|c|c|c|c|c|c|c|c|c|c|c|c|}
\hline \multirow{2}{*}{ ID } & \multirow{2}{*}{ OPT } & \multicolumn{4}{|c|}{ Optimum } & \multicolumn{3}{|c|}{ Execution time } & \multicolumn{3}{|c|}{ Cycle } & \multicolumn{3}{|c|}{ Time cycle } \\
\hline & & $\mathrm{MBO}$ & AVG & SD & $\mathrm{RPD} \%$ & $\mathrm{BET}$ & AVG & ST & $\mathrm{BC}$ & AVG & $\mathrm{SD}$ & BTC & AVG & SD \\
\hline B51 & 27 & 27 & 28.1613 & 0.7347 & 0.00 & 160 & 233 & 106.5829 & 4 & 19 & 9.9746 & 16 & 113 & 64.2842 \\
\hline B52 & 18 & 18 & 18.8387 & 0.7347 & 0.00 & 154 & 164 & 6.9436 & 6 & 21 & 9.2897 & 27 & 91 & 41.3948 \\
\hline B53 & 11 & 11 & 11.0000 & 0.0000 & 0.00 & 155 & 164 & 13.5560 & 5 & 11 & 3.1833 & 22 & 47 & 13.7809 \\
\hline B54 & 11 & 11 & 11.0000 & 0.0000 & 0.00 & 156 & 163 & 4.5475 & 4 & 8 & 2.4274 & 17 & 37 & 11.2196 \\
\hline B55 & 7 & 7 & 7.0000 & 0.0000 & 0.00 & 159 & 170 & 8.7338 & 5 & 10 & 3.2038 & 26 & 47 & 13.2918 \\
\hline B56 & 6 & 6 & 6.0000 & 0.0000 & 0.00 & 154 & 166 & 10.0578 & 6 & 10 & 3.3742 & 27 & 47 & 16.6980 \\
\hline B57 & 6 & 6 & 6.0000 & 0.0000 & 0.00 & 155 & 169 & 18.2314 & 6 & 11 & 5.5162 & 25 & 52 & 24.6267 \\
\hline B58 & 6 & 6 & 6.0000 & 0.0000 & 0.00 & 154 & 162 & 4.3901 & 3 & 10 & 5.1287 & 12 & 44 & 22.2686 \\
\hline B59 & 9 & 9 & 9.1613 & 0.5829 & 0.00 & 161 & 176 & 14.3747 & 5 & 14 & 7.2798 & 23 & 70 & 36.4352 \\
\hline $\mathrm{B} 60$ & 4 & 4 & 4.2903 & 1.1312 & 0.00 & 157 & 173 & 14.4923 & 6 & 14 & 6.1914 & 25 & 64 & 28.9730 \\
\hline B61 & 4 & 4 & 4.1613 & 0.5829 & 0.00 & 155 & 163 & 6.7429 & 4 & 9 & 4.0040 & 16 & 42 & 16.5396 \\
\hline B62 & 4 & 4 & 4.0323 & 0.1796 & 0.00 & 154 & 160 & 3.5345 & 4 & 10 & 4.6504 & 16 & 43 & 19.3696 \\
\hline B63 & 27 & 27 & 27.2258 & 0.4250 & 0.00 & 159 & 172 & 5.1895 & 3 & 20 & 11.1084 & 13 & 91 & 50.3296 \\
\hline B64 & 18 & 18 & 18.1935 & 0.4016 & 0.00 & 157 & 173 & 22.8970 & 6 & 21 & 9.5444 & 25 & 102 & 51.5291 \\
\hline B65 & 14 & 14 & 14.0645 & 0.2497 & 0.00 & 156 & 229 & 88.8105 & 6 & 16 & 6.8318 & 29 & 96 & 56.9926 \\
\hline B66 & 13 & 13 & 13.0000 & 0.0000 & 0.00 & 155 & 198 & 64.6472 & 4 & 10 & 4.2088 & 20 & 55 & 27.5365 \\
\hline B67 & 11 & 11 & 11.0968 & 0.3005 & 0.00 & 166 & 269 & 216.8374 & 3 & 15 & 9.5772 & 14 & 118 & 195.6026 \\
\hline B68 & 8 & 8 & 8.2258 & 0.9205 & 0.00 & 154 & 170 & 13.6269 & 4 & 11 & 6.2202 & 17 & 53 & 26.0361 \\
\hline B69 & 8 & 8 & 8.5161 & 0.7244 & 0.00 & 155 & 168 & 15.0527 & 6 & 13 & 6.1990 & 26 & 59 & 36.8672 \\
\hline B70 & 6 & 6 & 6.5484 & 0.8884 & 0.00 & 153 & 156 & 5.1117 & 3 & 14 & 8.3743 & 14 & 62 & 34.9847 \\
\hline B71 & 6 & 6 & 6.1290 & 0.4995 & 0.00 & 154 & 160 & 5.1506 & 4 & 11 & 4.5231 & 16 & 49 & 19.3207 \\
\hline B72 & 4 & 4 & 4.3548 & 0.5507 & 0.00 & 152 & 157 & 3.9059 & 6 & 16 & 8.5049 & 25 & 67 & 36.2737 \\
\hline B73 & 4 & 4 & 4.5161 & 0.6768 & 0.00 & 152 & 166 & 25.8682 & 4 & 13 & 9.0983 & 17 & 57 & 39.4182 \\
\hline B74 & 3 & 3 & 3.5484 & 0.7676 & 0.00 & 154 & 169 & 18.5330 & 3 & 13 & 7.3941 & 13 & 57 & 32.0482 \\
\hline B75 & 11 & 11 & 11.6452 & 0.9504 & 0.00 & 156 & 166 & 10.2917 & 6 & 14 & 9.4745 & 26 & 64 & 42.2402 \\
\hline B76 & 5 & 5 & 5.1290 & 0.4995 & 0.00 & 153 & 202 & 49.9134 & 6 & 13 & 6.4288 & 25 & 73 & 35.4865 \\
\hline B77 & 5 & 5 & 5.3871 & 0.8032 & 0.00 & 152 & 161 & 11.1016 & 4 & 13 & 7.0557 & 17 & 56 & 28.8527 \\
\hline B78 & 4 & 4 & 4.7742 & 1.3344 & 0.00 & 153 & 159 & 7.9270 & 4 & 13 & 6.6488 & 17 & 55 & 29.2830 \\
\hline B79 & 14 & 14 & 14.0000 & 0.0000 & 0.00 & 153 & 166 & 11.9017 & 2 & 9 & 3.9646 & 8 & 41 & 16.3156 \\
\hline B80 & 11 & 11 & 11.0000 & 0.0000 & 0.00 & 154 & 162 & 9.6500 & 6 & 11 & 5.3587 & 24 & 50 & 23.5722 \\
\hline B81 & 11 & 11 & 11.3548 & 0.8386 & 0.00 & 153 & 158 & 6.6857 & 5 & 13 & 7.0744 & 20 & 56 & 29.7124 \\
\hline B82 & 10 & 10 & 10.4516 & 0.7676 & 0.00 & 152 & 160 & 10.6532 & 5 & 13 & 5.9386 & 20 & 55 & 27.4567 \\
\hline B83 & 12 & 12 & 12.0968 & 0.5388 & 0.00 & 153 & 170 & 19.9290 & 5 & 11 & 3.8451 & 24 & 51 & 17.9308 \\
\hline B84 & 12 & 12 & 12.0000 & 0.0000 & 0.00 & 154 & 161 & 6.7725 & 4 & 13 & 7.3941 & 16 & 55 & 31.6225 \\
\hline B85 & 8 & 8 & 8.8387 & 1.2409 & 0.00 & 154 & 164 & 14.7388 & 5 & 14 & 7.2984 & 22 & 62 & 34.7666 \\
\hline B86 & 8 & 8 & 8.0968 & 0.5388 & 0.00 & 153 & 162 & 10.3043 & 4 & 10 & 4.3183 & 16 & 44 & 18.7357 \\
\hline B87 & 10 & 10 & 10.1290 & 0.4995 & 0.00 & 154 & 166 & 10.6363 & 5 & 18 & 8.1722 & 21 & 80 & 36.7600 \\
\hline B88 & 8 & 8 & 8.0000 & 0.0000 & 0.00 & 154 & 160 & 6.2300 & 6 & 11 & 4.4717 & 24 & 50 & 18.2966 \\
\hline B89 & 8 & 8 & 8.0000 & 0.0000 & 0.00 & 154 & 181 & 63.1321 & 4 & 10 & 3.9393 & 16 & 50 & 22.3958 \\
\hline \multirow[t]{2}{*}{ B90 } & 5 & 5 & 5.4839 & 1.1216 & 0.00 & 154 & 158 & 5.3563 & 3 & 10 & 4.3045 & 12 & 41 & 18.0081 \\
\hline & & AVG & 8.0756 & 0.4173 & 0.00 & 154 & 170 & 21.8506 & 3 & 9 & 4.8937 & 14 & 44 & 24.7712 \\
\hline
\end{tabular}


TABLE 28: MBO using sequential quick sort algorithm.

\begin{tabular}{|c|c|c|c|c|c|c|c|c|c|c|c|c|c|c|}
\hline \multirow{2}{*}{ ID } & \multirow{2}{*}{ OPT } & \multicolumn{4}{|c|}{ Optimum } & \multicolumn{3}{|c|}{ Execution time } & \multicolumn{3}{|c|}{ Cycle } & \multicolumn{3}{|c|}{ Time cycle } \\
\hline & & $\mathrm{MBO}$ & AVG & SD & RPD\% & BET & AVG & ST & $\mathrm{BC}$ & AVG & SD & BTC & AVG & SD \\
\hline B1 & 11 & 11 & 11.0000 & 0.0000 & 0.00 & 156 & 190 & 58.2650 & 2 & 7 & 3.8624 & 11 & 43 & 33.3476 \\
\hline B2 & 11 & 11 & 11.0000 & 0.0000 & 0.00 & 153 & 161 & 11.6192 & 2 & 4 & 1.4074 & 8 & 19 & 6.0245 \\
\hline B3 & 11 & 11 & 11.0000 & 0.0000 & 0.00 & 152 & 155 & 2.5085 & 2 & 4 & 1.5176 & 8 & 17 & 6.5214 \\
\hline B4 & 11 & 11 & 11.0000 & 0.0000 & 0.00 & 152 & 182 & 101.4110 & 2 & 4 & 1.8246 & 8 & 22 & 9.4797 \\
\hline B5 & 11 & 11 & 11.0000 & 0.0000 & 0.00 & 152 & 156 & 6.3229 & 2 & 5 & 3.4426 & 9 & 22 & 13.9587 \\
\hline B6 & 7 & 7 & 7.0000 & 0.0000 & 0.00 & 154 & 161 & 13.5107 & 3 & 8 & 4.1693 & 12 & 37 & 17.3353 \\
\hline B7 & 6 & 6 & 6.0323 & 0.1796 & 0.00 & 153 & 168 & 13.8935 & 3 & 6 & 2.9068 & 12 & 31 & 13.1726 \\
\hline B8 & 4 & 4 & 4.0645 & 0.3592 & 0.00 & 152 & 182 & 34.6447 & 2 & 10 & 7.8375 & 8 & 49 & 35.8762 \\
\hline B9 & 3 & 3 & 3.1290 & 0.3408 & 0.00 & 152 & 156 & 9.6349 & 2 & 6 & 5.9664 & 8 & 29 & 33.1260 \\
\hline $\mathrm{B} 10$ & 3 & 3 & 3.0000 & 0.0000 & 0.00 & 152 & 154 & 2.1771 & 2 & 4 & 1.1968 & 8 & 16 & 4.7947 \\
\hline B11 & 4 & 4 & 4.1290 & 0.3408 & 0.00 & 154 & 157 & 3.1084 & 1 & 11 & 8.3456 & 4 & 46 & 34.7346 \\
\hline B12 & 4 & 4 & 4.0000 & 0.0000 & 0.00 & 152 & 155 & 3.0374 & 1 & 3 & 1.5794 & 5 & 15 & 6.4709 \\
\hline B13 & 4 & 4 & 4.2258 & 0.7169 & 0.00 & 152 & 153 & 1.8814 & 2 & 5 & 5.3585 & 8 & 23 & 22.5828 \\
\hline B14 & 3 & 3 & 3.3548 & 0.4864 & 0.00 & 152 & 154 & 1.6533 & 2 & 5 & 2.3971 & 8 & 23 & 9.8987 \\
\hline B15 & 1 & 1 & 1.1290 & 0.4995 & 0.00 & 152 & 153 & 1.5027 & 2 & 6 & 2.2912 & 8 & 24 & 9.3735 \\
\hline B16 & 14 & 14 & 14.0000 & 0.0000 & 0.00 & 154 & 158 & 6.5646 & 2 & 8 & 4.7026 & 8 & 34 & 19.1483 \\
\hline B17 & 13 & 13 & 13.0000 & 0.0000 & 0.00 & 153 & 155 & 2.0283 & 2 & 5 & 1.8860 & 9 & 21 & 7.4763 \\
\hline B18 & 13 & 13 & 13.0000 & 0.0000 & 0.00 & 152 & 157 & 7.3231 & 1 & 6 & 2.4995 & 4 & 25 & 10.3130 \\
\hline B19 & 13 & 13 & 13.0968 & 0.5388 & 0.00 & 152 & 159 & 6.4381 & 2 & 7 & 6.0690 & 8 & 33 & 24.6737 \\
\hline B20 & 13 & 13 & 13.0000 & 0.0000 & 0.00 & 153 & 159 & 7.5257 & 2 & 7 & 4.6504 & 8 & 31 & 19.5123 \\
\hline B21 & 9 & 9 & 9.2903 & 0.4614 & 0.00 & 154 & 158 & 4.8569 & 2 & 9 & 7.8941 & 8 & 41 & 32.4426 \\
\hline B22 & 6 & 6 & 6.1613 & 0.3739 & 0.00 & 152 & 160 & 7.6086 & 3 & 6 & 2.7611 & 12 & 26 & 12.0577 \\
\hline B23 & 6 & 6 & 6.1613 & 0.3739 & 0.00 & 152 & 157 & 5.0575 & 4 & 10 & 7.1245 & 16 & 43 & 29.3833 \\
\hline B24 & 5 & 5 & 5.2903 & 0.5287 & 0.00 & 152 & 159 & 6.8078 & 2 & 7 & 4.8146 & 9 & 30 & 19.7313 \\
\hline B25 & 4 & 4 & 4.1613 & 0.3739 & 0.00 & 152 & 155 & 4.9514 & 2 & 6 & 2.5430 & 8 & 24 & 10.4495 \\
\hline B26 & 5 & 5 & 5.0000 & 0.0000 & 0.00 & 154 & 163 & 8.4537 & 2 & 8 & 4.4837 & 8 & 36 & 18.4994 \\
\hline B27 & 3 & 3 & 3.0323 & 0.1796 & 0.00 & 153 & 158 & 6.3527 & 1 & 7 & 5.5072 & 5 & 29 & 22.6066 \\
\hline B28 & 3 & 3 & 3.2581 & 0.4448 & 0.00 & 152 & 162 & 9.1764 & 2 & 6 & 4.4041 & 8 & 26 & 20.0669 \\
\hline B29 & 3 & 3 & 3.1613 & 0.3739 & 0.00 & 152 & 163 & 8.9060 & 1 & 5 & 2.9534 & 4 & 23 & 12.2417 \\
\hline B30 & 2 & 2 & 2.6129 & 0.8032 & 0.00 & 152 & 162 & 11.2350 & 2 & 7 & 6.3716 & 8 & 30 & 26.7117 \\
\hline B31 & 7 & 7 & 7.0000 & 0.0000 & 0.00 & 155 & 172 & 13.0047 & 3 & 8 & 5.2176 & 14 & 40 & 23.0526 \\
\hline B32 & 4 & 4 & 4.0968 & 0.5388 & 0.00 & 154 & 163 & 16.2313 & 2 & 8 & 5.0189 & 8 & 36 & 22.6064 \\
\hline B33 & 4 & 4 & 4.0645 & 0.3592 & 0.00 & 153 & 161 & 8.5798 & 2 & 5 & 2.5016 & 8 & 22 & 10.6692 \\
\hline B34 & 4 & 4 & 4.1290 & 0.4995 & 0.00 & 152 & 158 & 9.0177 & 2 & 6 & 5.0302 & 8 & 27 & 20.6482 \\
\hline B35 & 4 & 4 & 4.6452 & 0.9504 & 0.00 & 152 & 153 & 1.5098 & 1 & 5 & 4.4678 & 4 & 23 & 18.0923 \\
\hline B36 & 13 & 13 & 13.0000 & 0.0000 & 0.00 & 154 & 174 & 26.3191 & 3 & 8 & 4.2014 & 12 & 41 & 24.8909 \\
\hline B37 & 10 & 10 & 10.5161 & 0.8896 & 0.00 & 153 & 161 & 12.2450 & 2 & 7 & 3.9136 & 8 & 30 & 15.9159 \\
\hline B38 & 8 & 8 & 8.0968 & 0.3962 & 0.00 & 152 & 157 & 6.5512 & 2 & 10 & 8.7204 & 8 & 43 & 35.7477 \\
\hline B39 & 5 & 5 & 5.0000 & 0.0000 & 0.00 & 152 & 161 & 13.6204 & 3 & 5 & 2.3431 & 12 & 25 & 10.6618 \\
\hline B40 & 5 & 5 & 5.0000 & 0.0000 & 0.00 & 152 & 163 & 9.7288 & 2 & 5 & 2.1438 & 8 & 21 & 9.9864 \\
\hline B41 & 8 & 8 & 8.0000 & 0.0000 & 0.00 & 155 & 165 & 12.7196 & 1 & 10 & 6.2553 & 5 & 48 & 26.9376 \\
\hline B42 & 8 & 8 & 8.0000 & 0.0000 & 0.00 & 152 & 157 & 3.5951 & 2 & 4 & 1.7804 & 8 & 19 & 7.5267 \\
\hline B43 & 8 & 8 & 8.0645 & 0.3592 & 0.00 & 152 & 155 & 2.4947 & 2 & 5 & 5.3110 & 8 & 24 & 22.0386 \\
\hline B44 & 5 & 5 & 6.0645 & 1.4591 & 0.00 & 152 & 155 & 3.3302 & 2 & 6 & 3.3601 & 8 & 25 & 13.7010 \\
\hline B45 & 5 & 5 & 6.4516 & 1.5240 & 0.00 & 152 & 154 & 1.4135 & 2 & 5 & 2.2907 & 8 & 21 & 9.2427 \\
\hline B46 & 8 & 8 & 8.1290 & 0.3408 & 0.00 & 154 & 161 & 4.7801 & 3 & 9 & 6.7587 & 14 & 42 & 29.0482 \\
\hline B47 & 5 & 5 & 5.0968 & 0.5388 & 0.00 & 153 & 156 & 2.5392 & 1 & 4 & 1.8139 & 4 & 20 & 7.8833 \\
\hline B48 & 5 & 5 & 5.1935 & 0.6011 & 0.00 & 152 & 155 & 4.2327 & 2 & 5 & 2.4021 & 8 & 23 & 9.6806 \\
\hline B49 & 5 & 5 & 5.1290 & 0.4995 & 0.00 & 152 & 155 & 3.8960 & 3 & 7 & 4.9298 & 12 & 31 & 20.3514 \\
\hline B50 & 5 & 5 & 5.1935 & 0.6011 & 0.00 & 152 & 157 & 7.6718 & 2 & 5 & 2.2728 & 8 & 24 & 9.2511 \\
\hline
\end{tabular}


TABle 28: Continued.

\begin{tabular}{|c|c|c|c|c|c|c|c|c|c|c|c|c|c|c|}
\hline \multirow{2}{*}{ ID } & \multirow{2}{*}{ OPT } & \multicolumn{4}{|c|}{ Optimum } & \multicolumn{3}{|c|}{ Execution time } & \multicolumn{3}{|c|}{ Cycle } & \multicolumn{3}{|c|}{ Time cycle } \\
\hline & & $\mathrm{MBO}$ & AVG & $\mathrm{SD}$ & RPD\% & BET & AVG & ST & $\mathrm{BC}$ & AVG & $\mathrm{SD}$ & BTC & AVG & $\mathrm{SD}$ \\
\hline B51 & 27 & 27 & 28.2258 & 0.9205 & 0.00 & 155 & 161 & 4.3769 & 4 & 19 & 9.2586 & 20 & 82 & 39.4718 \\
\hline B52 & 18 & 18 & 18.9032 & 1.1062 & 0.00 & 154 & 158 & 4.4286 & 7 & 20 & 9.4888 & 29 & 85 & 40.2330 \\
\hline B53 & 11 & 11 & 11.0000 & 0.0000 & 0.00 & 154 & 157 & 2.8269 & 4 & 10 & 4.1346 & 16 & 44 & 16.8027 \\
\hline B54 & 11 & 11 & 11.0000 & 0.0000 & 0.00 & 153 & 156 & 2.3075 & 4 & 9 & 3.2052 & 16 & 38 & 13.3121 \\
\hline B55 & 7 & 7 & 7.0000 & 0.0000 & 0.00 & 154 & 158 & 2.9370 & 4 & 10 & 5.4839 & 16 & 45 & 22.7564 \\
\hline B56 & 6 & 6 & 6.0000 & 0.0000 & 0.00 & 154 & 157 & 2.2337 & 4 & 8 & 2.6184 & 16 & 36 & 11.1519 \\
\hline B57 & 6 & 6 & 6.0000 & 0.0000 & 0.00 & 153 & 157 & 4.3503 & 6 & 10 & 4.0231 & 24 & 43 & 16.3044 \\
\hline B58 & 6 & 6 & 6.1290 & 0.3408 & 0.00 & 154 & 157 & 2.8189 & 5 & 10 & 3.8882 & 20 & 44 & 15.7987 \\
\hline B59 & 9 & 9 & 9.1935 & 0.6011 & 0.00 & 154 & 160 & 3.4817 & 3 & 13 & 8.1588 & 12 & 59 & 34.8749 \\
\hline B60 & 4 & 4 & 4.5161 & 1.3631 & 0.00 & 154 & 157 & 2.8511 & 3 & 12 & 4.7288 & 12 & 50 & 19.8736 \\
\hline B61 & 4 & 4 & 4.2258 & 0.6170 & 0.00 & 153 & 156 & 3.6237 & 3 & 11 & 4.2571 & 17 & 48 & 17.2788 \\
\hline B62 & 4 & 4 & 4.0323 & 0.1796 & 0.00 & 152 & 155 & 2.5098 & 4 & 10 & 3.6820 & 16 & 41 & 15.5667 \\
\hline B63 & 27 & 27 & 27.2581 & 0.4448 & 0.00 & 155 & 164 & 6.3053 & 4 & 19 & 10.1183 & 19 & 84 & 42.8466 \\
\hline B64 & 18 & 18 & 18.3548 & 0.5507 & 0.00 & 154 & 175 & 29.3106 & 7 & 20 & 9.2391 & 37 & 95 & 41.8859 \\
\hline B65 & 14 & 14 & 14.1935 & 0.4016 & 0.00 & 154 & 162 & 11.5242 & 5 & 14 & 6.2896 & 22 & 61 & 26.2881 \\
\hline B66 & 13 & 13 & 13.0000 & 0.0000 & 0.00 & 154 & 174 & 31.3824 & 6 & 11 & 3.7301 & 24 & 51 & 22.7029 \\
\hline B67 & 11 & 11 & 11.0323 & 0.1796 & 0.00 & 155 & 166 & 11.5943 & 6 & 12 & 5.7328 & 24 & 53 & 23.5936 \\
\hline B68 & 8 & 8 & 8.5806 & 1.7469 & 0.00 & 154 & 160 & 5.9015 & 3 & 11 & 6.2869 & 12 & 48 & 25.7106 \\
\hline B69 & 8 & 8 & 8.5161 & 0.8112 & 0.00 & 153 & 165 & 12.9041 & 4 & 10 & 5.4759 & 16 & 47 & 24.7696 \\
\hline B70 & 6 & 6 & 6.6452 & 0.8386 & 0.00 & 153 & 160 & 11.3877 & 2 & 12 & 7.7095 & 8 & 52 & 31.6072 \\
\hline B71 & 6 & 6 & 6.1290 & 0.4995 & 0.00 & 157 & 178 & 18.6546 & 2 & 11 & 5.3096 & 11 & 54 & 28.3288 \\
\hline B72 & 4 & 4 & 4.2581 & 0.7732 & 0.00 & 154 & 171 & 19.4453 & 4 & 11 & 6.3664 & 16 & 54 & 29.4289 \\
\hline B73 & 4 & 4 & 4.4194 & 0.6204 & 0.00 & 154 & 174 & 16.4459 & 5 & 10 & 4.0422 & 21 & 51 & 19.9695 \\
\hline B74 & 3 & 3 & 3.5484 & 0.6239 & 0.00 & 156 & 187 & 33.9678 & 5 & 12 & 7.8744 & 20 & 67 & 49.6365 \\
\hline B75 & 11 & 11 & 11.5806 & 1.0255 & 0.00 & 159 & 200 & 45.3931 & 4 & 17 & 9.9043 & 17 & 95 & 67.7548 \\
\hline B76 & 5 & 5 & 5.0645 & 0.3592 & 0.00 & 154 & 171 & 37.0215 & 4 & 14 & 6.9785 & 17 & 67 & 30.3144 \\
\hline B77 & 5 & 5 & 5.3871 & 0.8032 & 0.00 & 154 & 161 & 7.2560 & 4 & 14 & 7.2969 & 17 & 62 & 31.3623 \\
\hline B78 & 4 & 4 & 4.5806 & 1.1188 & 0.00 & 153 & 166 & 11.1021 & 8 & 15 & 4.9588 & 32 & 69 & 24.4737 \\
\hline B79 & 14 & 14 & 14.0000 & 0.0000 & 0.00 & 155 & 173 & 15.1100 & 5 & 9 & 2.9421 & 20 & 44 & 16.3185 \\
\hline B80 & 11 & 11 & 11.0000 & 0.0000 & 0.00 & 154 & 164 & 13.3813 & 4 & 11 & 3.7198 & 17 & 48 & 16.2591 \\
\hline B81 & 11 & 11 & 11.5484 & 1.1787 & 0.00 & 155 & 177 & 20.2888 & 2 & 13 & 8.5572 & 8 & 64 & 39.3331 \\
\hline B82 & 10 & 10 & 10.7419 & 0.7732 & 0.00 & 154 & 227 & 142.7347 & 3 & 10 & 5.1533 & 13 & 56 & 31.9425 \\
\hline B83 & 12 & 12 & 12.3548 & 1.5176 & 0.00 & 154 & 180 & 28.3520 & 3 & 11 & 6.7907 & 13 & 58 & 36.4259 \\
\hline B84 & 12 & 12 & 12.0000 & 0.0000 & 0.00 & 155 & 165 & 12.8809 & 2 & 12 & 5.0773 & 9 & 54 & 23.5388 \\
\hline B85 & 8 & 8 & 9.4516 & 1.6899 & 0.00 & 154 & 164 & 17.4202 & 3 & 13 & 6.9161 & 13 & 59 & 29.5533 \\
\hline B86 & 8 & 8 & 8.1613 & 0.5829 & 0.00 & 153 & 155 & 2.0525 & 4 & 11 & 6.3534 & 16 & 49 & 25.9607 \\
\hline B87 & 10 & 10 & 10.3871 & 0.9549 & 0.00 & 154 & 159 & 5.2984 & 5 & 16 & 9.5733 & 20 & 68 & 40.5421 \\
\hline B88 & 8 & 8 & 8.0000 & 0.0000 & 0.00 & 154 & 157 & 2.8466 & 5 & 10 & 3.0610 & 21 & 43 & 12.7689 \\
\hline B89 & 8 & 8 & 8.0323 & 0.1796 & 0.00 & 154 & 156 & 2.3349 & 4 & 10 & 5.9039 & 16 & 43 & 23.9970 \\
\hline \multirow[t]{2}{*}{ B90 } & 5 & 5 & 5.3871 & 1.0223 & 0.00 & 153 & 155 & 2.5778 & 3 & 11 & 4.7201 & 13 & 46 & 18.9533 \\
\hline & & AVG & 8.1111 & 0.4529 & 0.00 & 153 & 163 & 12.7284 & 2 & 9 & 4.9168 & 12 & 41 & 22.1071 \\
\hline
\end{tabular}


TABLE 29: MBO using parallel quick sort algorithm with 1 thread.

\begin{tabular}{|c|c|c|c|c|c|c|c|c|c|c|c|c|c|c|}
\hline \multirow{2}{*}{ ID } & \multirow{2}{*}{ OPT } & \multicolumn{4}{|c|}{ Optimum } & \multicolumn{3}{|c|}{ Execution time } & \multicolumn{3}{|c|}{ Cycle } & \multicolumn{3}{|c|}{ Time cycle } \\
\hline & & $\mathrm{MBO}$ & AVG & SD & RPD\% & BET & AVG & ST & $\mathrm{BC}$ & AVG & $\mathrm{SD}$ & BTC & AVG & $\mathrm{SD}$ \\
\hline B1 & 11 & 11 & 11.0000 & 0.0000 & 0.00 & 156 & 193 & 68.9999 & 2 & 7 & 3.1195 & 9 & 41 & 17.8353 \\
\hline B2 & 11 & 11 & 11.0000 & 0.0000 & 0.00 & 153 & 165 & 18.8674 & 2 & 4 & 1.1538 & 8 & 19 & 8.8834 \\
\hline B3 & 11 & 11 & 11.0000 & 0.0000 & 0.00 & 153 & 161 & 19.3106 & 2 & 4 & 1.3827 & 8 & 19 & 6.5820 \\
\hline B4 & 11 & 11 & 11.0000 & 0.0000 & 0.00 & 153 & 167 & 25.8893 & 2 & 4 & 1.8014 & 8 & 20 & 9.8274 \\
\hline B5 & 11 & 11 & 11.0000 & 0.0000 & 0.00 & 154 & 177 & 33.0442 & 2 & 5 & 2.6769 & 9 & 29 & 17.8432 \\
\hline B6 & 7 & 7 & 7.0000 & 0.0000 & 0.00 & 154 & 165 & 11.4363 & 1 & 8 & 5.7640 & 4 & 38 & 25.6395 \\
\hline B7 & 6 & 6 & 6.0645 & 0.2497 & 0.00 & 153 & 159 & 3.8032 & 2 & 8 & 5.1941 & 9 & 36 & 22.4181 \\
\hline B8 & 4 & 4 & 4.2581 & 0.6816 & 0.00 & 152 & 158 & 5.4317 & 3 & 9 & 7.4731 & 12 & 38 & 30.7397 \\
\hline B9 & 3 & 3 & 3.0968 & 0.3005 & 0.00 & 153 & 168 & 20.1787 & 2 & 5 & 2.6102 & 10 & 25 & 11.7018 \\
\hline $\mathrm{B} 10$ & 3 & 3 & 3.0000 & 0.0000 & 0.00 & 152 & 158 & 7.1719 & 2 & 4 & 1.5794 & 8 & 17 & 6.8647 \\
\hline B11 & 4 & 4 & 4.1613 & 0.3739 & 0.00 & 154 & 162 & 8.1435 & 4 & 11 & 8.0349 & 16 & 48 & 34.9012 \\
\hline B12 & 4 & 4 & 4.0000 & 0.0000 & 0.00 & 152 & 154 & 1.6950 & 2 & 4 & 1.7064 & 8 & 18 & 6.9414 \\
\hline B13 & 4 & 4 & 4.0000 & 0.0000 & 0.00 & 152 & 154 & 2.0832 & 2 & 6 & 3.3636 & 8 & 25 & 13.6855 \\
\hline B14 & 3 & 3 & 3.4516 & 0.5680 & 0.00 & 152 & 168 & 22.8717 & 2 & 6 & 4.3793 & 8 & 27 & 19.2045 \\
\hline B15 & 1 & 1 & 1.4194 & 0.8860 & 0.00 & 153 & 175 & 34.8684 & 3 & 7 & 3.2531 & 12 & 33 & 16.1056 \\
\hline B16 & 14 & 14 & 14.0000 & 0.0000 & 0.00 & 159 & 221 & 63.7515 & 2 & 7 & 4.2848 & 11 & 41 & 21.9744 \\
\hline B17 & 13 & 13 & 13.0000 & 0.0000 & 0.00 & 154 & 178 & 48.9971 & 2 & 4 & 1.7026 & 9 & 22 & 9.6839 \\
\hline B18 & 13 & 13 & 13.0000 & 0.0000 & 0.00 & 152 & 155 & 4.3855 & 3 & 6 & 2.3655 & 12 & 24 & 10.3454 \\
\hline B19 & 13 & 13 & 13.0968 & 0.5388 & 0.00 & 152 & 153 & 2.1513 & 2 & 7 & 5.6009 & 8 & 30 & 22.7850 \\
\hline B20 & 13 & 13 & 13.0000 & 0.0000 & 0.00 & 152 & 153 & 1.5027 & 2 & 7 & 5.0298 & 8 & 32 & 20.2303 \\
\hline B21 & 9 & 9 & 9.0968 & 0.3005 & 0.00 & 154 & 158 & 3.0132 & 1 & 10 & 8.6519 & 4 & 43 & 35.6950 \\
\hline B22 & 6 & 6 & 6.1613 & 0.3739 & 0.00 & 152 & 156 & 4.0430 & 2 & 6 & 4.0364 & 8 & 26 & 16.4319 \\
\hline B23 & 6 & 6 & 6.0968 & 0.3005 & 0.00 & 152 & 153 & 1.4803 & 3 & 6 & 4.5416 & 12 & 27 & 18.2106 \\
\hline B24 & 5 & 5 & 5.3871 & 0.6152 & 0.00 & 152 & 153 & 1.5394 & 2 & 5 & 2.4132 & 8 & 23 & 9.6668 \\
\hline B25 & 4 & 4 & 4.4516 & 0.8500 & 0.00 & 152 & 154 & 1.8757 & 2 & 5 & 1.8567 & 8 & 23 & 7.7759 \\
\hline B26 & 5 & 5 & 5.0000 & 0.0000 & 0.00 & 154 & 158 & 4.2421 & 2 & 9 & 6.0213 & 8 & 40 & 25.2638 \\
\hline B27 & 3 & 3 & 3.0323 & 0.1796 & 0.00 & 152 & 156 & 3.4467 & 1 & 6 & 4.3334 & 4 & 27 & 17.5791 \\
\hline B28 & 3 & 3 & 3.3548 & 0.5507 & 0.00 & 152 & 154 & 2.4328 & 3 & 5 & 1.9724 & 12 & 24 & 8.1028 \\
\hline B29 & 3 & 3 & 3.2581 & 0.4448 & 0.00 & 152 & 154 & 3.9059 & 2 & 5 & 2.6126 & 8 & 23 & 10.6863 \\
\hline B30 & 2 & 2 & 2.2581 & 0.5143 & 0.00 & 152 & 154 & 1.8062 & 4 & 8 & 3.8077 & 16 & 32 & 15.4194 \\
\hline B31 & 7 & 7 & 7.0000 & 0.0000 & 0.00 & 154 & 166 & 12.3793 & 3 & 9 & 3.5214 & 12 & 39 & 14.8130 \\
\hline B32 & 4 & 4 & 4.1935 & 0.7492 & 0.00 & 154 & 161 & 9.6416 & 3 & 8 & 5.6009 & 12 & 35 & 23.3415 \\
\hline B33 & 4 & 4 & 4.1290 & 0.4995 & 0.00 & 152 & 160 & 7.4242 & 1 & 5 & 2.9712 & 4 & 24 & 12.6727 \\
\hline B34 & 4 & 4 & 4.0645 & 0.3592 & 0.00 & 152 & 156 & 4.2459 & 3 & 6 & 4.2170 & 12 & 28 & 17.9599 \\
\hline B35 & 4 & 4 & 4.9032 & 1.0118 & 0.00 & 153 & 166 & 10.7510 & 2 & 5 & 3.7626 & 8 & 26 & 16.9638 \\
\hline B36 & 13 & 13 & 13.0000 & 0.0000 & 0.00 & 155 & 177 & 17.3078 & 3 & 8 & 4.6900 & 12 & 38 & 23.3320 \\
\hline B37 & 10 & 10 & 10.5161 & 0.8896 & 0.00 & 153 & 165 & 14.0154 & 2 & 6 & 4.9622 & 8 & 26 & 20.6570 \\
\hline B38 & 8 & 8 & 8.0645 & 0.2497 & 0.00 & 152 & 165 & 14.2440 & 2 & 11 & 7.5177 & 8 & 52 & 33.8136 \\
\hline B39 & 5 & 5 & 5.3548 & 1.3796 & 0.00 & 154 & 168 & 10.6828 & 2 & 6 & 2.8136 & 8 & 27 & 13.6350 \\
\hline B40 & 5 & 5 & 5.1935 & 0.6011 & 0.00 & 152 & 162 & 7.7362 & 2 & 5 & 2.0832 & 8 & 22 & 8.8589 \\
\hline B41 & 8 & 8 & 8.0000 & 0.0000 & 0.00 & 154 & 177 & 24.0561 & 1 & 10 & 7.6418 & 4 & 47 & 36.2878 \\
\hline B42 & 8 & 8 & 8.0000 & 0.0000 & 0.00 & 153 & 182 & 36.9639 & 2 & 4 & 1.4081 & 8 & 20 & 10.2833 \\
\hline B43 & 8 & 8 & 8.0968 & 0.5388 & 0.00 & 162 & 268 & 137.6530 & 2 & 4 & 2.1463 & 12 & 33 & 27.5295 \\
\hline B44 & 5 & 5 & 5.8710 & 1.3842 & 0.00 & 155 & 172 & 21.8345 & 2 & 5 & 3.8857 & 8 & 27 & 19.0926 \\
\hline B45 & 5 & 5 & 5.8710 & 1.3842 & 0.00 & 153 & 182 & 50.6757 & 2 & 6 & 3.1036 & 8 & 29 & 20.3958 \\
\hline B46 & 8 & 8 & 8.1935 & 0.4016 & 0.00 & 172 & 219 & 56.1605 & 3 & 10 & 5.7329 & 15 & 60 & 43.2816 \\
\hline B47 & 5 & 5 & 5.0968 & 0.5388 & 0.00 & 166 & 207 & 40.9797 & 2 & 6 & 5.1442 & 10 & 40 & 29.2231 \\
\hline B48 & 5 & 5 & 5.0645 & 0.3592 & 0.00 & 163 & 170 & 5.2351 & 2 & 5 & 2.6258 & 8 & 26 & 12.1034 \\
\hline B49 & 5 & 5 & 5.1935 & 0.6011 & 0.00 & 160 & 170 & 8.4292 & 2 & 5 & 3.0754 & 9 & 24 & 13.6420 \\
\hline B50 & 5 & 5 & 5.5806 & 0.9228 & 0.00 & 160 & 174 & 21.8488 & 1 & 6 & 5.7528 & 4 & 28 & 25.8977 \\
\hline
\end{tabular}


Table 29: Continued.

\begin{tabular}{|c|c|c|c|c|c|c|c|c|c|c|c|c|c|c|}
\hline \multirow{2}{*}{ ID } & \multirow{2}{*}{ OPT } & \multicolumn{4}{|c|}{ Optimum } & \multicolumn{3}{|c|}{ Execution time } & \multicolumn{3}{|c|}{ Cycle } & \multicolumn{3}{|c|}{ Time cycle } \\
\hline & & $\mathrm{MBO}$ & AVG & $\mathrm{SD}$ & RPD\% & BET & AVG & ST & $\mathrm{BC}$ & AVG & $\mathrm{SD}$ & BTC & AVG & $\mathrm{SD}$ \\
\hline B51 & 27 & 27 & 28.3548 & 0.9504 & 0.00 & 167 & 198 & 36.1613 & 3 & 20 & 9.4901 & 14 & 106 & 48.8988 \\
\hline B52 & 18 & 18 & 18.7742 & 0.6688 & 0.00 & 155 & 159 & 3.0282 & 4 & 19 & 10.1538 & 17 & 84 & 42.9794 \\
\hline B53 & 11 & 11 & 11.0000 & 0.0000 & 0.00 & 154 & 159 & 4.8285 & 5 & 10 & 3.0593 & 20 & 43 & 13.3710 \\
\hline B54 & 11 & 11 & 11.0000 & 0.0000 & 0.00 & 152 & 156 & 2.9388 & 3 & 9 & 3.0061 & 12 & 38 & 12.4134 \\
\hline B55 & 7 & 7 & 7.0000 & 0.0000 & 0.00 & 154 & 164 & 12.3552 & 4 & 13 & 6.6611 & 16 & 57 & 28.3718 \\
\hline B56 & 6 & 6 & 6.0000 & 0.0000 & 0.00 & 154 & 175 & 24.3904 & 5 & 9 & 3.9809 & 22 & 44 & 17.8687 \\
\hline B57 & 6 & 6 & 6.0000 & 0.0000 & 0.00 & 153 & 163 & 6.9322 & 4 & 11 & 5.2964 & 16 & 50 & 24.4128 \\
\hline B58 & 6 & 6 & 6.0000 & 0.0000 & 0.00 & 153 & 156 & 3.5822 & 5 & 10 & 5.6569 & 21 & 41 & 23.9429 \\
\hline B59 & 9 & 9 & 9.0323 & 0.1796 & 0.00 & 155 & 166 & 5.9601 & 2 & 12 & 6.4739 & 8 & 54 & 27.6165 \\
\hline B60 & 4 & 4 & 4.5161 & 1.3631 & 0.00 & 154 & 163 & 7.2272 & 5 & 11 & 4.4119 & 21 & 49 & 19.4739 \\
\hline B61 & 4 & 4 & 4.2581 & 0.6308 & 0.00 & 154 & 158 & 6.1989 & 2 & 9 & 3.6644 & 8 & 41 & 15.6830 \\
\hline B62 & 4 & 4 & 4.1290 & 0.5623 & 0.00 & 153 & 156 & 2.4999 & 5 & 10 & 4.2704 & 20 & 42 & 17.9183 \\
\hline B63 & 27 & 27 & 27.2903 & 0.4614 & 0.00 & 155 & 166 & 6.3789 & 5 & 17 & 8.5487 & 26 & 77 & 38.2296 \\
\hline B64 & 18 & 18 & 18.2903 & 0.5287 & 0.00 & 157 & 165 & 6.0370 & 7 & 23 & 9.6062 & 28 & 104 & 42.0711 \\
\hline B65 & 14 & 14 & 14.1613 & 0.4544 & 0.00 & 154 & 160 & 4.3692 & 4 & 15 & 8.1672 & 17 & 65 & 36.0926 \\
\hline B66 & 13 & 13 & 13.0000 & 0.0000 & 0.00 & 154 & 163 & 8.8223 & 5 & 9 & 2.8923 & 20 & 42 & 12.0080 \\
\hline B67 & 11 & 11 & 11.0323 & 0.1796 & 0.00 & 159 & 170 & 7.5927 & 4 & 12 & 5.8966 & 17 & 55 & 26.7593 \\
\hline B68 & 8 & 8 & 8.5484 & 1.7481 & 0.00 & 154 & 162 & 7.1544 & 3 & 11 & 5.3146 & 12 & 50 & 22.5538 \\
\hline B69 & 8 & 8 & 8.3548 & 0.5507 & 0.00 & 153 & 156 & 2.4425 & 4 & 12 & 6.3070 & 16 & 51 & 25.7643 \\
\hline B70 & 6 & 6 & 6.2581 & 0.6308 & 0.00 & 153 & 156 & 2.2671 & 3 & 10 & 5.7856 & 12 & 44 & 23.9520 \\
\hline B71 & 6 & 6 & 6.0645 & 0.3592 & 0.00 & 156 & 181 & 27.7347 & 2 & 13 & 8.4332 & 11 & 64 & 39.5748 \\
\hline B72 & 4 & 4 & 4.1290 & 0.3408 & 0.00 & 155 & 177 & 29.3796 & 8 & 14 & 5.3225 & 36 & 64 & 23.1387 \\
\hline B73 & 4 & 4 & 4.5484 & 0.5680 & 0.00 & 153 & 182 & 46.2198 & 5 & 12 & 5.6883 & 20 & 63 & 40.8649 \\
\hline B74 & 3 & 3 & 3.4839 & 0.6768 & 0.00 & 153 & 181 & 24.0085 & 5 & 13 & 7.7974 & 22 & 62 & 38.6443 \\
\hline B75 & 11 & 11 & 11.7742 & 1.0866 & 0.00 & 164 & 216 & 65.0219 & 7 & 19 & 8.5961 & 30 & 102 & 42.8679 \\
\hline B76 & 5 & 5 & 5.1290 & 0.4995 & 0.00 & 154 & 159 & 3.2132 & 4 & 12 & 6.9587 & 19 & 50 & 28.7074 \\
\hline B77 & 5 & 5 & 5.9355 & 1.0626 & 0.00 & 154 & 159 & 2.5791 & 3 & 11 & 6.9711 & 13 & 50 & 29.2492 \\
\hline B78 & 4 & 4 & 4.7419 & 1.4135 & 0.00 & 153 & 158 & 2.8838 & 4 & 15 & 9.7257 & 20 & 64 & 40.5362 \\
\hline B79 & 14 & 14 & 14.0000 & 0.0000 & 0.00 & 156 & 162 & 5.3378 & 4 & 11 & 5.1178 & 18 & 49 & 21.6838 \\
\hline B80 & 11 & 11 & 11.0000 & 0.0000 & 0.00 & 153 & 159 & 2.9928 & 4 & 10 & 3.3575 & 16 & 46 & 14.4630 \\
\hline B81 & 11 & 11 & 11.2903 & 1.0064 & 0.00 & 153 & 158 & 2.8994 & 5 & 12 & 5.9435 & 21 & 52 & 25.2294 \\
\hline B82 & 10 & 10 & 10.6129 & 0.9892 & 0.00 & 154 & 158 & 2.6680 & 2 & 10 & 5.5058 & 8 & 45 & 22.8974 \\
\hline B83 & 12 & 12 & 12.0000 & 0.0000 & 0.00 & 156 & 165 & 6.5898 & 4 & 10 & 3.6210 & 20 & 46 & 14.5889 \\
\hline B84 & 12 & 12 & 12.0645 & 0.3592 & 0.00 & 155 & 163 & 5.1148 & 4 & 10 & 5.6932 & 18 & 44 & 24.3315 \\
\hline B85 & 8 & 8 & 8.9355 & 1.2893 & 0.00 & 155 & 161 & 5.5007 & 4 & 16 & 8.2380 & 16 & 69 & 35.8578 \\
\hline B86 & 8 & 8 & 8.0645 & 0.2497 & 0.00 & 153 & 165 & 10.2890 & 3 & 10 & 4.3411 & 15 & 46 & 18.2102 \\
\hline B87 & 10 & 10 & 10.5484 & 0.9605 & 0.00 & 157 & 177 & 13.8084 & 5 & 18 & 7.6776 & 23 & 87 & 37.4388 \\
\hline B88 & 8 & 8 & 8.2581 & 1.4368 & 0.00 & 154 & 166 & 13.5674 & 5 & 10 & 5.8803 & 24 & 46 & 24.4021 \\
\hline B89 & 8 & 8 & 8.0323 & 0.1796 & 0.00 & 156 & 168 & 18.2189 & 6 & 9 & 3.7301 & 24 & 43 & 20.6299 \\
\hline \multirow[t]{2}{*}{ B90 } & 5 & 5 & 5.3871 & 1.0223 & 0.00 & 153 & 164 & 9.5021 & 4 & 12 & 5.7030 & 18 & 54 & 24.8741 \\
\hline & & AVG & 8.1115 & 0.4667 & 0.00 & 154 & 167 & 15.5926 & 3 & 9 & 4.8703 & 13 & 42 & 22.3487 \\
\hline
\end{tabular}


TABLE 30: MBO using parallel quick sort algorithm with 4 threads.

\begin{tabular}{|c|c|c|c|c|c|c|c|c|c|c|c|c|c|c|}
\hline \multirow{2}{*}{ ID } & \multirow{2}{*}{ OPT } & \multicolumn{4}{|c|}{ Optimum } & \multicolumn{3}{|c|}{ Execution time } & \multicolumn{3}{|c|}{ Cycle } & \multicolumn{3}{|c|}{ Time cycle } \\
\hline & & $\mathrm{MBO}$ & AVG & SD & RPD\% & BET & AVG & ST & $\mathrm{BC}$ & AVG & SD & BTC & AVG & $\mathrm{SD}$ \\
\hline B1 & 11 & 11 & 11.0000 & 0.0000 & 0.00 & 154 & 188 & 70.6184 & 1 & 6 & 4.1450 & 7 & 35 & 21.4910 \\
\hline B2 & 11 & 11 & 11.0000 & 0.0000 & 0.00 & 152 & 182 & 34.7551 & 2 & 3 & 1.4591 & 8 & 19 & 11.0777 \\
\hline B3 & 11 & 11 & 11.0000 & 0.0000 & 0.00 & 153 & 177 & 33.6228 & 2 & 4 & 1.7083 & 8 & 19 & 7.7936 \\
\hline B4 & 11 & 11 & 11.0000 & 0.0000 & 0.00 & 153 & 170 & 28.4944 & 2 & 4 & 1.7314 & 8 & 21 & 7.5054 \\
\hline B5 & 11 & 11 & 11.0000 & 0.0000 & 0.00 & 153 & 177 & 33.1479 & 2 & 4 & 2.1347 & 8 & 23 & 13.0829 \\
\hline B6 & 7 & 7 & 7.0000 & 0.0000 & 0.00 & 161 & 192 & 19.0742 & 3 & 9 & 5.1148 & 15 & 50 & 25.7463 \\
\hline B7 & 6 & 6 & 6.0323 & 0.1796 & 0.00 & 152 & 159 & 9.1029 & 1 & 5 & 2.5413 & 4 & 23 & 12.2419 \\
\hline B8 & 4 & 4 & 4.4516 & 1.2339 & 0.00 & 152 & 159 & 12.7028 & 2 & 11 & 8.2441 & 8 & 46 & 33.8427 \\
\hline B9 & 3 & 3 & 3.0968 & 0.3005 & 0.00 & 158 & 257 & 84.1696 & 1 & 6 & 3.5033 & 14 & 44 & 31.5700 \\
\hline B10 & 3 & 3 & 3.0000 & 0.0000 & 0.00 & 153 & 183 & 28.7203 & 2 & 4 & 2.5145 & 8 & 24 & 18.5823 \\
\hline B11 & 4 & 4 & 4.0968 & 0.3005 & 0.00 & 156 & 165 & 7.6151 & 3 & 11 & 6.1848 & 13 & 50 & 27.3824 \\
\hline B12 & 4 & 4 & 4.0000 & 0.0000 & 0.00 & 152 & 155 & 2.0000 & 2 & 4 & 1.5596 & 8 & 16 & 6.3920 \\
\hline B13 & 4 & 4 & 4.0645 & 0.3592 & 0.00 & 152 & 154 & 1.8538 & 3 & 4 & 2.1089 & 12 & 19 & 8.8271 \\
\hline B14 & 3 & 3 & 3.2903 & 0.4614 & 0.00 & 152 & 160 & 9.8399 & 1 & 5 & 2.5222 & 4 & 24 & 10.5106 \\
\hline B15 & 1 & 1 & 1.4516 & 0.8500 & 0.00 & 152 & 156 & 3.8373 & 1 & 5 & 3.5542 & 4 & 24 & 14.7010 \\
\hline B16 & 14 & 14 & 14.0000 & 0.0000 & 0.00 & 154 & 173 & 25.6756 & 3 & 9 & 4.8887 & 12 & 41 & 27.1373 \\
\hline B17 & 13 & 13 & 13.0000 & 0.0000 & 0.00 & 153 & 157 & 6.4014 & 2 & 5 & 1.6950 & 8 & 21 & 7.1785 \\
\hline B18 & 13 & 13 & 13.0000 & 0.0000 & 0.00 & 152 & 155 & 5.7326 & 2 & 5 & 2.4926 & 8 & 22 & 10.7346 \\
\hline B19 & 13 & 13 & 13.0000 & 0.0000 & 0.00 & 152 & 157 & 7.7789 & 3 & 7 & 3.6761 & 12 & 30 & 15.0969 \\
\hline B20 & 13 & 13 & 13.0000 & 0.0000 & 0.00 & 152 & 155 & 4.7308 & 2 & 6 & 3.0810 & 8 & 27 & 12.7058 \\
\hline B21 & 9 & 9 & 9.0968 & 0.3005 & 0.00 & 154 & 158 & 2.9072 & 3 & 11 & 7.1601 & 12 & 46 & 30.0206 \\
\hline B22 & 6 & 6 & 6.0645 & 0.2497 & 0.00 & 154 & 182 & 76.4377 & 3 & 6 & 2.7909 & 12 & 39 & 63.2356 \\
\hline B23 & 6 & 6 & 6.1290 & 0.4275 & 0.00 & 152 & 160 & 13.7304 & 2 & 7 & 4.4337 & 8 & 33 & 23.9618 \\
\hline B24 & 5 & 5 & 5.3871 & 0.6672 & 0.00 & 152 & 154 & 2.6893 & 3 & 6 & 2.5123 & 12 & 27 & 10.0762 \\
\hline B25 & 4 & 4 & 4.1935 & 0.6011 & 0.00 & 152 & 155 & 3.2505 & 2 & 6 & 2.8128 & 10 & 26 & 11.2697 \\
\hline B26 & 5 & 5 & 5.0000 & 0.0000 & 0.00 & 153 & 158 & 4.2021 & 2 & 8 & 6.8480 & 8 & 37 & 28.5317 \\
\hline B27 & 3 & 3 & 3.0968 & 0.3005 & 0.00 & 152 & 159 & 7.4229 & 3 & 8 & 6.4966 & 12 & 34 & 27.3041 \\
\hline B28 & 3 & 3 & 3.1935 & 0.4016 & 0.00 & 152 & 174 & 36.7644 & 2 & 6 & 4.5661 & 10 & 30 & 20.8310 \\
\hline B29 & 3 & 3 & 3.2258 & 0.4250 & 0.00 & 152 & 154 & 1.6224 & 1 & 5 & 1.8526 & 4 & 20 & 7.7248 \\
\hline B30 & 2 & 2 & 2.3871 & 0.5584 & 0.00 & 152 & 153 & 2.0651 & 2 & 6 & 3.0268 & 8 & 25 & 12.0320 \\
\hline B31 & 7 & 7 & 7.0000 & 0.0000 & 0.00 & 154 & 158 & 3.8702 & 2 & 9 & 5.1157 & 8 & 40 & 21.2321 \\
\hline B32 & 4 & 4 & 4.0000 & 0.0000 & 0.00 & 153 & 155 & 2.0925 & 2 & 6 & 3.6732 & 8 & 27 & 15.0795 \\
\hline B33 & 4 & 4 & 4.0645 & 0.3592 & 0.00 & 152 & 154 & 2.3150 & 1 & 5 & 2.5089 & 4 & 24 & 10.0413 \\
\hline B34 & 4 & 4 & 4.2581 & 0.6816 & 0.00 & 152 & 154 & 3.8401 & 2 & 6 & 4.1587 & 8 & 28 & 17.4530 \\
\hline B35 & 4 & 4 & 4.7097 & 0.9727 & 0.00 & 152 & 153 & 1.4701 & 1 & 7 & 5.4404 & 4 & 29 & 21.7851 \\
\hline B36 & 13 & 13 & 13.0000 & 0.0000 & 0.00 & 154 & 157 & 3.1167 & 2 & 6 & 3.5128 & 8 & 29 & 14.5407 \\
\hline B37 & 10 & 10 & 10.4516 & 0.8500 & 0.00 & 152 & 155 & 2.1840 & 1 & 5 & 3.6124 & 4 & 24 & 14.6059 \\
\hline B38 & 8 & 8 & 8.0323 & 0.1796 & 0.00 & 152 & 155 & 3.6706 & 2 & 14 & 9.2075 & 8 & 59 & 37.5165 \\
\hline B39 & 5 & 5 & 5.1613 & 0.8980 & 0.00 & 152 & 153 & 1.7339 & 2 & 5 & 2.6929 & 8 & 22 & 10.9139 \\
\hline B40 & 5 & 5 & 5.2581 & 0.6816 & 0.00 & 152 & 153 & 1.6091 & 2 & 5 & 2.4279 & 8 & 23 & 10.0457 \\
\hline B41 & 8 & 8 & 8.0000 & 0.0000 & 0.00 & 154 & 158 & 4.7956 & 2 & 7 & 4.4959 & 8 & 32 & 18.6759 \\
\hline B42 & 8 & 8 & 8.0000 & 0.0000 & 0.00 & 152 & 154 & 1.7469 & 2 & 4 & 2.2418 & 8 & 17 & 9.4866 \\
\hline B43 & 8 & 8 & 8.0000 & 0.0000 & 0.00 & 152 & 154 & 2.0064 & 1 & 5 & 2.4552 & 4 & 21 & 10.1844 \\
\hline B44 & 5 & 5 & 6.2581 & 1.5048 & 0.00 & 152 & 153 & 1.3647 & 1 & 6 & 3.6715 & 4 & 25 & 14.7284 \\
\hline B45 & 5 & 5 & 6.2903 & 1.5534 & 0.00 & 152 & 154 & 3.6805 & 3 & 5 & 2.3316 & 12 & 22 & 9.3036 \\
\hline B46 & 8 & 8 & 8.1290 & 0.3408 & 0.00 & 154 & 158 & 2.9719 & 3 & 9 & 6.7561 & 12 & 39 & 28.1927 \\
\hline B47 & 5 & 5 & 5.0968 & 0.5388 & 0.00 & 153 & 165 & 8.1313 & 2 & 5 & 2.2322 & 9 & 22 & 9.6494 \\
\hline B48 & 5 & 5 & 5.1935 & 0.6011 & 0.00 & 156 & 169 & 11.8787 & 1 & 6 & 4.2139 & 4 & 27 & 18.5138 \\
\hline B49 & 5 & 5 & 5.2581 & 0.6816 & 0.00 & 152 & 182 & 30.8552 & 2 & 5 & 2.3787 & 10 & 24 & 10.4171 \\
\hline B50 & 5 & 5 & 5.2581 & 0.6816 & 0.00 & 152 & 196 & 63.4998 & 3 & 8 & 5.8501 & 12 & 40 & 28.8074 \\
\hline
\end{tabular}


TABle 30: Continued.

\begin{tabular}{|c|c|c|c|c|c|c|c|c|c|c|c|c|c|c|}
\hline \multirow{2}{*}{ ID } & \multirow{2}{*}{ OPT } & \multicolumn{4}{|c|}{ Optimum } & \multicolumn{3}{|c|}{ Execution time } & \multicolumn{3}{|c|}{ Cycle } & \multicolumn{3}{|c|}{ Time cycle } \\
\hline & & $\mathrm{MBO}$ & AVG & $\mathrm{SD}$ & RPD\% & BET & AVG & ST & $\mathrm{BC}$ & AVG & $\mathrm{SD}$ & BTC & AVG & SD \\
\hline B51 & 27 & 27 & 28.2581 & 1.2902 & 0.00 & 154 & 167 & 15.4226 & 7 & 21 & 7.9024 & 29 & 95 & 36.2584 \\
\hline B52 & 18 & 18 & 18.5806 & 0.9228 & 0.00 & 154 & 157 & 4.2457 & 7 & 20 & 9.5797 & 29 & 85 & 41.1011 \\
\hline B53 & 11 & 11 & 11.0000 & 0.0000 & 0.00 & 154 & 156 & 2.3477 & 5 & 10 & 3.7096 & 20 & 41 & 15.1854 \\
\hline B54 & 11 & 11 & 11.0000 & 0.0000 & 0.00 & 152 & 154 & 2.3408 & 6 & 9 & 2.1973 & 24 & 37 & 9.2985 \\
\hline B55 & 7 & 7 & 7.0000 & 0.0000 & 0.00 & 154 & 157 & 2.9607 & 4 & 11 & 4.6251 & 20 & 48 & 20.0461 \\
\hline B56 & 6 & 6 & 6.0000 & 0.0000 & 0.00 & 154 & 156 & 3.9259 & 5 & 10 & 3.7273 & 20 & 44 & 15.4734 \\
\hline B57 & 6 & 6 & 6.1290 & 0.4995 & 0.00 & 152 & 155 & 1.9394 & 4 & 11 & 3.9784 & 16 & 48 & 16.3756 \\
\hline B58 & 6 & 6 & 6.0000 & 0.0000 & 0.00 & 153 & 155 & 1.8439 & 4 & 9 & 3.4241 & 16 & 39 & 13.8646 \\
\hline B59 & 9 & 9 & 9.1290 & 0.4275 & 0.00 & 154 & 158 & 5.4091 & 4 & 11 & 4.9422 & 19 & 49 & 20.9249 \\
\hline B60 & 4 & 4 & 4.6452 & 1.4955 & 0.00 & 153 & 156 & 2.4622 & 5 & 12 & 6.4630 & 21 & 52 & 27.5544 \\
\hline B61 & 4 & 4 & 4.0323 & 0.1796 & 0.00 & 153 & 156 & 2.9838 & 3 & 11 & 7.3924 & 12 & 46 & 30.1892 \\
\hline B62 & 4 & 4 & 4.0323 & 0.1796 & 0.00 & 153 & 165 & 11.7614 & 3 & 9 & 4.1137 & 12 & 41 & 18.7758 \\
\hline B63 & 27 & 27 & 27.1935 & 0.4016 & 0.00 & 155 & 174 & 17.4625 & 6 & 17 & 9.3253 & 25 & 77 & 41.1960 \\
\hline B64 & 18 & 18 & 18.2903 & 0.5287 & 0.00 & 154 & 168 & 12.6685 & 4 & 18 & 9.1350 & 16 & 81 & 44.2824 \\
\hline B65 & 14 & 14 & 14.0323 & 0.1796 & 0.00 & 155 & 167 & 9.6409 & 3 & 15 & 7.6449 & 15 & 69 & 33.6520 \\
\hline B66 & 13 & 13 & 13.0000 & 0.0000 & 0.00 & 153 & 160 & 6.3507 & 4 & 9 & 3.0157 & 19 & 42 & 13.7272 \\
\hline B67 & 11 & 11 & 11.0000 & 0.0000 & 0.00 & 155 & 175 & 15.9737 & 6 & 15 & 8.3154 & 26 & 73 & 40.7381 \\
\hline B68 & 8 & 8 & 8.3226 & 1.2751 & 0.00 & 153 & 164 & 16.0762 & 3 & 11 & 6.8135 & 13 & 52 & 31.3006 \\
\hline B69 & 8 & 8 & 8.4839 & 0.6768 & 0.00 & 153 & 157 & 4.3202 & 4 & 12 & 6.9641 & 17 & 53 & 28.7297 \\
\hline B70 & 6 & 6 & 6.6452 & 0.9504 & 0.00 & 152 & 155 & 4.3401 & 4 & 15 & 8.3663 & 16 & 65 & 33.8478 \\
\hline B71 & 6 & 6 & 6.0645 & 0.3592 & 0.00 & 154 & 158 & 3.7631 & 7 & 13 & 6.9158 & 30 & 58 & 28.7037 \\
\hline B72 & 4 & 4 & 4.3226 & 0.6525 & 0.00 & 153 & 156 & 2.3215 & 4 & 13 & 8.2617 & 16 & 55 & 33.7554 \\
\hline B73 & 4 & 4 & 4.6774 & 0.6525 & 0.00 & 153 & 159 & 6.6099 & 3 & 11 & 5.8756 & 12 & 48 & 25.2215 \\
\hline B74 & 3 & 3 & 3.5484 & 0.5680 & 0.00 & 152 & 158 & 6.5660 & 4 & 13 & 7.7735 & 17 & 54 & 32.1404 \\
\hline B75 & 11 & 11 & 11.7419 & 1.0945 & 0.00 & 155 & 162 & 9.8424 & 5 & 18 & 7.4919 & 21 & 77 & 31.6711 \\
\hline B76 & 5 & 5 & 5.1935 & 0.6011 & 0.00 & 154 & 169 & 17.1585 & 6 & 14 & 6.6203 & 26 & 64 & 36.1670 \\
\hline B77 & 5 & 5 & 5.5161 & 0.8896 & 0.00 & 153 & 166 & 17.0023 & 3 & 13 & 6.6431 & 14 & 56 & 28.0002 \\
\hline B78 & 4 & 4 & 4.8387 & 1.4628 & 0.00 & 154 & 167 & 16.8144 & 4 & 17 & 8.5824 & 18 & 77 & 36.9169 \\
\hline B79 & 14 & 14 & 14.0000 & 0.0000 & 0.00 & 158 & 182 & 31.2523 & 4 & 9 & 6.0580 & 20 & 48 & 28.0606 \\
\hline B80 & 11 & 11 & 11.2581 & 1.4368 & 0.00 & 154 & 168 & 12.6654 & 4 & 11 & 4.0099 & 16 & 51 & 21.3734 \\
\hline B81 & 11 & 11 & 11.3871 & 0.9549 & 0.00 & 153 & 164 & 10.3919 & 4 & 15 & 9.5243 & 19 & 67 & 42.0640 \\
\hline B82 & 10 & 10 & 10.6452 & 0.7094 & 0.00 & 153 & 160 & 9.4966 & 5 & 11 & 5.4876 & 23 & 51 & 27.6218 \\
\hline B83 & 12 & 12 & 12.0000 & 0.0000 & 0.00 & 154 & 157 & 2.8341 & 4 & 12 & 4.8057 & 19 & 51 & 19.9222 \\
\hline B84 & 12 & 12 & 12.2903 & 0.7829 & 0.00 & 153 & 157 & 5.0586 & 5 & 14 & 8.1952 & 20 & 59 & 34.1199 \\
\hline B85 & 8 & 8 & 8.9677 & 1.3536 & 0.00 & 154 & 162 & 10.7207 & 8 & 15 & 6.6079 & 33 & 65 & 31.6611 \\
\hline B86 & 8 & 8 & 8.0323 & 0.1796 & 0.00 & 152 & 166 & 10.2565 & 4 & 9 & 3.7700 & 16 & 40 & 17.8689 \\
\hline B87 & 10 & 10 & 10.7097 & 0.9727 & 0.00 & 154 & 166 & 10.2930 & 7 & 19 & 8.5170 & 28 & 82 & 37.4828 \\
\hline B88 & 8 & 8 & 8.0000 & 0.0000 & 0.00 & 160 & 177 & 8.3988 & 4 & 10 & 4.3527 & 18 & 50 & 20.4859 \\
\hline B89 & 8 & 8 & 8.0323 & 0.1796 & 0.00 & 154 & 173 & 15.7080 & 2 & 10 & 8.1272 & 8 & 49 & 40.5965 \\
\hline \multirow[t]{2}{*}{ B90 } & 5 & 5 & 5.2903 & 0.9016 & 0.00 & 154 & 164 & 9.5502 & 4 & 11 & 5.5830 & 16 & 49 & 26.4783 \\
\hline & & AVG & 8.1108 & 0.4544 & 0.00 & 153 & 163 & 12.2109 & 3 & 9 & 4.9019 & 13 & 41 & 22.4733 \\
\hline
\end{tabular}


TABLE 31: MBO using parallel quick sort algorithm with 8 threads.

\begin{tabular}{|c|c|c|c|c|c|c|c|c|c|c|c|c|c|c|}
\hline \multirow{2}{*}{ ID } & \multirow{2}{*}{ OPT } & \multicolumn{4}{|c|}{ Optimum } & \multicolumn{3}{|c|}{ Execution time } & \multicolumn{3}{|c|}{ Cycle } & \multicolumn{3}{|c|}{ Time cycle } \\
\hline & & $\mathrm{MBO}$ & AVG & $\mathrm{SD}$ & RPD\% & BET & AVG & ST & $\mathrm{BC}$ & AVG & $\mathrm{SD}$ & BTC & AVG & SD \\
\hline B1 & 11 & 11 & 11.0000 & 0.0000 & 0.00 & 190 & 260 & 90.7097 & 2 & 6 & 3.5636 & 12 & 55 & 39.8897 \\
\hline B2 & 11 & 11 & 11.0000 & 0.0000 & 0.00 & 205 & 231 & 16.0884 & 1 & 3 & 1.3408 & 5 & 27 & 11.3853 \\
\hline B3 & 11 & 11 & 11.0000 & 0.0000 & 0.00 & 201 & 218 & 12.8483 & 2 & 4 & 1.2487 & 15 & 25 & 8.4055 \\
\hline B4 & 11 & 11 & 11.0000 & 0.0000 & 0.00 & 197 & 224 & 13.2765 & 2 & 4 & 1.6198 & 9 & 30 & 10.1960 \\
\hline B5 & 11 & 11 & 11.0000 & 0.0000 & 0.00 & 203 & 226 & 16.9506 & 1 & 5 & 2.5425 & 5 & 31 & 15.3820 \\
\hline B6 & 7 & 7 & 7.0000 & 0.0000 & 0.00 & 179 & 209 & 26.6540 & 3 & 8 & 4.1551 & 16 & 46 & 20.1586 \\
\hline B7 & 6 & 6 & 6.0645 & 0.2497 & 0.00 & 159 & 170 & 8.6506 & 2 & 5 & 2.3958 & 9 & 26 & 10.8302 \\
\hline B8 & 4 & 4 & 4.5806 & 1.2852 & 0.00 & 161 & 208 & 24.9863 & 2 & 9 & 8.2168 & 11 & 49 & 44.8206 \\
\hline B9 & 3 & 3 & 3.0000 & 0.0000 & 0.00 & 156 & 169 & 9.0874 & 1 & 5 & 2.9975 & 4 & 25 & 14.9742 \\
\hline B10 & 3 & 3 & 3.0000 & 0.0000 & 0.00 & 160 & 187 & 29.3461 & 1 & 4 & 1.9143 & 4 & 21 & 10.0919 \\
\hline B11 & 4 & 4 & 4.2581 & 0.4448 & 0.00 & 221 & 265 & 38.7226 & 3 & 12 & 7.1297 & 18 & 92 & 57.9354 \\
\hline B12 & 4 & 4 & 4.0000 & 0.0000 & 0.00 & 189 & 244 & 25.8799 & 2 & 4 & 1.8917 & 9 & 30 & 13.7061 \\
\hline B13 & 4 & 4 & 4.0000 & 0.0000 & 0.00 & 175 & 212 & 26.0266 & 2 & 5 & 2.0609 & 9 & 29 & 12.7301 \\
\hline B14 & 3 & 3 & 3.5161 & 0.5080 & 0.00 & 191 & 233 & 25.7782 & 2 & 5 & 2.1251 & 10 & 32 & 17.8553 \\
\hline B15 & 1 & 1 & 1.4839 & 0.9263 & 0.00 & 158 & 172 & 15.6618 & 2 & 5 & 2.1266 & 8 & 25 & 10.8872 \\
\hline B16 & 14 & 14 & 14.0000 & 0.0000 & 0.00 & 219 & 241 & 17.5973 & 2 & 8 & 4.4454 & 10 & 51 & 28.6909 \\
\hline B17 & 13 & 13 & 13.0000 & 0.0000 & 0.00 & 155 & 178 & 22.8931 & 2 & 5 & 1.4142 & 8 & 23 & 6.1196 \\
\hline B18 & 13 & 13 & 13.0000 & 0.0000 & 0.00 & 157 & 170 & 16.5604 & 2 & 5 & 1.9620 & 8 & 23 & 10.1844 \\
\hline B19 & 13 & 13 & 13.2903 & 0.9016 & 0.00 & 184 & 212 & 13.5299 & 2 & 6 & 2.9024 & 10 & 38 & 15.5537 \\
\hline B20 & 13 & 13 & 13.0000 & 0.0000 & 0.00 & 179 & 195 & 11.0056 & 2 & 8 & 5.7083 & 8 & 44 & 29.5659 \\
\hline B21 & 9 & 9 & 9.1613 & 0.3739 & 0.00 & 173 & 223 & 67.1284 & 4 & 10 & 8.0616 & 19 & 60 & 50.9887 \\
\hline B22 & 6 & 6 & 6.1613 & 0.3739 & 0.00 & 193 & 273 & 88.1693 & 3 & 6 & 3.0764 & 12 & 46 & 37.1433 \\
\hline B23 & 6 & 6 & 6.2258 & 0.4973 & 0.00 & 187 & 232 & 20.6745 & 2 & 6 & 4.7992 & 14 & 44 & 30.3885 \\
\hline B24 & 5 & 5 & 5.4516 & 0.7229 & 0.00 & 185 & 216 & 28.3318 & 2 & 5 & 2.5046 & 14 & 32 & 12.6408 \\
\hline B25 & 4 & 4 & 4.3548 & 0.7094 & 0.00 & 180 & 220 & 38.7354 & 2 & 7 & 3.3575 & 11 & 41 & 21.8570 \\
\hline B26 & 5 & 5 & 5.0000 & 0.0000 & 0.00 & 222 & 267 & 65.9248 & 2 & 9 & 6.2550 & 11 & 63 & 40.3980 \\
\hline B27 & 3 & 3 & 3.0968 & 0.3005 & 0.00 & 163 & 187 & 72.6372 & 1 & 6 & 4.0640 & 4 & 32 & 19.3312 \\
\hline B28 & 3 & 3 & 3.2903 & 0.4614 & 0.00 & 161 & 188 & 57.0519 & 2 & 5 & 2.3773 & 8 & 26 & 13.4644 \\
\hline B29 & 3 & 3 & 3.1290 & 0.3408 & 0.00 & 153 & 176 & 30.2510 & 2 & 5 & 1.9615 & 9 & 24 & 10.0626 \\
\hline B30 & 2 & 2 & 2.3871 & 0.5584 & 0.00 & 153 & 175 & 21.4547 & 3 & 9 & 6.7079 & 12 & 41 & 29.4979 \\
\hline B31 & 7 & 7 & 7.0000 & 0.0000 & 0.00 & 161 & 211 & 43.1659 & 2 & 10 & 4.5541 & 10 & 58 & 30.4089 \\
\hline B32 & 4 & 4 & 4.3871 & 1.0223 & 0.00 & 155 & 191 & 40.5896 & 1 & 7 & 6.1083 & 4 & 38 & 31.5310 \\
\hline B33 & 4 & 4 & 4.1935 & 0.6011 & 0.00 & 156 & 214 & 47.1663 & 2 & 6 & 3.0204 & 12 & 37 & 18.9796 \\
\hline B34 & 4 & 4 & 4.2581 & 0.6816 & 0.00 & 156 & 169 & 21.7325 & 2 & 5 & 2.2855 & 10 & 26 & 10.2277 \\
\hline B35 & 4 & 4 & 4.4516 & 0.8500 & 0.00 & 155 & 184 & 36.3288 & 1 & 5 & 2.4861 & 4 & 25 & 14.0484 \\
\hline B36 & 13 & 13 & 13.0000 & 0.0000 & 0.00 & 159 & 172 & 12.9491 & 2 & 9 & 5.4378 & 8 & 44 & 24.1031 \\
\hline B37 & 10 & 10 & 10.5806 & 0.9228 & 0.00 & 154 & 165 & 12.0246 & 2 & 7 & 6.6159 & 9 & 35 & 31.7865 \\
\hline B38 & 8 & 8 & 8.0968 & 0.3005 & 0.00 & 155 & 162 & 8.0036 & 2 & 11 & 9.7551 & 8 & 50 & 41.9069 \\
\hline B39 & 5 & 5 & 5.0000 & 0.0000 & 0.00 & 154 & 162 & 6.2843 & 2 & 5 & 2.1109 & 8 & 23 & 9.1885 \\
\hline B40 & 5 & 5 & 5.1290 & 0.4995 & 0.00 & 160 & 185 & 31.2620 & 2 & 5 & 1.9898 & 9 & 27 & 11.3643 \\
\hline B41 & 8 & 8 & 8.1613 & 0.8980 & 0.00 & 157 & 191 & 35.3240 & 3 & 9 & 5.6883 & 12 & 52 & 34.1054 \\
\hline B42 & 8 & 8 & 8.0000 & 0.0000 & 0.00 & 153 & 191 & 35.1184 & 2 & 4 & 2.0557 & 8 & 25 & 14.0413 \\
\hline B43 & 8 & 8 & 8.0000 & 0.0000 & 0.00 & 153 & 195 & 46.0170 & 2 & 4 & 1.8163 & 8 & 26 & 13.6305 \\
\hline B44 & 5 & 5 & 5.9677 & 1.4256 & 0.00 & 155 & 180 & 30.1549 & 1 & 5 & 2.3372 & 4 & 23 & 10.0189 \\
\hline B45 & 5 & 5 & 5.8710 & 1.3842 & 0.00 & 154 & 162 & 10.2862 & 1 & 5 & 2.5661 & 4 & 24 & 11.2124 \\
\hline B46 & 8 & 8 & 8.0645 & 0.2497 & 0.00 & 159 & 197 & 41.1302 & 2 & 10 & 6.8400 & 8 & 53 & 42.0245 \\
\hline B47 & 5 & 5 & 5.0968 & 0.5388 & 0.00 & 156 & 185 & 49.5067 & 2 & 6 & 2.5703 & 9 & 34 & 21.4733 \\
\hline B48 & 5 & 5 & 5.1290 & 0.4995 & 0.00 & 154 & 183 & 34.0827 & 2 & 5 & 2.5493 & 9 & 28 & 13.3359 \\
\hline B49 & 5 & 5 & 5.2581 & 0.6816 & 0.00 & 155 & 171 & 26.4985 & 2 & 6 & 4.9605 & 8 & 27 & 22.1096 \\
\hline B50 & 5 & 5 & 5.1935 & 0.6011 & 0.00 & 159 & 293 & 137.7965 & 2 & 6 & 4.3729 & 10 & 44 & 30.5390 \\
\hline
\end{tabular}


TABLE 31: Continued.

\begin{tabular}{|c|c|c|c|c|c|c|c|c|c|c|c|c|c|c|}
\hline \multirow{2}{*}{ ID } & \multirow{2}{*}{ OPT } & \multicolumn{4}{|c|}{ Optimum } & \multicolumn{3}{|c|}{ Execution time } & \multicolumn{3}{|c|}{ Cycle } & \multicolumn{3}{|c|}{ Time cycle } \\
\hline & & $\mathrm{MBO}$ & AVG & $\mathrm{SD}$ & RPD\% & BET & AVG & ST & $\mathrm{BC}$ & AVG & $\mathrm{SD}$ & BTC & AVG & SD \\
\hline B51 & 27 & 27 & 28.2903 & 0.8638 & 0.00 & 237 & 261 & 14.2311 & 4 & 21 & 9.5117 & 22 & 144 & 67.3566 \\
\hline B52 & 18 & 18 & 18.7742 & 0.8835 & 0.00 & 219 & 233 & 14.9013 & 3 & 21 & 10.1319 & 19 & 133 & 65.4978 \\
\hline B53 & 11 & 11 & 11.0000 & 0.0000 & 0.00 & 201 & 230 & 12.1596 & 5 & 11 & 3.8566 & 28 & 66 & 25.8455 \\
\hline B54 & 11 & 11 & 11.0000 & 0.0000 & 0.00 & 202 & 217 & 12.3461 & 3 & 8 & 2.6401 & 21 & 48 & 16.3395 \\
\hline B55 & 7 & 7 & 7.0000 & 0.0000 & 0.00 & 214 & 240 & 12.0357 & 4 & 11 & 4.1032 & 23 & 74 & 26.0514 \\
\hline B56 & 6 & 6 & 6.0000 & 0.0000 & 0.00 & 204 & 224 & 12.4441 & 4 & 11 & 4.5166 & 22 & 66 & 28.6479 \\
\hline B57 & 6 & 6 & 6.0323 & 0.1796 & 0.00 & 208 & 228 & 12.0374 & 4 & 11 & 3.5765 & 24 & 67 & 21.0756 \\
\hline B58 & 6 & 6 & 6.0000 & 0.0000 & 0.00 & 206 & 224 & 11.6898 & 4 & 9 & 4.5009 & 16 & 56 & 28.0782 \\
\hline B59 & 9 & 9 & 9.1290 & 0.4275 & 0.00 & 174 & 241 & 39.2589 & 5 & 16 & 9.8178 & 23 & 103 & 60.2449 \\
\hline B60 & 4 & 4 & 4.7419 & 1.7883 & 0.00 & 154 & 159 & 3.9053 & 3 & 12 & 5.6472 & 12 & 51 & 24.0830 \\
\hline B61 & 4 & 4 & 4.1613 & 0.3739 & 0.00 & 154 & 157 & 4.0611 & 3 & 12 & 7.1637 & 12 & 51 & 29.2026 \\
\hline B62 & 4 & 4 & 4.0000 & 0.0000 & 0.00 & 154 & 157 & 4.8422 & 4 & 10 & 5.3876 & 17 & 43 & 22.6520 \\
\hline B63 & 27 & 27 & 27.1935 & 0.4016 & 0.00 & 158 & 169 & 5.2271 & 3 & 14 & 7.9795 & 14 & 66 & 35.6641 \\
\hline B64 & 18 & 18 & 18.2903 & 0.6426 & 0.00 & 155 & 159 & 2.8364 & 3 & 19 & 10.2648 & 14 & 84 & 42.7305 \\
\hline B65 & 14 & 14 & 14.0968 & 0.3005 & 0.00 & 154 & 163 & 8.1524 & 3 & 14 & 7.8763 & 13 & 62 & 35.1641 \\
\hline B66 & 13 & 13 & 13.0000 & 0.0000 & 0.00 & 154 & 166 & 13.0996 & 7 & 12 & 3.6136 & 28 & 54 & 17.3759 \\
\hline B67 & 11 & 11 & 11.0968 & 0.3005 & 0.00 & 166 & 263 & 83.3463 & 3 & 15 & 9.6607 & 17 & 109 & 100.7377 \\
\hline B68 & 8 & 8 & 8.1935 & 0.6011 & 0.00 & 155 & 184 & 23.4952 & 4 & 12 & 6.7003 & 19 & 60 & 35.4280 \\
\hline B69 & 8 & 8 & 8.5806 & 0.7199 & 0.00 & 158 & 211 & 64.9205 & 5 & 12 & 6.2117 & 22 & 75 & 65.2722 \\
\hline B70 & 6 & 6 & 6.7097 & 0.8638 & 0.00 & 155 & 231 & 135.2276 & 3 & 13 & 6.6360 & 18 & 83 & 66.1519 \\
\hline B71 & 6 & 6 & 6.2258 & 0.6170 & 0.00 & 162 & 200 & 53.9548 & 5 & 12 & 8.4985 & 27 & 67 & 46.9196 \\
\hline B72 & 4 & 4 & 4.3226 & 0.5993 & 0.00 & 154 & 160 & 4.7503 & 6 & 12 & 5.8701 & 24 & 53 & 24.2337 \\
\hline B73 & 4 & 4 & 4.4839 & 0.6256 & 0.00 & 154 & 181 & 34.0598 & 3 & 13 & 7.3084 & 14 & 63 & 39.7601 \\
\hline B74 & 3 & 3 & 3.3871 & 0.5584 & 0.00 & 154 & 163 & 4.2860 & 3 & 12 & 6.5822 & 18 & 53 & 27.1781 \\
\hline B75 & 11 & 11 & 11.6129 & 0.9892 & 0.00 & 168 & 183 & 13.2764 & 4 & 18 & 9.8581 & 18 & 89 & 45.1418 \\
\hline B76 & 5 & 5 & 5.1935 & 0.6011 & 0.00 & 163 & 193 & 35.1540 & 4 & 13 & 6.0851 & 20 & 70 & 40.9876 \\
\hline B77 & 5 & 5 & 5.2581 & 0.6816 & 0.00 & 154 & 186 & 23.2987 & 4 & 15 & 8.8359 & 24 & 73 & 39.9299 \\
\hline B78 & 4 & 4 & 4.7097 & 1.1887 & 0.00 & 156 & 170 & 10.0336 & 5 & 15 & 6.8506 & 23 & 69 & 31.6560 \\
\hline B79 & 14 & 14 & 14.0000 & 0.0000 & 0.00 & 156 & 164 & 6.0066 & 3 & 10 & 3.5484 & 12 & 46 & 15.1387 \\
\hline B80 & 11 & 11 & 11.0000 & 0.0000 & 0.00 & 153 & 158 & 6.2756 & 5 & 11 & 3.4811 & 20 & 48 & 14.9292 \\
\hline B81 & 11 & 11 & 11.4839 & 0.9263 & 0.00 & 154 & 159 & 5.5222 & 3 & 12 & 7.9278 & 16 & 54 & 31.9301 \\
\hline B82 & 10 & 10 & 10.5484 & 0.8500 & 0.00 & 154 & 156 & 2.3344 & 4 & 12 & 5.7675 & 16 & 51 & 24.5353 \\
\hline B83 & 12 & 12 & 12.0000 & 0.0000 & 0.00 & 157 & 164 & 4.1408 & 4 & 13 & 6.6827 & 16 & 60 & 29.7937 \\
\hline B84 & 12 & 12 & 12.0645 & 0.3592 & 0.00 & 154 & 158 & 5.3961 & 4 & 11 & 5.1974 & 16 & 49 & 22.7398 \\
\hline B85 & 8 & 8 & 8.9032 & 1.4687 & 0.00 & 154 & 157 & 2.9860 & 5 & 11 & 4.7201 & 21 & 47 & 19.4751 \\
\hline B86 & 8 & 8 & 8.2258 & 0.8835 & 0.00 & 154 & 156 & 2.0765 & 3 & 8 & 4.5371 & 12 & 34 & 18.4101 \\
\hline B87 & 10 & 10 & 10.5161 & 0.9957 & 0.00 & 162 & 171 & 4.9756 & 7 & 19 & 8.8350 & 29 & 87 & 40.9288 \\
\hline B88 & 8 & 8 & 8.0000 & 0.0000 & 0.00 & 154 & 157 & 2.9647 & 4 & 10 & 4.8012 & 17 & 43 & 20.1409 \\
\hline B89 & 8 & 8 & 8.0000 & 0.0000 & 0.00 & 154 & 157 & 3.8529 & 4 & 10 & 4.7071 & 18 & 45 & 19.3560 \\
\hline \multirow[t]{2}{*}{ B90 } & 5 & 5 & 5.1935 & 0.7492 & 0.00 & 154 & 156 & 1.4398 & 3 & 10 & 4.2256 & 12 & 43 & 17.4126 \\
\hline & & AVG & 8.1079 & 0.4472 & 0.00 & 169 & 195 & 26.3893 & 2 & 9 & 4.8796 & 13 & 49 & 27.5041 \\
\hline
\end{tabular}


TABLE 32: MBO using parallel quick sort algorithm with 16 threads.

\begin{tabular}{|c|c|c|c|c|c|c|c|c|c|c|c|c|c|c|}
\hline \multirow{2}{*}{ ID } & \multirow{2}{*}{ OPT } & \multicolumn{4}{|c|}{ Optimum } & \multicolumn{3}{|c|}{ Execution time } & \multicolumn{3}{|c|}{ Cycle } & \multicolumn{3}{|c|}{ Time cycle } \\
\hline & & $\mathrm{MBO}$ & AVG & $\mathrm{SD}$ & RPD\% & BET & AVG & ST & $\mathrm{BC}$ & AVG & $\mathrm{SD}$ & BTC & AVG & SD \\
\hline B1 & 11 & 11 & 11.0000 & 0.0000 & 0.00 & 157 & 177 & 45.0254 & 2 & 6 & 3.3999 & 12 & 34 & 19.8172 \\
\hline B2 & 11 & 11 & 11.0000 & 0.0000 & 0.00 & 152 & 157 & 6.9088 & 2 & 3 & 1.0555 & 8 & 16 & 5.8123 \\
\hline B3 & 11 & 11 & 11.0000 & 0.0000 & 0.00 & 152 & 157 & 6.2524 & 2 & 4 & 1.4760 & 8 & 20 & 7.8748 \\
\hline B4 & 11 & 11 & 11.0000 & 0.0000 & 0.00 & 154 & 178 & 34.0738 & 2 & 4 & 1.8198 & 9 & 22 & 13.7301 \\
\hline B5 & 11 & 11 & 11.0000 & 0.0000 & 0.00 & 155 & 176 & 26.4524 & 1 & 5 & 2.4065 & 4 & 25 & 11.7152 \\
\hline B6 & 7 & 7 & 7.0000 & 0.0000 & 0.00 & 155 & 197 & 56.6853 & 2 & 9 & 5.5449 & 10 & 47 & 28.6512 \\
\hline B7 & 6 & 6 & 6.0000 & 0.0000 & 0.00 & 152 & 203 & 48.1049 & 3 & 7 & 3.5948 & 16 & 41 & 32.0212 \\
\hline B8 & 4 & 4 & 4.1935 & 0.6011 & 0.00 & 157 & 173 & 22.7143 & 2 & 9 & 7.1292 & 8 & 42 & 31.6330 \\
\hline B9 & 3 & 3 & 3.0645 & 0.2497 & 0.00 & 154 & 166 & 16.3615 & 2 & 6 & 5.1249 & 9 & 27 & 21.8324 \\
\hline $\mathrm{B} 10$ & 3 & 3 & 3.0000 & 0.0000 & 0.00 & 156 & 184 & 33.1923 & 2 & 4 & 1.4323 & 8 & 24 & 19.4711 \\
\hline B11 & 4 & 4 & 4.2258 & 0.4250 & 0.00 & 163 & 232 & 56.6515 & 4 & 10 & 5.7893 & 22 & 62 & 40.3968 \\
\hline B12 & 4 & 4 & 4.0000 & 0.0000 & 0.00 & 157 & 187 & 39.6154 & 2 & 5 & 1.9962 & 8 & 27 & 14.4440 \\
\hline B13 & 4 & 4 & 4.0000 & 0.0000 & 0.00 & 154 & 168 & 11.7917 & 1 & 6 & 3.1890 & 4 & 28 & 13.8900 \\
\hline B14 & 3 & 3 & 3.4516 & 0.5059 & 0.00 & 153 & 196 & 78.8003 & 2 & 5 & 2.7549 & 8 & 31 & 37.8248 \\
\hline B15 & 1 & 1 & 1.5806 & 1.0255 & 0.00 & 152 & 175 & 23.7062 & 2 & 5 & 2.9523 & 8 & 29 & 17.7501 \\
\hline B16 & 14 & 14 & 14.0000 & 0.0000 & 0.00 & 154 & 191 & 21.0426 & 3 & 8 & 3.8362 & 12 & 43 & 22.4885 \\
\hline B17 & 13 & 13 & 13.0000 & 0.0000 & 0.00 & 155 & 177 & 30.7922 & 2 & 4 & 1.3190 & 8 & 22 & 5.8902 \\
\hline B18 & 13 & 13 & 13.0000 & 0.0000 & 0.00 & 154 & 167 & 11.8313 & 2 & 5 & 2.6011 & 10 & 26 & 10.8408 \\
\hline B19 & 13 & 13 & 13.0968 & 0.5388 & 0.00 & 154 & 170 & 17.4841 & 3 & 8 & 4.9782 & 12 & 36 & 23.2831 \\
\hline B20 & 13 & 13 & 13.0000 & 0.0000 & 0.00 & 153 & 166 & 11.7813 & 1 & 6 & 4.9294 & 7 & 31 & 23.0983 \\
\hline B21 & 9 & 9 & 9.0968 & 0.3005 & 0.00 & 157 & 183 & 16.9218 & 1 & 9 & 5.6618 & 5 & 46 & 26.0856 \\
\hline B22 & 6 & 6 & 6.0968 & 0.3005 & 0.00 & 154 & 177 & 24.5003 & 2 & 5 & 2.2590 & 8 & 25 & 13.5459 \\
\hline B23 & 6 & 6 & 6.2258 & 0.4250 & 0.00 & 154 & 165 & 14.6940 & 3 & 8 & 6.6832 & 13 & 36 & 30.1027 \\
\hline B24 & 5 & 5 & 5.2903 & 0.5884 & 0.00 & 154 & 168 & 13.6450 & 3 & 6 & 4.7176 & 12 & 29 & 19.0212 \\
\hline B25 & 4 & 4 & 4.3226 & 0.6525 & 0.00 & 152 & 173 & 25.1826 & 1 & 5 & 2.3233 & 4 & 24 & 11.7187 \\
\hline B26 & 5 & 5 & 5.0000 & 0.0000 & 0.00 & 155 & 177 & 24.4236 & 2 & 7 & 4.0783 & 8 & 32 & 18.5792 \\
\hline B27 & 3 & 3 & 3.0968 & 0.3005 & 0.00 & 153 & 165 & 10.0336 & 1 & 6 & 2.6303 & 4 & 28 & 11.0112 \\
\hline B28 & 3 & 3 & 3.1613 & 0.4544 & 0.00 & 154 & 175 & 22.3457 & 1 & 6 & 3.1084 & 4 & 27 & 13.0248 \\
\hline B29 & 3 & 3 & 3.0323 & 0.1796 & 0.00 & 156 & 166 & 14.2496 & 3 & 5 & 2.0457 & 12 & 23 & 9.0459 \\
\hline B30 & 2 & 2 & 2.4839 & 0.6256 & 0.00 & 153 & 162 & 10.4239 & 2 & 8 & 7.0603 & 8 & 34 & 28.7763 \\
\hline B31 & 7 & 7 & 7.0000 & 0.0000 & 0.00 & 163 & 175 & 10.1719 & 2 & 8 & 4.4158 & 11 & 37 & 20.2809 \\
\hline B32 & 4 & 4 & 4.0968 & 0.5388 & 0.00 & 154 & 171 & 16.5987 & 2 & 6 & 3.3376 & 8 & 28 & 16.8551 \\
\hline B33 & 4 & 4 & 4.2581 & 0.6816 & 0.00 & 154 & 165 & 13.2649 & 2 & 5 & 3.3237 & 8 & 22 & 13.7882 \\
\hline B34 & 4 & 4 & 4.1290 & 0.4995 & 0.00 & 154 & 175 & 30.9978 & 1 & 5 & 4.1265 & 4 & 27 & 22.0238 \\
\hline B35 & 4 & 4 & 4.5806 & 0.9228 & 0.00 & 153 & 178 & 96.2795 & 2 & 5 & 2.2337 & 8 & 23 & 9.5409 \\
\hline B36 & 13 & 13 & 13.0000 & 0.0000 & 0.00 & 154 & 167 & 13.6572 & 2 & 9 & 4.7026 & 8 & 40 & 19.6464 \\
\hline B37 & 10 & 10 & 10.6452 & 0.9504 & 0.00 & 152 & 156 & 2.9487 & 1 & 5 & 3.7108 & 4 & 23 & 15.0990 \\
\hline B38 & 8 & 8 & 8.0645 & 0.2497 & 0.00 & 152 & 155 & 2.0182 & 3 & 11 & 8.6129 & 12 & 45 & 34.9003 \\
\hline B39 & 5 & 5 & 5.0000 & 0.0000 & 0.00 & 152 & 156 & 2.2874 & 2 & 4 & 2.1677 & 8 & 20 & 9.0137 \\
\hline B40 & 5 & 5 & 5.1290 & 0.4995 & 0.00 & 152 & 155 & 2.8021 & 3 & 6 & 4.3584 & 12 & 25 & 17.7059 \\
\hline B41 & 8 & 8 & 8.0000 & 0.0000 & 0.00 & 155 & 160 & 4.9095 & 3 & 9 & 5.2548 & 12 & 39 & 21.6216 \\
\hline B42 & 8 & 8 & 8.0000 & 0.0000 & 0.00 & 153 & 157 & 4.8928 & 2 & 4 & 2.8744 & 8 & 20 & 12.4631 \\
\hline B43 & 8 & 8 & 8.0000 & 0.0000 & 0.00 & 152 & 163 & 9.2213 & 1 & 5 & 3.8633 & 4 & 24 & 16.0156 \\
\hline B44 & 5 & 5 & 6.4516 & 1.5240 & 0.00 & 154 & 171 & 24.9946 & 2 & 5 & 2.4132 & 8 & 23 & 14.3787 \\
\hline B45 & 5 & 5 & 5.9677 & 1.4256 & 0.00 & 154 & 168 & 16.9289 & 2 & 6 & 2.4323 & 8 & 26 & 10.5386 \\
\hline B46 & 8 & 8 & 8.0323 & 0.1796 & 0.00 & 160 & 192 & 33.4808 & 2 & 10 & 8.0861 & 10 & 56 & 44.0563 \\
\hline B47 & 5 & 5 & 5.1935 & 0.7492 & 0.00 & 155 & 176 & 28.5692 & 2 & 5 & 2.5539 & 8 & 27 & 19.4449 \\
\hline B48 & 5 & 5 & 5.1935 & 0.6011 & 0.00 & 153 & 166 & 16.1625 & 2 & 5 & 2.4569 & 8 & 25 & 12.9483 \\
\hline B49 & 5 & 5 & 5.2581 & 1.1245 & 0.00 & 154 & 185 & 24.4041 & 2 & 6 & 4.2487 & 12 & 34 & 26.5968 \\
\hline B50 & 5 & 5 & 5.2581 & 0.6816 & 0.00 & 152 & 163 & 12.7533 & 2 & 5 & 2.7309 & 8 & 24 & 12.5672 \\
\hline
\end{tabular}


TABle 32: Continued.

\begin{tabular}{|c|c|c|c|c|c|c|c|c|c|c|c|c|c|c|}
\hline \multirow{2}{*}{ ID } & \multirow{2}{*}{ OPT } & \multicolumn{4}{|c|}{ Optimum } & \multicolumn{3}{|c|}{ Execution time } & \multicolumn{3}{|c|}{ Cycle } & \multicolumn{3}{|c|}{ Time cycle } \\
\hline & & $\mathrm{MBO}$ & AVG & SD & $\mathrm{RPD} \%$ & $\mathrm{BET}$ & AVG & ST & $\mathrm{BC}$ & AVG & SD & BTC & AVG & SD \\
\hline B51 & 27 & 27 & 28.1290 & 1.1472 & 0.00 & 155 & 163 & 4.5195 & 2 & 18 & 8.4909 & 9 & 81 & 36.9657 \\
\hline B52 & 18 & 18 & 18.9032 & 0.9436 & 0.00 & 155 & 159 & 3.0589 & 5 & 20 & 9.0487 & 22 & 86 & 37.0107 \\
\hline B53 & 11 & 11 & 11.0000 & 0.0000 & 0.00 & 154 & 158 & 3.9611 & 4 & 10 & 3.8532 & 17 & 42 & 15.9685 \\
\hline B54 & 11 & 11 & 11.0000 & 0.0000 & 0.00 & 152 & 156 & 2.7739 & 3 & 8 & 2.9461 & 12 & 36 & 12.0974 \\
\hline B55 & 7 & 7 & 7.0000 & 0.0000 & 0.00 & 154 & 159 & 4.2006 & 4 & 9 & 3.7540 & 16 & 40 & 15.8327 \\
\hline B56 & 6 & 6 & 6.0000 & 0.0000 & 0.00 & 154 & 159 & 4.3319 & 2 & 11 & 4.5788 & 8 & 46 & 19.1962 \\
\hline B57 & 6 & 6 & 6.0645 & 0.2497 & 0.00 & 153 & 159 & 9.3087 & 5 & 12 & 5.6773 & 20 & 51 & 24.6464 \\
\hline B58 & 6 & 6 & 6.0645 & 0.2497 & 0.00 & 153 & 157 & 3.1748 & 5 & 10 & 5.7940 & 20 & 44 & 23.7433 \\
\hline B59 & 9 & 9 & 9.0645 & 0.2497 & 0.00 & 155 & 160 & 3.7944 & 3 & 13 & 6.6326 & 14 & 58 & 27.4709 \\
\hline B60 & 4 & 4 & 4.6452 & 1.7233 & 0.00 & 154 & 158 & 5.7524 & 6 & 14 & 5.8481 & 25 & 58 & 25.0316 \\
\hline B61 & 4 & 4 & 4.0645 & 0.2497 & 0.00 & 153 & 157 & 3.0000 & 3 & 11 & 4.8558 & 12 & 48 & 20.1127 \\
\hline B62 & 4 & 4 & 4.0000 & 0.0000 & 0.00 & 152 & 155 & 3.5305 & 2 & 10 & 5.8902 & 8 & 44 & 25.2071 \\
\hline B63 & 27 & 27 & 27.2581 & 0.4448 & 0.00 & 157 & 162 & 3.2847 & 4 & 17 & 10.0560 & 19 & 75 & 43.2526 \\
\hline B64 & 18 & 18 & 18.4516 & 0.9946 & 0.00 & 154 & 157 & 4.0473 & 4 & 20 & 8.9582 & 16 & 86 & 36.8209 \\
\hline B65 & 14 & 14 & 14.1290 & 0.3408 & 0.00 & 154 & 156 & 2.2562 & 5 & 18 & 8.3264 & 21 & 75 & 34.2576 \\
\hline B66 & 13 & 13 & 13.0000 & 0.0000 & 0.00 & 154 & 156 & 2.8368 & 5 & 10 & 3.5281 & 23 & 45 & 14.0460 \\
\hline B67 & 11 & 11 & 11.0968 & 0.3005 & 0.00 & 155 & 159 & 2.1357 & 3 & 11 & 5.8249 & 13 & 50 & 24.4252 \\
\hline B68 & 8 & 8 & 8.1290 & 0.3408 & 0.00 & 154 & 158 & 4.0166 & 3 & 13 & 6.0748 & 13 & 56 & 25.8166 \\
\hline B69 & 8 & 8 & 8.3226 & 0.5408 & 0.00 & 154 & 157 & 3.5042 & 3 & 10 & 3.6715 & 13 & 44 & 15.2921 \\
\hline B70 & 6 & 6 & 6.6129 & 0.6672 & 0.00 & 153 & 156 & 2.5003 & 3 & 12 & 5.7179 & 12 & 52 & 23.5929 \\
\hline B71 & 6 & 6 & 6.1290 & 0.4995 & 0.00 & 154 & 158 & 3.0494 & 4 & 12 & 6.1740 & 16 & 53 & 25.9316 \\
\hline B72 & 4 & 4 & 4.2258 & 0.4973 & 0.00 & 153 & 158 & 4.4504 & 4 & 13 & 7.0095 & 16 & 54 & 29.2744 \\
\hline B73 & 4 & 4 & 4.4839 & 0.7244 & 0.00 & 154 & 157 & 3.1088 & 5 & 15 & 8.0446 & 20 & 62 & 33.2164 \\
\hline B74 & 3 & 3 & 3.6129 & 0.6672 & 0.00 & 153 & 156 & 2.3601 & 4 & 14 & 7.6069 & 16 & 59 & 31.1370 \\
\hline B75 & 11 & 11 & 11.6452 & 0.9848 & 0.00 & 155 & 162 & 8.1721 & 3 & 16 & 9.6780 & 16 & 71 & 41.5330 \\
\hline B76 & 5 & 5 & 5.0645 & 0.3592 & 0.00 & 154 & 168 & 15.3240 & 5 & 12 & 4.8560 & 20 & 56 & 23.5850 \\
\hline B77 & 5 & 5 & 5.5806 & 0.9228 & 0.00 & 154 & 171 & 25.1381 & 3 & 15 & 9.7619 & 12 & 66 & 41.9013 \\
\hline B78 & 4 & 4 & 4.8065 & 1.2495 & 0.00 & 159 & 182 & 29.0268 & 4 & 12 & 6.2212 & 17 & 60 & 27.7959 \\
\hline B79 & 14 & 14 & 14.0000 & 0.0000 & 0.00 & 157 & 169 & 15.0903 & 3 & 9 & 3.8852 & 13 & 44 & 15.6725 \\
\hline B80 & 11 & 11 & 11.0000 & 0.0000 & 0.00 & 154 & 162 & 10.4361 & 4 & 11 & 3.9841 & 23 & 47 & 16.2254 \\
\hline B81 & 11 & 11 & 11.3548 & 0.8386 & 0.00 & 154 & 172 & 19.7290 & 7 & 15 & 7.2530 & 33 & 71 & 32.6199 \\
\hline B82 & 10 & 10 & 10.4839 & 0.7690 & 0.00 & 156 & 199 & 119.2439 & 5 & 12 & 6.1247 & 20 & 80 & 132.6427 \\
\hline B83 & 12 & 12 & 12.0000 & 0.0000 & 0.00 & 157 & 188 & 28.7156 & 3 & 11 & 5.8672 & 13 & 56 & 32.9586 \\
\hline B84 & 12 & 12 & 12.1935 & 0.6011 & 0.00 & 155 & 180 & 30.9175 & 6 & 11 & 5.1700 & 25 & 54 & 26.6157 \\
\hline B85 & 8 & 8 & 9.0645 & 1.4127 & 0.00 & 154 & 162 & 8.6101 & 3 & 12 & 5.6041 & 12 & 54 & 23.3782 \\
\hline B86 & 8 & 8 & 8.1290 & 0.7184 & 0.00 & 155 & 178 & 29.0973 & 2 & 10 & 5.2824 & 9 & 47 & 28.5301 \\
\hline B87 & 10 & 10 & 10.6452 & 1.0503 & 0.00 & 157 & 180 & 28.1470 & 4 & 15 & 6.8785 & 16 & 72 & 29.6574 \\
\hline B88 & 8 & 8 & 8.0000 & 0.0000 & 0.00 & 153 & 194 & 35.9039 & 2 & 9 & 3.5636 & 8 & 51 & 20.8887 \\
\hline B89 & 8 & 8 & 8.0323 & 0.1796 & 0.00 & 155 & 183 & 37.7474 & 3 & 10 & 4.7424 & 14 & 52 & 34.4475 \\
\hline \multirow[t]{2}{*}{ B90 } & 5 & 5 & 5.5806 & 1.2048 & 0.00 & 152 & 178 & 32.5974 & 4 & 10 & 5.5323 & 22 & 51 & 24.4181 \\
\hline & & AVG & 8.1072 & 0.4325 & 0.00 & 154 & 169 & 18.9543 & 2 & 8 & 4.7619 & 12 & 42 & 23.6231 \\
\hline
\end{tabular}


TABLE 33: MBO using parallel quick sort algorithm with 32 threads.

\begin{tabular}{|c|c|c|c|c|c|c|c|c|c|c|c|c|c|c|}
\hline \multirow{2}{*}{ ID } & \multirow{2}{*}{ OPT } & \multicolumn{4}{|c|}{ Optimum } & \multicolumn{3}{|c|}{ Execution time } & \multicolumn{3}{|c|}{ Cycle } & \multicolumn{3}{|c|}{ Time cycle } \\
\hline & & $\mathrm{MBO}$ & AVG & SD & RPD\% & BET & AVG & ST & $\mathrm{BC}$ & AVG & SD & BTC & AVG & SD \\
\hline B1 & 11 & 11 & 11.0000 & 0.0000 & 0.00 & 157 & 193 & 67.2126 & 4 & 8 & 3.8529 & 19 & 47 & 23.5622 \\
\hline B2 & 11 & 11 & 11.0000 & 0.0000 & 0.00 & 153 & 160 & 6.2348 & 2 & 4 & 1.3190 & 9 & 19 & 7.0225 \\
\hline B3 & 11 & 11 & 11.0000 & 0.0000 & 0.00 & 153 & 158 & 4.9071 & 1 & 4 & 1.8020 & 4 & 19 & 7.5696 \\
\hline B4 & 11 & 11 & 11.0000 & 0.0000 & 0.00 & 152 & 156 & 2.5745 & 2 & 4 & 1.6428 & 8 & 16 & 6.8760 \\
\hline B5 & 11 & 11 & 11.0000 & 0.0000 & 0.00 & 154 & 160 & 5.0292 & 2 & 6 & 3.4962 & 8 & 29 & 14.8304 \\
\hline B6 & 7 & 7 & 7.0000 & 0.0000 & 0.00 & 160 & 195 & 70.3551 & 3 & 11 & 7.8781 & 12 & 60 & 43.3428 \\
\hline B7 & 6 & 6 & 6.0000 & 0.0000 & 0.00 & 155 & 160 & 3.6423 & 2 & 5 & 2.2538 & 9 & 24 & 9.5139 \\
\hline B8 & 4 & 4 & 4.1935 & 0.6011 & 0.00 & 154 & 160 & 4.4473 & 2 & 13 & 10.0692 & 8 & 56 & 41.9610 \\
\hline B9 & 3 & 3 & 3.0323 & 0.1796 & 0.00 & 153 & 158 & 3.1302 & 2 & 5 & 2.6901 & 8 & 22 & 11.0625 \\
\hline $\mathrm{B} 10$ & 3 & 3 & 3.0000 & 0.0000 & 0.00 & 153 & 161 & 5.0956 & 2 & 4 & 1.9092 & 8 & 19 & 8.2968 \\
\hline B11 & 4 & 4 & 4.1613 & 0.3739 & 0.00 & 159 & 174 & 8.7291 & 2 & 11 & 9.6157 & 10 & 55 & 44.6467 \\
\hline B12 & 4 & 4 & 4.0000 & 0.0000 & 0.00 & 154 & 158 & 3.0515 & 2 & 4 & 2.0483 & 8 & 20 & 8.5759 \\
\hline B13 & 4 & 4 & 4.0645 & 0.3592 & 0.00 & 153 & 157 & 3.8356 & 2 & 4 & 1.7677 & 8 & 18 & 7.3472 \\
\hline B14 & 3 & 3 & 3.4516 & 0.5059 & 0.00 & 152 & 156 & 3.0789 & 2 & 5 & 3.0164 & 9 & 24 & 12.6226 \\
\hline B15 & 1 & 1 & 1.2581 & 0.6816 & 0.00 & 152 & 158 & 7.3659 & 2 & 6 & 2.6069 & 8 & 25 & 11.2249 \\
\hline B16 & 14 & 14 & 14.0000 & 0.0000 & 0.00 & 154 & 168 & 8.2101 & 2 & 7 & 3.0670 & 10 & 35 & 13.2306 \\
\hline B17 & 13 & 13 & 13.0000 & 0.0000 & 0.00 & 154 & 159 & 3.4867 & 2 & 5 & 1.9389 & 8 & 22 & 8.2489 \\
\hline B18 & 13 & 13 & 13.0000 & 0.0000 & 0.00 & 152 & 156 & 2.6437 & 2 & 4 & 2.0288 & 8 & 20 & 8.4173 \\
\hline B19 & 13 & 13 & 13.0000 & 0.0000 & 0.00 & 152 & 159 & 6.3924 & 2 & 6 & 2.6567 & 8 & 27 & 11.0241 \\
\hline B20 & 13 & 13 & 13.0000 & 0.0000 & 0.00 & 152 & 158 & 8.2973 & 2 & 7 & 3.5360 & 8 & 30 & 14.4425 \\
\hline B21 & 9 & 9 & 9.1290 & 0.3408 & 0.00 & 163 & 180 & 10.9634 & 2 & 9 & 6.9766 & 9 & 47 & 33.4055 \\
\hline B22 & 6 & 6 & 6.0645 & 0.2497 & 0.00 & 153 & 159 & 3.6133 & 1 & 6 & 3.4591 & 4 & 29 & 14.6736 \\
\hline B23 & 6 & 6 & 6.1935 & 0.4016 & 0.00 & 154 & 159 & 5.3000 & 2 & 7 & 5.8411 & 12 & 32 & 24.2679 \\
\hline B24 & 5 & 5 & 5.4516 & 0.6752 & 0.00 & 152 & 157 & 3.6320 & 2 & 6 & 2.7658 & 8 & 25 & 11.7582 \\
\hline B25 & 4 & 4 & 4.2258 & 0.4250 & 0.00 & 152 & 157 & 3.4273 & 2 & 7 & 4.7573 & 8 & 29 & 19.6816 \\
\hline B26 & 5 & 5 & 5.0323 & 0.1796 & 0.00 & 155 & 167 & 7.9712 & 1 & 8 & 5.1750 & 4 & 38 & 22.6477 \\
\hline B27 & 3 & 3 & 3.0645 & 0.2497 & 0.00 & 154 & 160 & 5.4513 & 2 & 6 & 4.0284 & 8 & 28 & 17.1102 \\
\hline B28 & 3 & 3 & 3.1290 & 0.3408 & 0.00 & 152 & 157 & 3.9081 & 3 & 5 & 1.7096 & 12 & 23 & 7.4421 \\
\hline B29 & 3 & 3 & 3.0645 & 0.2497 & 0.00 & 152 & 157 & 4.1860 & 1 & 5 & 2.5911 & 4 & 21 & 10.8956 \\
\hline B30 & 2 & 2 & 2.5806 & 0.5642 & 0.00 & 152 & 157 & 3.6694 & 2 & 7 & 6.6775 & 8 & 31 & 28.3555 \\
\hline B31 & 7 & 7 & 7.0000 & 0.0000 & 0.00 & 155 & 165 & 6.5364 & 1 & 8 & 4.6596 & 4 & 37 & 21.1130 \\
\hline B32 & 4 & 4 & 4.1935 & 0.7492 & 0.00 & 153 & 159 & 4.2621 & 1 & 8 & 5.6591 & 4 & 34 & 23.4602 \\
\hline B33 & 4 & 4 & 4.1290 & 0.4995 & 0.00 & 152 & 157 & 3.4093 & 3 & 6 & 3.2015 & 12 & 25 & 13.2534 \\
\hline B34 & 4 & 4 & 4.3226 & 0.7478 & 0.00 & 153 & 157 & 3.5469 & 2 & 5 & 4.2812 & 9 & 24 & 17.1984 \\
\hline B35 & 4 & 4 & 4.3871 & 0.8032 & 0.00 & 153 & 159 & 6.8737 & 1 & 5 & 2.7591 & 4 & 24 & 11.7173 \\
\hline B36 & 13 & 13 & 13.0000 & 0.0000 & 0.00 & 162 & 191 & 55.9882 & 1 & 8 & 5.3864 & 4 & 42 & 27.3086 \\
\hline B37 & 10 & 10 & 10.3226 & 0.7478 & 0.00 & 154 & 163 & 7.1865 & 1 & 8 & 7.0555 & 5 & 35 & 30.6683 \\
\hline B38 & 8 & 8 & 8.0000 & 0.0000 & 0.00 & 152 & 158 & 3.9570 & 3 & 9 & 6.1737 & 12 & 38 & 25.0810 \\
\hline B39 & 5 & 5 & 5.0000 & 0.0000 & 0.00 & 154 & 159 & 4.1968 & 2 & 5 & 2.3047 & 8 & 22 & 10.0090 \\
\hline B40 & 5 & 5 & 5.1935 & 0.6011 & 0.00 & 154 & 162 & 8.1181 & 3 & 5 & 1.7506 & 12 & 22 & 7.9040 \\
\hline B41 & 8 & 8 & 8.3226 & 1.2487 & 0.00 & 156 & 172 & 11.4272 & 3 & 11 & 7.4741 & 14 & 55 & 38.2555 \\
\hline B42 & 8 & 8 & 8.0000 & 0.0000 & 0.00 & 155 & 163 & 9.3587 & 2 & 4 & 2.2855 & 8 & 21 & 10.4073 \\
\hline B43 & 8 & 8 & 8.0000 & 0.0000 & 0.00 & 155 & 215 & 74.3419 & 2 & 5 & 2.0483 & 9 & 29 & 16.7208 \\
\hline B44 & 5 & 5 & 5.8710 & 1.3842 & 0.00 & 155 & 208 & 74.1396 & 2 & 6 & 3.7788 & 12 & 35 & 20.5935 \\
\hline B45 & 5 & 5 & 6.3548 & 1.5176 & 0.00 & 152 & 174 & 54.0282 & 1 & 5 & 2.9910 & 12 & 26 & 14.9598 \\
\hline B46 & 8 & 8 & 8.0323 & 0.1796 & 0.00 & 159 & 266 & 116.1782 & 1 & 10 & 5.7489 & 5 & 65 & 52.7578 \\
\hline B47 & 5 & 5 & 5.0968 & 0.5388 & 0.00 & 166 & 197 & 82.7696 & 1 & 5 & 2.1287 & 7 & 25 & 11.5699 \\
\hline B48 & 5 & 5 & 5.2581 & 0.6816 & 0.00 & 164 & 241 & 91.5422 & 2 & 5 & 2.6392 & 8 & 40 & 34.3044 \\
\hline B49 & 5 & 5 & 5.1935 & 0.6011 & 0.00 & 169 & 281 & 103.5425 & 2 & 7 & 4.7853 & 10 & 49 & 38.4886 \\
\hline B50 & 5 & 5 & 5.3226 & 0.7478 & 0.00 & 157 & 196 & 37.8474 & 2 & 5 & 3.0547 & 9 & 29 & 15.1232 \\
\hline
\end{tabular}


TABle 33: Continued.

\begin{tabular}{|c|c|c|c|c|c|c|c|c|c|c|c|c|c|c|}
\hline \multirow{2}{*}{ ID } & \multirow{2}{*}{ OPT } & \multicolumn{4}{|c|}{ Optimum } & \multicolumn{3}{|c|}{ Execution time } & \multicolumn{3}{|c|}{ Cycle } & \multicolumn{3}{|c|}{ Time cycle } \\
\hline & & $\mathrm{MBO}$ & AVG & SD & RPD\% & BET & AVG & ST & $\mathrm{BC}$ & AVG & SD & BTC & AVG & $\mathrm{SD}$ \\
\hline B51 & 27 & 27 & 28.0645 & 0.8920 & 0.00 & 158 & 207 & 50.7988 & 3 & 20 & 9.7651 & 15 & 115 & 63.2991 \\
\hline B52 & 18 & 18 & 18.7742 & 1.0555 & 0.00 & 160 & 232 & 64.3241 & 4 & 20 & 9.6569 & 17 & 124 & 71.2839 \\
\hline B53 & 11 & 11 & 11.0000 & 0.0000 & 0.00 & 161 & 174 & 14.6625 & 6 & 11 & 4.6327 & 26 & 50 & 22.0361 \\
\hline B54 & 11 & 11 & 11.0000 & 0.0000 & 0.00 & 158 & 193 & 115.3675 & 4 & 8 & 2.3550 & 18 & 40 & 12.4287 \\
\hline B55 & 7 & 7 & 7.1290 & 0.7184 & 0.00 & 169 & 180 & 6.2545 & 4 & 12 & 5.4335 & 21 & 59 & 26.9609 \\
\hline B56 & 6 & 6 & 6.0000 & 0.0000 & 0.00 & 163 & 174 & 13.8653 & 4 & 9 & 2.4705 & 18 & 42 & 12.1870 \\
\hline B57 & 6 & 6 & 6.2258 & 0.6688 & 0.00 & 161 & 178 & 20.6817 & 5 & 11 & 6.1501 & 26 & 55 & 26.7029 \\
\hline B58 & 6 & 6 & 6.0323 & 0.1796 & 0.00 & 161 & 166 & 5.6791 & 2 & 9 & 5.4692 & 10 & 40 & 22.9230 \\
\hline B59 & 9 & 9 & 9.0968 & 0.3005 & 0.00 & 170 & 178 & 4.8449 & 4 & 12 & 5.8635 & 19 & 60 & 27.2347 \\
\hline B60 & 4 & 4 & 4.2581 & 0.9989 & 0.00 & 162 & 172 & 7.4626 & 3 & 11 & 6.1011 & 14 & 54 & 28.2474 \\
\hline B61 & 4 & 4 & 4.0645 & 0.2497 & 0.00 & 159 & 167 & 5.3539 & 4 & 11 & 5.2357 & 18 & 49 & 23.0532 \\
\hline B62 & 4 & 4 & 4.0968 & 0.3005 & 0.00 & 159 & 174 & 11.7428 & 2 & 11 & 5.9301 & 9 & 51 & 28.0555 \\
\hline B63 & 27 & 27 & 27.2258 & 0.4250 & 0.00 & 176 & 183 & 4.3125 & 5 & 18 & 10.3340 & 24 & 89 & 50.4055 \\
\hline B64 & 18 & 18 & 18.2581 & 0.6308 & 0.00 & 163 & 197 & 50.6838 & 4 & 16 & 9.1136 & 19 & 82 & 44.4143 \\
\hline B65 & 14 & 14 & 14.0000 & 0.0000 & 0.00 & 155 & 165 & 15.1038 & 6 & 16 & 8.3361 & 24 & 69 & 36.6212 \\
\hline B66 & 13 & 13 & 13.0000 & 0.0000 & 0.00 & 155 & 164 & 9.6089 & 4 & 9 & 3.0533 & 19 & 42 & 14.9758 \\
\hline B67 & 11 & 11 & 11.1290 & 0.3408 & 0.00 & 154 & 172 & 13.8726 & 3 & 13 & 8.6648 & 13 & 62 & 38.9473 \\
\hline B68 & 8 & 8 & 8.5484 & 1.4338 & 0.00 & 154 & 161 & 8.0448 & 2 & 13 & 6.7357 & 8 & 57 & 28.8067 \\
\hline B69 & 8 & 8 & 8.5806 & 0.7199 & 0.00 & 152 & 189 & 51.5676 & 5 & 11 & 4.3643 & 20 & 55 & 26.5377 \\
\hline B70 & 6 & 6 & 6.3871 & 0.6152 & 0.00 & 153 & 177 & 47.0718 & 6 & 13 & 5.1690 & 24 & 66 & 39.7750 \\
\hline B71 & 6 & 6 & 6.0000 & 0.0000 & 0.00 & 161 & 218 & 88.2505 & 7 & 12 & 4.1688 & 34 & 75 & 43.2647 \\
\hline B72 & 4 & 4 & 4.1935 & 0.4774 & 0.00 & 154 & 171 & 22.0755 & 4 & 13 & 7.8702 & 17 & 64 & 42.7791 \\
\hline B73 & 4 & 4 & 4.6774 & 0.5408 & 0.00 & 156 & 207 & 66.9800 & 4 & 15 & 7.8781 & 16 & 90 & 80.4632 \\
\hline B74 & 3 & 3 & 3.4839 & 0.6768 & 0.00 & 158 & 225 & 117.9903 & 4 & 13 & 8.8637 & 18 & 69 & 43.8618 \\
\hline B75 & 11 & 11 & 11.6774 & 0.8713 & 0.00 & 175 & 251 & 58.8080 & 5 & 17 & 7.5933 & 34 & 110 & 56.0249 \\
\hline B76 & 5 & 5 & 5.1290 & 0.4995 & 0.00 & 155 & 179 & 27.7539 & 4 & 13 & 7.2367 & 22 & 63 & 29.9982 \\
\hline B77 & 5 & 5 & 5.5484 & 0.9605 & 0.00 & 152 & 159 & 5.4345 & 5 & 12 & 7.0432 & 20 & 51 & 28.9970 \\
\hline B78 & 4 & 4 & 4.6452 & 1.1120 & 0.00 & 152 & 163 & 20.6672 & 3 & 11 & 6.2579 & 12 & 49 & 26.2351 \\
\hline B79 & 14 & 14 & 14.0000 & 0.0000 & 0.00 & 154 & 165 & 8.7211 & 3 & 9 & 3.3344 & 15 & 42 & 15.1123 \\
\hline B80 & 11 & 11 & 11.0000 & 0.0000 & 0.00 & 152 & 214 & 74.0166 & 6 & 10 & 3.1398 & 26 & 58 & 30.2040 \\
\hline B81 & 11 & 11 & 11.5484 & 1.0595 & 0.00 & 152 & 192 & 74.2480 & 5 & 16 & 9.2194 & 20 & 85 & 61.0593 \\
\hline B82 & 10 & 10 & 10.4839 & 0.7244 & 0.00 & 152 & 163 & 8.3600 & 5 & 11 & 5.7070 & 22 & 50 & 25.8347 \\
\hline B83 & 12 & 12 & 12.0000 & 0.0000 & 0.00 & 153 & 159 & 4.4325 & 4 & 13 & 7.7335 & 16 & 55 & 32.6401 \\
\hline B84 & 12 & 12 & 12.0645 & 0.3592 & 0.00 & 154 & 162 & 6.8866 & 4 & 11 & 5.2595 & 20 & 51 & 22.9838 \\
\hline B85 & 8 & 8 & 9.1290 & 1.4774 & 0.00 & 153 & 160 & 4.7076 & 4 & 13 & 7.8904 & 18 & 56 & 32.4863 \\
\hline B86 & 8 & 8 & 8.0645 & 0.2497 & 0.00 & 152 & 173 & 39.7751 & 6 & 11 & 3.4938 & 24 & 53 & 24.8335 \\
\hline B87 & 10 & 10 & 10.3871 & 0.8032 & 0.00 & 154 & 247 & 122.0646 & 6 & 18 & 8.3162 & 32 & 127 & 103.6875 \\
\hline B88 & 8 & 8 & 8.0000 & 0.0000 & 0.00 & 152 & 160 & 7.8272 & 4 & 9 & 3.9459 & 16 & 40 & 16.1653 \\
\hline B89 & 8 & 8 & 8.0000 & 0.0000 & 0.00 & 154 & 159 & 3.6638 & 6 & 10 & 3.9273 & 24 & 42 & 16.1224 \\
\hline \multirow[t]{2}{*}{ B90 } & 5 & 5 & 5.5806 & 1.2048 & 0.00 & 153 & 158 & 5.3959 & 4 & 10 & 3.8771 & 16 & 45 & 16.0706 \\
\hline & & AVG & 8.0953 & 0.4219 & 0.00 & 156 & 177 & 25.8717 & 3 & 9 & 4.8993 & 13 & 46 & 25.9852 \\
\hline
\end{tabular}




\section{Acknowledgments}

Boris Almonacid is supported by Postgraduate Grant Pontificia Universidad Católica de Valparaíso 2015 (INF-PUCV 2015). Ricardo Soto is supported by Grant CONICYT/FONDECYT/INICIACION/11130459. Broderick Crawford is supported by Grant CONICYT/FONDECYT/ REGULAR/1140897. Fernando Paredes is supported by Grant CONICYT/FONDECYT/1130455.

\section{References}

[1] C. Mosier and L. Taube, "The facets of group technology and their impacts on implementation - a state-of-the-art survey," Omega, vol. 13, no. 5, pp. 381-391, 1985.

[2] F. F. Boctor, "A Jinear formulation of the machine-part cell formation problem," International Journal of Production Research, vol. 29, no. 2, pp. 343-356, 1991.

[3] O. Durán, N. Rodriguez, and L. A. Consalter, "Collaborative particle swarm optimization with a data mining technique for manufacturing cell design," Expert Systems with Applications, vol. 37, no. 2, pp. 1563-1567, 2010.

[4] R. Soto, B. Crawford, E. Vega, and F. Paredes, "Solving manufacturing cell design problems using an artificial fish swarm algorithm," in Advances in Artificial Intelligence and Soft Computing, pp. 282-290, Springer, 2015.

[5] G. F. K. Purcheck, "A linear-programming method for the combinatorial grouping of an incomplete power set," Journal of Cybernetics, vol. 5, no. 4, pp. 51-76, 1975.

[6] S. M. Shafer and D. F. Rogers, "A goal programming approach to the cell formation problem," Journal of Operations Management, vol. 10, no. 1, pp. 28-43, 1991.

[7] J. A. Joines, M. G. Kay, R. E. King, and C. T. Culbreth, "A hybrid genetic algorithm for manufacturing cell design," Journal of the Chinese Institute of Industrial Engineers, vol. 17, no. 5, pp. 549564, 2000.

[8] R. Soto, H. Kjellerstrand, O. Durán, B. Crawford, E. Monfroy, and F. Paredes, "Cell formation in group technology using constraint programming and boolean satisfiability," Expert Systems with Applications, vol. 39, no. 13, pp. 11423-11427, 2012.

[9] R. Soto, H. Kjellerstrand, J. Gutiérrez, A. López, B. Crawford, and E. Monfroy, "Solving manufacturing cell design problems using constraint programming," in Advanced Research in Applied Artificial Intelligence, H. Jiang, W. Ding, M. Ali, and X. Wu, Eds., vol. 7345 of Lecture Notes in Computer Science, pp. 400-406, Springer, 2012.

[10] H. M. Selim, R. G. Askin, and A. J. Vakharia, "Cell formation in group technology: review, evaluation and directions for future research," Computers and Industrial Engineering, vol. 34, no. 1, pp. 3-20, 1998.

[11] G. Papaioannou and J. M. Wilson, "The evolution of cell formation problem methodologies based on recent studies (19972008): review and directions for future research," European Journal of Operational Research, vol. 206, no. 3, pp. 509-521, 2010.

[12] S. Kirkpatrick, J. Gelatt, and M. P. Vecchi, "Optimization by simulated annealing," Science, vol. 220, no. 4598, pp. 671-680, 1983.

[13] V. Černý, "Thermodynamical approach to the traveling salesman problem: an efficient simulation algorithm," Journal of Optimization Theory and Applications, vol. 45, no. 1, pp. 41-51, 1985.
[14] T.-H. Wu, C.-C. Chang, and S.-H. Chung, "A simulated annealing algorithm for manufacturing cell formation problems," Expert Systems with Applications, vol. 34, no. 3, pp. 1609-1617, 2008.

[15] F. Glover, "Future paths for integer programming and links to artificial intelligence," Computers and Operations Research, vol. 13, no. 5, pp. 533-549, 1986.

[16] S. Lozano, B. Adenso-Diaz, I. Eguia, and L. Onieva, "A one-step tabu search algorithm for manufacturing cell design," Journal of the Operational Research Society, vol. 50, no. 5, pp. 509-516, 1999.

[17] T.-H. Wu, C. Low, and W.-T. Wu, "A tabu search approach to the cell formation problem," The International Journal of Advanced Manufacturing Technology, vol. 23, no. 11-12, pp. 916-924, 2004.

[18] H. R. Lourenço, O. C. Martin, and T. Stützle, Iterated Local Search, Springer, 2003.

[19] H. R. Lourenço, O. C. Martin, and T. Stützle, "Iterated local search: framework and applications," in Handbook of Metaheuristics, pp. 363-397, Springer, 2010.

[20] P. Hansen, N. Mladenović, and J. A. M. Pérez, "Variable neighbourhood search: methods and applications," Annals of Operations Research, vol. 175, no. 1, pp. 367-407, 2010.

[21] A. Colorni, M. Dorigo, and V. Maniezzo, "Distributed optimization by ant colonies," in Proceedings of the First European Conference on Artificial Life (ECAL '91), vol. 142, pp. 134-142, Paris, France, 1991.

[22] X. Li, M. F. Baki, and Y. P. Aneja, "An ant colony optimization metaheuristic for machine-part cell formation problems," Computers \& Operations Research, vol. 37, no. 12, pp. 2071-2081, 2010.

[23] J. Kennedy, "Particle swarm optimization," in Encyclopedia of Machine Learning, pp. 760-766, Springer, 2010.

[24] Y. Shi and R. Eberhart, "A modified particle swarm optimizer," in Proceedings of the IEEE World Congress on Computational Intelligence, IEEE International Conference on Evolutionary Computation, pp. 69-73, May 1998.

[25] S.-C. Chu, P.-W. Tsai, and J.-S. Pan, "Cat swarm optimization," in PRICAI 2006: Trends in Artificial Intelligence, Q. Yang and G. Webb, Eds., vol. 4099 of Lecture Notes in Computer Science, pp. 854-858, Springer, 2006.

[26] P.-W. Tsai and V. Istanda, "Review on cat swarm optimization algorithms," in Proceedings of the 3rd International Conference on Consumer Electronics, Communications and Networks (CECNet '13), pp. 564-567, IEEE, Xianning, China, November 2013.

[27] E. Duman, M. Uysal, and A. F. Alkaya, "Migrating birds optimization: a new metaheuristic approach and its performance on quadratic assignment problem," Information Sciences, vol. 217, pp. 65-77, 2012.

[28] Q.-K. Pan and Y. Dong, "An improved migrating birds optimisation for a hybrid flowshop scheduling with total flowtime minimisation," Information Sciences, vol. 277, pp. 643-655, 2014.

[29] S. Niroomand, A. Hadi-Vencheh, R. Şahin, and B. Vizvári, "Modified migrating birds optimization algorithm for closed loop layout with exact distances in flexible manufacturing systems," Expert Systems with Applications, vol. 42, no. 19, pp. 6586-6597, 2015.

[30] R. Soto, B. Crawford, B. Almonacid, and F. Paredes, "A migrating birds optimization algorithm for machinepart cell formation problems," in Advances in Artificial Intelligence and Soft Computing, vol. 9413, pp. 270-281, Springer, Berlin, Germany, 2015. 
[31] I. Fister Jr., X.-S. Yang, I. Fister, J. Brest, and D. Fister, "A brief review of nature-inspired algorithms for optimization," Electrotechnical Review, vol. 80, no. 3, pp. 116-122, 2013.

[32] S. M. Almeida-Luz, M. M. Rodríguez-Hermoso, M. A. VegaRodríguez, J. Gómez-Pulido, and J. M. Sánchez-Pérez, "GRASP and grid computing to solve the location area problem," in Proceedings of the World Congress on Nature \& Biologically Inspired Computing (NaBIC '09), pp. 164-169, Coimbatore, India, December 2009.

[33] J.-H. Byun, K. Datta, A. Ravindran, A. Mukherjee, and B. Joshi, "Performance analysis of coarse-grained parallel genetic algorithms on the multi-core Sun UltraSPARC T1," in Proceedings of the IEEE (SOUTHEASTCON '09), pp. 301-306, IEEE, Atlanta, Ga, USA, March 2009.

[34] J. Homberger, "A parallel genetic algorithm for the multilevel unconstrained lot-sizing problem," INFORMS Journal on Computing, vol. 20, no. 1, pp. 124-132, 2008.

[35] C. Leon, G. Miranda, and C. Segura, "A memetic algorithm and a parallel hyperheuristic island-based model for a $2 \mathrm{D}$ packing problem," in Proceedings of the 11th Annual Genetic and Evolutionary Computation Conference (GECCO '09), pp. 13711378, ACM, July 2009.

[36] J. Jin, T. G. Crainic, and A. Løkketangen, "A cooperative parallel metaheuristic for the capacitated vehicle routing problem," Computers \& Operations Research, vol. 44, pp. 33-41, 2014.

[37] J.-M. Cutillas-Lozano and D. Giménez, "Optimizing sharedmemory hyperheuristics on top of parameterized metaheuristics," Procedia Computer Science, vol. 29, pp. 20-29, 2014.

[38] E. Alba, G. Luque, and S. Nesmachnow, "Parallel metaheuristics: recent advances and new trends," International Transactions in Operational Research, vol. 20, no. 1, pp. 1-48, 2013.

[39] M.-C. Albutiu, A. Kemper, and T. Neumann, "Massively parallel sort-merge joins in main memory multi core database systems," Proceedings of the VLDB Endowment, vol. 5, no. 10, pp. 10641075, 2012.

[40] A. Davidson, D. Tarjan, M. Garland, and J. D. Owens, "Efficient parallel merge sort for fixed and variable length keys," in Proceedings of the Innovative Parallel Computing (InPar '12), pp. 1-9, IEEE, San Jose, Calif, USA, May 2012.

[41] D. R. Helman, D. A. Bader, and J. Jájá, "A randomized parallel sorting algorithm with an experimental study," Journal of Parallel and Distributed Computing, vol. 52, no. 1, pp. 1-23, 1998.

[42] H. Peters, O. Schulz-Hildebrandt, and N. Luttenberger, "A novel sorting algorithm for many-core architectures based on adaptive bitonic sort," in Proceedings of the IEEE 26th International Parallel \& Distributed Processing Symposium (IPDPS '12), pp. 227-237, IEEE, 2012.

[43] I. S. Rajput, B. Kumar, and T. Singh, "Performance comparison of sequential quick sort and parallel quick sort algorithms," International Journal of Computer Applications, vol. 57, no. 9, pp. 14-22, 2012.

[44] V. Prifti, R. Bala, I. Tafa, D. Saatciu, and J. Fejzaj, "The time profit obtained by parallelization of quicksort algorithm used for numerical sorting," in Proceedings of the Science and Information Conference (SAI '15), pp. 897-901, London, UK, July 2015.

[45] P. Sanders and T. Hansch, "Efficient massively parallel quicksort," in Solving Irregularly Structured Problems in Parallel, vol. 1253 of Lecture Notes in Computer Science, pp. 13-24, Springer, Berlin, Germany, 1997.

[46] P. Tsigas and Y. Zhang, "A simple, fast parallel implementation of quicksort and its performance evaluation on sun enterprise
10000," in Proceedings of the 11th Euromicro Conference on Parallel, Distributed and Network-Based Processing, pp. 372-381, IEEE, Genova, Italy, Feburary 2003.

[47] E. Duman and I. Elikucuk, "Solving credit card fraud detection problem by the new metaheuristics migrating birds optimization," in Advances in Computational Intelligence, vol. 7903 of Lecture Notes in Computer Science, pp. 62-71, Springer, Berlin, Germany, 2013.

[48] K. Gao, P. Suganthan, and T. Chua, "An enhanced migrating birds optimization algorithm for no-wait ow shop scheduling problem," in Proceedings of the IEEE Symposium on Computational Intelligence in Scheduling (SCIS '13), pp. 9-13, IEEE, 2013.

[49] L. W. Shen, H. Asmuni, and F. C. Weng, "A modified migrating bird optimization for university course timetabling problem," Jurnal Teknologi, vol. 72, no. 1, 2014.

[50] P. B. S. Lissaman and C. A. Shollenberger, "Formation flight of birds," Science, vol. 168, no. 3934, pp. 1003-1005, 1970.

[51] J. P. Badgerow and F. R. Hainsworth, "Energy savings through formation flight? A re-examination of the vee formation," Journal of Theoretical Biology, vol. 93, no. 1, pp. 41-52, 1981.

[52] I. L. Bajec and F. H. Heppner, "Organized flight in birds," Animal Behaviour, vol. 78, no. 4, pp. 777-789, 2009.

[53] D. Hummel and M. Beukenberg, "Aerodynamische interferenzeffekte beim formationsflug von Vögeln," Journal of Ornithology, vol. 130, no. 1, pp. 15-24, 1989.

[54] J. Rayner, "A new approach to animal flight mechanics," Journal of Experimental Biology, vol. 80, no. 1, pp. 17-54, 1979.

[55] B. Almonacid, Dataset-Efficient Parallel Sorting for Migrating Birds Optimization when Solving Machine-Part Cell Formation Problems, Pontificia Universidad Católica de Valparaíso, Valparaíso, Chile, 2016, http://www.inf.ucv.cl/ balmonacid/ MPCFP/ParallelMBO2016. 

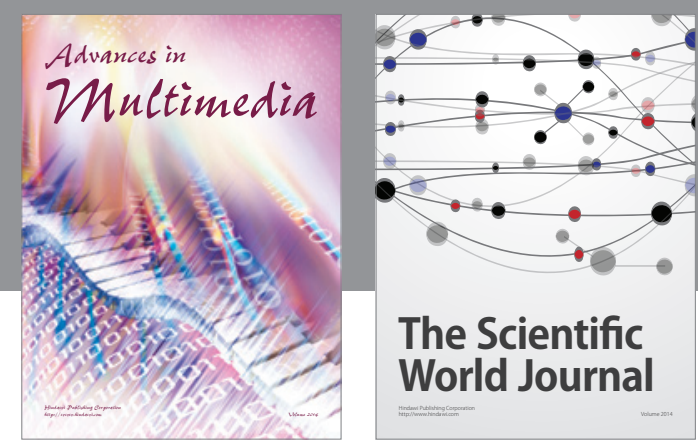

The Scientific World Journal
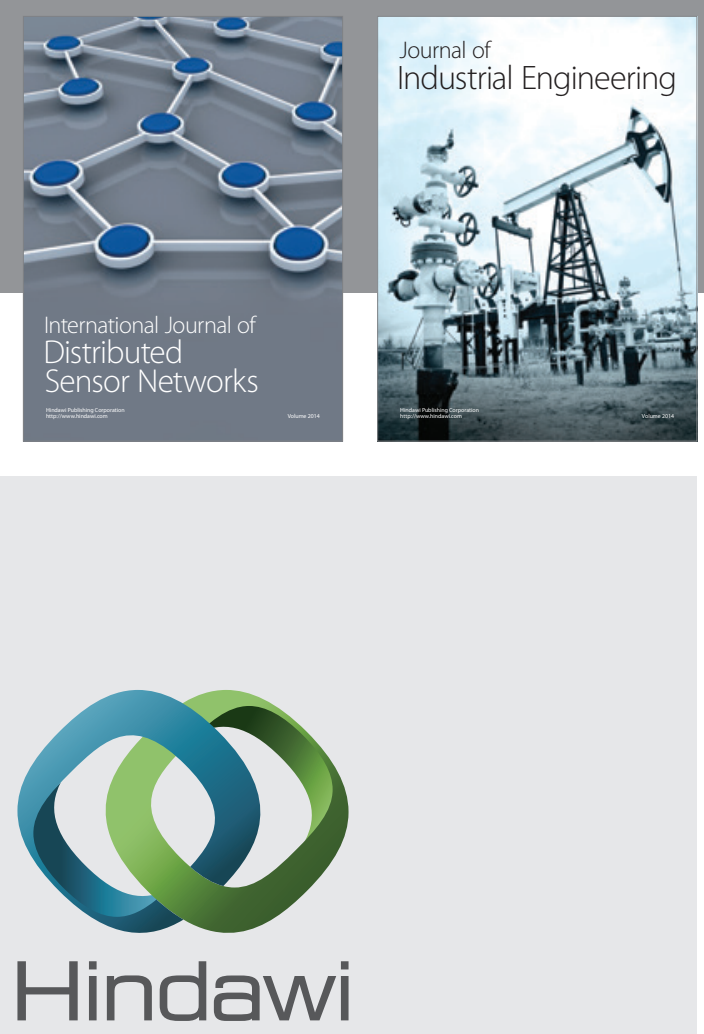

Submit your manuscripts at

http://www.hindawi.com

\section{Computer Networks} and Communications
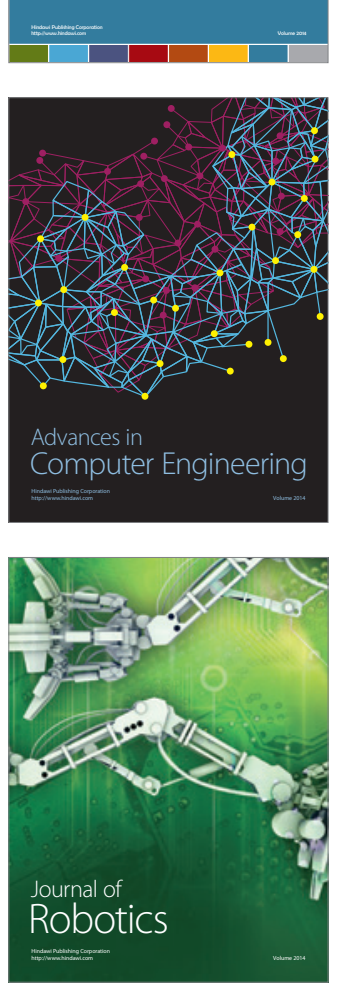
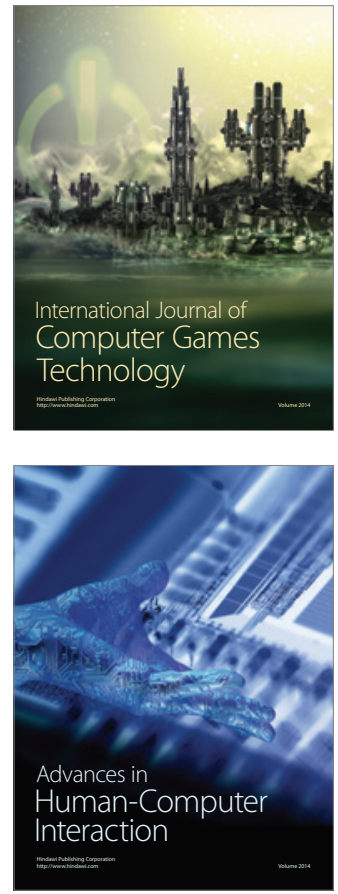
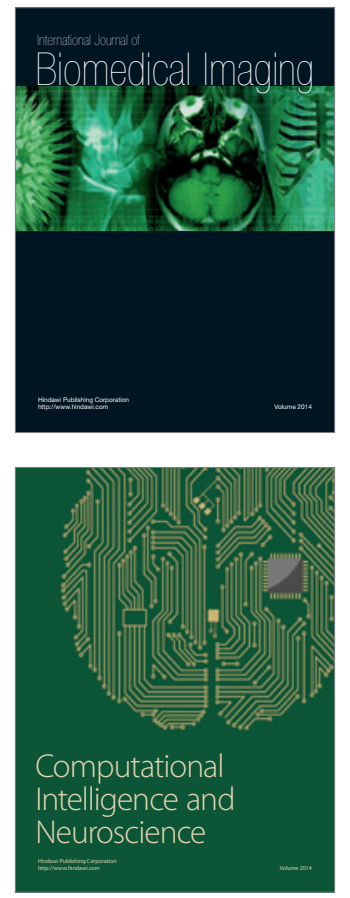
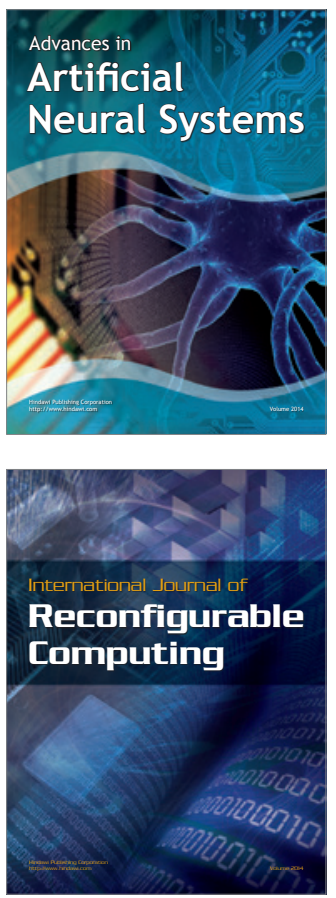
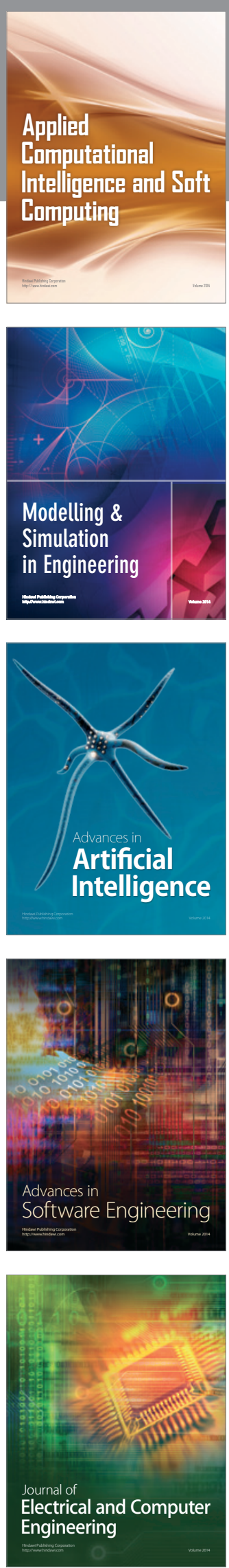